\title{
TOWARDS ROTATIONALLY STATE-RESOLVED DIFFERENTIAL CROSS SECTIONS FOR THE HYDROGEN EXCHANGE REACTION
}

\author{
Marcus Johannes Jacobus Vrakking
}

Chemical Sciences Division, Lawrence Berkeley Laboratory, and Department of Chemistry, University of Califomia at Berkeley, Berkeley, California 94720, USA

November 1992

This work was supported by the Director, Office of Energy Research, Office of Basic Energy Sciences, Chemical Sciences Division, of the U.S. Department of Energy under Contract No. DE-AC03-76SF00098. 
Towards rotationally state-resolved differential cross sections for the hydrogen exchange reaction

by

Marcus Johannes Jacobus Vrakking

\begin{abstract}
The hydrogen exchange reaction $\mathrm{H}+\mathrm{H}_{2} \rightarrow \mathrm{H}_{2}+\mathrm{H}$ (and its isotopic variants) plays a pivotal role in chemical reaction dynamics. It is the only chemical reaction for which fully converged quantum scattering calculations have been carried out using a potential energy surface which is considered to be chemically accurate. To improve our ability to test the theory, a 'perfect experiment', measuring differential cross sections with complete specification of the reactant and product states, is called for.

In this thesis, the design of an experiment is described that aims at achieving this goal for the $D+\mathrm{H}_{2}$ reaction. A crossed molecular beam arrangement is used, in which a photolytic $D$ atom beam is crossed by a pulsed beam of $\mathrm{H}_{2}$ molecules. DH molecules formed in the $\mathrm{D}+\mathrm{H}_{2}$ reaction are state-specifically ionized using Dopplerfree $(2+1)$ Resonance-Enhanced Multi-Photon Ionization (REMPI) and detected using a position-sensitive microchannel plate detector. This detection technique has an unprecedented single shot detection sensitivity of $6.810^{3} \mathrm{molecules} / \mathrm{cc}$. This thesis does not contain experimental results for the $\mathrm{D}+\mathrm{H}_{2}$ reaction yet, but progress that has been made towards achieving this goal is reported.

In addition, results are reported for a study of the Rydberg spectroscopy of the
\end{abstract}


water molecule. One- and two-photon ionization of the water molecule are reported, showing rotationally resolved lines corresponding to transitions to members of Rydberg series (nd $<-1 b_{1} ; n=\geq 6$ ) converging on the $\mathrm{H}_{2} \mathrm{O}^{+}(100)$ and $\mathrm{H}_{2} \mathrm{O}^{+}(000)$ vibrational states, respectively. The results are simulated using multichannel quantum defect theory (MQDT) and it is argued that discrepancies between experiment and theory arise from perturbative interactions between the (nd $<--1 b_{1}$ ) levels and members of (nd <-- $3 a_{1}$ ) Rydberg series, as well as selective predissociation. 


\section{CONTENTS}

Introduction

Acknowledgements viii

Chapter I: Resonance-enhanced one- and two-photon ionization of the water molecule: preliminary analysis by quantum defect theory 1

Abstract 2

1. Introduction 3

An Introduction of multi-channel quantum defect theory 4

$\mathrm{H}_{2} \mathrm{O}$ Rydberg spectroscopy 9

2. Experimental approach

3. Results and analysis 16

Quantum defect analysis $\quad 26$

4. Concluding remarks 30

References $\quad 32$

Tables 34

Chapter II: Ultra-sensitive detection of hydrogen molecules by $(2+1)$ REMPI 45

Abstract $\quad 46$

1. Introduction 47

2. Theory 48

Perturbation theory of one- and two-photon transitions 49

Comparison of the detection efficiencies for molecular hydrogen using (1+1) REMPI and (2+1) REMPI 54

3. Experimental 59

4. Results 63

The power dependence of $(2+1)$ REMPI of $\mathrm{H}_{2}(\mathrm{~J}=1)$

The ionization efficiency of $\mathrm{DH}(\mathrm{J}=0)$ by $(2+1)$ REMPI 66

$\begin{array}{ll}\text { 5. Discussion } & 69\end{array}$

$\begin{array}{ll}\text { References } & 72\end{array}$ 
Chapter III: Design of a new crossed molecular beam experiment to measure fully state-resolved angular distributions for the hydrogen exchange reaction

Abstract

1. Introduction

2. Description and feasibility analysis of the proposed technique to measure fully state-resolved angular distributions

2.1 The basis principle and the experimental set-up 81

2.2 Yield of reactive product for $\mathrm{D}+\mathrm{H}_{2}-->\mathrm{DH}+\mathrm{H} \quad 99$

D atom production $\quad 100$

$D$ atom transport to the interaction region and calculation of reactive product

2.3 Laser detection of reaction products from $\mathrm{D}+\mathrm{H}_{2}->$ $\mathrm{DH}+\mathrm{H}$

3. Conclusions 113

References

Chapter IV: Experimental progress in studies of fully state-resolved differential cross sections for the $\mathrm{D}+\mathrm{H}_{2}$ reaction

Abstract

1. Introduction

2.1 Diagnostics for the $D$ atom beam

2.2 Diagnostics for the $\mathrm{H}_{2}$ beam

2.3 Attenuation of the $D$ atom beam

2.4 Molecular hydrogen in the $D$ atom beam

2.5 The first observation of possible signal from $\mathrm{D}+\mathrm{H}_{2}$ and outlook for the future

Appendix A: The $\mathrm{I}^{\circ}$ ratio in the photodissociation dynamics of DI at $205 \mathrm{~nm}$

References 


\section{INTRODUCTION}

In the preparation of this thesis, one of the more difficult tasks was to come up with a title. The most logical choice would be something like "Fun with lasers and molecular beams", but titles in this style are not that common. A title like this would have been somewhat appropriate however, since, during my time here at Berkeley, we have been involved in a rather wide variety of experiments, ranging from ultra-high resolution spectroscopy of the $\mathrm{Kr}$ atom ${ }^{1)}$, to photodissociation dynamics of molecules like cyclohexadiene $^{2)}$ and ethylene ${ }^{3)}$; from Rydberg spectroscopy of the water molecule ${ }^{4)}$, in the extreme ultraviolet part of the wavelength spectrum, to overtone spectroscopy of the benzene molecule ${ }^{\text {) }}$ in the infrared; and ultimately, crossed molecular beam studies of the hydrogen exchange reaction ${ }^{6}$, with along the way experiments on two-photon excitation of molecules like hydrogen ${ }^{7}$ and nitrogen ${ }^{8}$. A variety of different experiments with no single underlying theme, except perhaps the consistent use of high power near transform-limited tunable lasers and molecular beam techniques.

In this sense the experiments listed above, some of which are discussed in this thesis, illustrate the many ways in which lasers and molecular beams have revolutionized the field of physical chemistry in the last twenty years. The virtues of the use of molecular beams have been well documented"). First of all, the use of molecular beams has enabled physical chemists to study chemical reactions under single-collision conditions with well-defined initial conditions. In the literature there are numerous examples of experiments where final product state distributions, which are an important signature of the dynamics of a chemical process, are affected by collisional relaxation of the reaction products. The use of molecular beam techniques is a convenient way to avoid this problem. On the other hand, in spectroscopy experiments, the internal state cooling in a supersonic expansion allows for simplified assignments. An example is the photoionization of the water molecule, which is discussed in Chapter I of this thesis. The rotational cooling achieved in a molecular beam provides a spectrum which is sufficiently sparse that assignments can be made with some confidence. Another interesting example is the overtone spectroscopy of the 
benzene molecule, an area where our group has been active in the past ${ }^{5.10)}$, and where more experiments are planned in the future. The objective here is to study the rate of intra-molecular vibrational relaxation (IVR), by measuring the linewidths of successive $\mathrm{C}-\mathrm{H}$ overtones. In conventional gas cell absorption spectroscopy, very wide absorption bands are seen ${ }^{11)}$, and it is not clear whether the width is homogeneous or heterogeneous. The use of a double-resonance molecular beam technique can give definitive answers. Finally, an additional advantage of the use of molecular beams is the fact that molecular beams operate in high vacuum, where, with the exception of photon detection techniques, it is easier to use sensitive detectors capable of detecting single chemical events.

The contributions that lasers have made to the field of physical chemistry are no less impressive. Lasers are widely used in the preparation of reactants, the inititation of chemical processes and the state-specific detection of reaction products. The use of tunable lasers allows one to prepare atoms and molecules in well-defined internal states, enabling experiments which explore the role of vibrational or electronic excitation on reaction cross sections. Interesting examples are the reactions of electronically excited alkali and alkaline earth atoms studied in our group ${ }^{12}$, and studies of the hydrogen exchange reaction with vibrationally excited reactants, in the Zare group at Stanford ${ }^{13}$. Crossed molecular beams experiments have become possible with radical beams, that are produced by laser photolysis of a suitable precursor molecule ${ }^{14)}$, and the study of unimolecular decomposition following laser excitation has grown into one of the most important and rewarding endeavours in physical chemistry ${ }^{15)}$. Perhaps most important of all however, is the way in which lasers have enabled state-specific detection of the products of chemical processes. Detection techniques such as Resonance-Enhanced Multi-Photon Ionization (REMPI), LaserInduced Fluorescence (LIF), Coherent Anti-Stokes Raman Scattering (CARS), and, recently, Degenerate Four-Wave Mixing, provide state-specific detection schemes for an ever-increasing number of molecules and radicals with very high sensitivities, allowing the dynamics to be probed in unprecedented detail. An example of the sensitivity of some of these schemes is given in Chapter II, where the state-specific 
detection of hydrogen molecules is reported, using Doppler-free $(2+1)$ REMPI. While hydrogen is one of the most difficult molecules to electronically excite or ionize, a detection sensitivity is found where a density of only $10^{4}$ molecules per cubic centimeter in a given ro-vibrational state is sufficient for a detectable signal. Finally, in the last few years, the use of ultrafast lasers has added an additional dimension to experimental work, where, for the first time, it has now become possible to study a large number of chemical processes such as photodissociation, isomerization and intramolecular vibration relaxation in real time ${ }^{16)}$.

To the extent that the hydrogen exchange reaction is often regarded as the benchmark system that most progress in theoretical chemistry has evolved around, it is not inappropriate to assign this chemical system a similar status in the field of experimental physical chemistry. And as such, the progress in the last twenty years in experimental studies of the hydrogen exchange reaction, is the story of the application of molecular beam techniques and laser techniques to experiments on this reaction ${ }^{17}$. Using molecular beam techniques, experiments have evolved from measurements of rate constants to measurements of differential cross sections. And, using laser techniques, measurements of product state distributions have become possible, as well as greatly improved crossed molecular beam experiments with photolytic hydrogen atom beams. Our most ambitious project in the last few years has been an attempt to combine all these new technologies into one experiment. This experiment, which is still in progress at the completion of this thesis, is a crossed molecular beam experiment, in which a photolytically generated D atom beam is crossed with a pulsed beam of $\mathrm{H}_{2}$. Product $\mathrm{DH}(\mathrm{v}, \mathrm{j})$ molecules are state-specifically ionized by Doppler-free $(2+1)$ REMPI and detected using a position-sensitive microchannel plate detector. The design of this unique experiment is described in detail in Chapter III, and the experimental progress that has been made sofar, is described in Chapter IV. 


\section{REFERENCES}

1) T. Trickl, M.J.J. Vrakking, E. Cromwell, Y.T. Lee and A.H. Kung, Phys. Rev. A $\underline{39}, 2948$ (1989).

2) E.F. Cromweil, D.-J. Liu, M.J.J. Vrakking, A.H. Kung and Y.T. Lee, J. Chem. Phys. 92, 3230 (1990); ibid 95, 297 (1991).

3) E.F. Cromwell, A. Stolow, M.J.J. Vrakking and Y.T. Lee, J. Chem. Phys. 97, 4029 (1992).

4) M.J.J. Vrakking, Y.T. Lee, R.D. Gilbert and M.S. Child, J. Chem. Phys. (accepted).

5) D.-J. Liu, A. Stolow, M.J.J. Vrakking, E.F. Cromwell and Y.T. Lee, to be published.

6) M.J.J. Vrakking, A.S. Bracker and Y.T. Lee, Book of Abstracts, XIIIth International Symposium on Molecular Beams, El Escorial, Madrid, Spain, 2-7 june 1991.

7) M.J.J. Vrakking, A.S. Bracker, T. Suzuki and Y.T. Lee, Rev. Sci. Instrum. (accepted).

8) M.J.J. Vrakking, A.S. Bracker and Y.T. Lee, J. Chem. Phys. 96, 7195 (1992).

9) G. Scoles in "Atomic and Molecular Beam Methods", Vol. I, edited by G. Scoles, Oxford University Press (1988), p. 3.

10) R.H. Page, Y.R. Shen and Y.T. Lee, J. Chem. Phys. 요, 4621 (1988).

11) R.G. Bray and M.J. Berry, J. Chem. Phys. 71, 4909 (1979).

12) H.F. Davis, A.G. Suits and Y.T. Lee, in "Gas Phase Metal Reactions", edited by A. Fontijn, Elsevier (1992).

13) D.A.V. Kliner and R.N. Zare, J. Chem. Phys. 92, 2107 (1990).

14) S.A. Buntin, C.F. Giese and W.R. Gentry, J. Chem. Phys. 87, 1443 (1987).

15) See "Advances in Gas-Phase Photochemistry and Kinetics - Molecular Photodissociation Dynamics", edited by M.N.R. Ashfold and J.E. Baggott (1987).

16) M.J. Rosker, M. Dantus and A.H. Zewail, J. Chem. Phys. $\underline{89}, 6113$ (1988); 
ibid. $\underline{89}, 6128$ (1988).

17) See J.J. Valentini and D.L. Phillips in "Advances in Gas-Phase Photochemistry and Kinetics - Bimolecular Collisions", edited by M.N.R. Ashfold and J.E. Baggott, (1989), p. 1-51. 


\section{ACKNOWLEDGEMENTS}

Over the last few years a large number of people have contributed to the success of the work described in this thesis. First and foremost among these has been Yuan Lee as my research director. Yuan's sincere dedication to science has been well documented by the many students who have obtained their PhD's in his group. I have particularly enjoyed the almost limitless freedom that Yuan gives the people in his group to pursue their personal interests. I am sure a lot of former Lee-group members will agree when I say that there is something a little magical that happens in all these scientific discussions in Yuan's office, where you find yourself walking out afterwards with just that little bit of extra enthusiasm, and just that little bit of extra confidence that the experiment is going to work. It is what makes the difference between just doing interesting research and wanting to do what have respectfully come to be known as "Lee group experiments". After the completion of this thesis, I very much look forward to continuing my work in Yuan's group for some time, although it will never be long enough to pursue all the interesting ideas that have come up and keep coming up.

There have been a large number of co-workers whom I have had the pleasure of working with over the past few years. The first of these co-workers was Tom Trickl, who got me started on the vacuum-ultraviolet machine and with whom I cooperated on the $\mathrm{Kr}$ and hydrogen dimer experiment. Tom was an excellent teacher and, with the development of the "Trickl valve", he has certainly left a lasting mark on the group.

My longest cooperation was with Evan Cromwell, who introduced me to lasers and, perhaps more importantly, the Califomian way of life. As I recall, it was not always easy to make it back to the laboratory after enjoying the early morning cappucino's at Cafe Strada, but during the non-frisbee semesters we did actually get some nice experiments done. It is fun to remember those scientific discussions where Evan would just stop in mid-sentence when an attractive girl would pass by.

I thoroughly enjoyed working with Albert Stolow during the time he spent in 
our lab. Whether the subject was science, (American) politics or art, discussions with Albert were always interesting and in the lab there was never a dull moment, because, let's face it, the man likes to talk! I am very much indebted to Albert for some crucial suggestions that he made in the development of the $\mathrm{D}+\mathrm{H}_{2}$ experiment, although, in all honesty, I probably appreciated the way he introduced me to blues music even more. I wish him all the best in his new job at NRC.

My two co-workers on the $\mathrm{D}+\mathrm{H}_{2}$ experiment were Toshi Suzuki and Allan Bracker. Toshi brought in some helpful experience concerning the use of microchannel plates and was a nice discussion partner on subject matters ranging from the wonderful $\mathrm{D}+\mathrm{H}_{2}$ data that, no doubt, we were going to see very soon now, to the intricacies of Japanese society and the voice of Carol Vaness. Allan has developed into a tireless contributor in the lah, and I am especially indebted to him for all the computer softwart that he prosared for our experiments. Don't worry, it's going to happen any day now, Alian!

A number af current or former Lee group members deserve special mention for their contributions. I much enjoyed working with Peter Weber on the early development of a photoelectron spectrometer and I am glad to hear he is now successfully continuing this work at Brown. Bob Continetti provided me with a lot of useful experience from his $\mathrm{D}+\mathrm{H}_{2}$ experiment and taught many of us a little something about the art of international diplomacy at scientific conferences. Deon Anex provided a rational viewpoint in animated scientific discussions and was always willing to lend a hand in the lab. I am most indebted to him, however, for his suggestions on where to go in New Orleans. Mark Crofton and I became very good friends during his time in Berkeley. Mark and I spent many hours discussing the many facets of life in the States, and I fondly remember our trip to Baja last year. OK, so we didn't quite see the solar eclipse, but they sure served a good margherita down there. Floyd Davis and Arthur Suits set a standard in the group for enthusiasm and dedication, that was very inspiring. Also, they invariably were ideal candidates when it came to picking a cultural fight. James Chesko did and does an enthusiastic job keeping the computer facilities in the group operational. Sure, most of the time you don't know what he is 
trying to fix, and sometimes it does not work at the first try, but in the end he usually comes through. On many occasions it was useful to consult Andy Kung on laser related matters. Strada-discussions with Laurent Nahon were always fun and sometimes enlighten: $s$.

And then there is perhaps the most important Lee group member of all, our group secretary Ann Lawhead. Ann was always very helpful getting our paperwork through the system in a hurry, and as the unofficial 'mother of the group' always provided a willing ear for the occasional frustrations. Most important however, if you ever need to know what is going on in the department or at LBL...

During my time at Berkeley I developed a little bit of an appetite for setting up new experiments, and a great number of people were instrumental in tuming the paper dreams into reality. George Weber provided invaluable advice on the do's and don'ts of building a big vacuum chamber - there's no doubt his move to physics was a loss for the department. Hans Graetsch and Chuck Souders did the bulk of the machining that went into building the $\mathrm{D}+\mathrm{H}_{2}$ vacuum chambers and our new vacuum-ultraviolet chamber, doing a wonderful job throughout. Scott Bonilla welded it all together and Eric Granlund did a very nice job building what is probably one of the world's smaller gate valves.

Some people outside Berkeley contributed in important ways as well. I very much enjoyed working with professor Mark Child from Oxford University on the photoionization of the water molecule and am very much indebted to him for first encouraging us to do the experiment, and then performing the quantum defect calculations reported in Chapter I. I greatly enjoyed visiting him in Oxford, where I learned a lot about quantum defect theory, but perhaps even more about Oxford history.

On the $\mathrm{D}+\mathrm{H}_{2}$ experiment I have benefitted enormously from a cooperation with Dr. Koichiro Oba and Motohiro Suyama from Hamamatsu Photonics. Their offer to build a position-sensitive ion detector for us was a small gift from heaven, and throughout the experiments, when the going sometimes got a little tougher than anticipated, they have gone out of their way to keep us going with the shortest 
possible time delays. Many arigato's!

Two people deserve special mention for the influence they had before I came to Berkeley. Dr. Herman Beyerinck from the Eindhoven University of Technology (Netherlands) gave me my first start in science, and provided me with an excellent role model. It was he who wetted my appetite for foreign travel and arranged my stay in the laboratory of Dr. Kosuke Shobatake, at the Institute for Molecular Science (Japan). "Shobatake-san" introduced me to crossed molecular beam experiments, and I fondly remember the countless nights when, before going hor he would stop by to see how things were going, and ended up working alongside till deep in the night. It was those nights filled with recollections from his time here in Berkeley, that set me on my way to working for Yuan. 


\section{CHAPTER I}

RESONANCE-ENHANCED ONE- AND TWO-PHOTON IONIZATION OF THE WATER MOLECULE:

PRELIMINARY ANALYSIS BY

MULTICHANNEL QUANTUM DEFECT THEORY 


\begin{abstract}
Experimental results are presented for one- and two-photon ionization of the water molecule, obtained using a near transform-limited extreme ultraviolet (xuv) laser. The single-photon ionization results show rotationally resolved auto-ionizing resonances corresponding to members of Rydberg series (nd <-- $1 b_{1} ; n=6-11$ ) converging on the $\mathrm{H}_{2} \mathrm{O}^{+}(100)$ vibrational state. The $(1+1)$ multi-photon ionization results show rotationally resolved structure corresponding to Rydberg series (nd <-- $1 b_{1} ; n \geq 6$ ) converging on the $\mathrm{H}_{2} \mathrm{O}^{+}(000)$ vibrational state. Typical linewidths below and above the $\mathrm{H}_{2} \mathrm{O}^{+}(000)$ ionization threshold are $1 \mathrm{~cm}^{-1}$ and $2 \mathrm{~cm}^{-1}$, respectively.

The experimental results are simulated by multichannel quantum defect theory (MQDT). rain features in the spectrum are reproduced in a treatment of the rotational channel interactions with partial $l$ mixing. It is argued that remaining discrepancies between experiment and theory arise from perturbative interactions between the (nd <-- $1 b_{1}$ ) levels and members of the (nd <-- $3 a_{1}$ ) Rydberg series. Also, it is argued that in the $(1+1)$ multi-photon ionization spectra lines may be missing due to selective predissociation.
\end{abstract}




\section{Introduction}

In recent years, the photoionization spectrum of the water molecule has become the model system of choice for the treatment of the Rydberg spectra of a polyatomic molecule by multichannel quantum defect theory (MQDT). Photoionization spectra obtained by Page et. al." first showed a number of auto-ionizing Rydberg series converging on the (100) vibrational state of the $\mathrm{H}_{2} \mathrm{O}^{+}$ion in its ground electronic state, and this provided the impetus for Child and Jungen to carry out the first MQDT calculation for an asymmetric top molecule ${ }^{2)}$.

The results of Page et. al. showed resolved rotational structure for principal quantum number $n=6$, which is the lowest member of these Rydberg series above the (000) ionization threshold, but for higher members of the Rydberg series only the very strongest rotational features could be observed. Recent improvements in the generation of extreme ultra-violet radiation (xuv) have enabled an experiment where the rotational structure of the $\mathrm{H}_{2} \mathrm{O}$ Rydberg series can be followed to higher principal quantum number, with the additional advantage of improved resolution. These new spectra are presented in this chapter.

In addition, results are presented for an experiment where $\mathrm{H}_{2} \mathrm{O}$ Rydberg series converging on the (000) vibrational state of the $\mathrm{H}_{2} \mathrm{O}^{+}$ion were studied by employing a $(1+1)$ multi-photon ionization (MPI) technique. These Rydberg series are expected to have quantum defects very similar to the series observed in the direct photoionization experiment, and thus their observation aids in the assignments of the photoionization spectra. Furthermore, differences between these MPI spectra and the photoionization results give information about the decay mechanisms of the Rydberg states and the extent to which these states are perturbed by neighbouring states.

Efforts were made to simulate the spectra using multichannel quantum defect theory ${ }^{3-5)}$, with a description in terms of rotational channel interactions with partial $l$ mixing. Although the simulations were able to reproduce some of the main features of the spectra, some serious discrepancies remain. It is believed that these discrepancies are due to perturbations by overlapping excitations to linear ionic states. 
An Introduction to Multi-Channel Quantum Defect Theory

In conventional treatments of a molecular spectroscopy problem, the approach that is usually taken is to separate the molecular problem into an electronic problem on one hand, and a ro-vibrational nuclear problem on the other, by application of the BornOppenheimer approximation. In this approximation it is assumed that the electrons in the molecule move much faster than the nuclei, so the nuclear motion can be calculated by considering the mutual nuclear interactions and the interactions of the nuclei with a time-averaged field set up by the electrons. A practical approach consists of solving the electronic problem for a number of possible nuclear geometries. This defines the different potential energy surfaces for the nuclear motion and shows how the electron motion is coupled to the molecular frame. If the potential energy surface is known a ro-vibrational analysis of the molecular energy level structure can readily be carried out, and thus the spectrum of possible molecular transitions is obtained.

When the molecular spectroscopy problem involves excitations to high Rydberg orbitals, the conventional techniques break down, since the necessary separation of time-scales for the nuclear and electronic motion, which was the key to the BornOppenheimer approximation, no longer exists. For electrons in sufficiently high Rydberg orbitals, except during brief intervals when the Rydberg electron moves close to the ionic core of the molecule, the velocity of the Rydberg electron is slower than the velocities of the nuclei, and the electron motion, not being able to keep up with the motion of the ionic molecular core, is no longer coupled to the molecular frame. Rather than in the molecular frame, the orbital angular momentum of the electrum has well-defined projections in the laboratory frame. This case of $l$-uncoupling is described by the transition from Hund's case (b) to Hund's case (d).

While the energy levels for the ionic molecular core can be determined within the normal Born-Oppenheimer approximation, a calculation of the energy levels of the neutral molecule requires an adequate description of the interaction of the Rydberg electron with the ionic core. A possible approach would be the use of scattering theory, where a description would be given in terms of inelastic collisions of the 
electron with the ionic core with the possibility of transitions between different corestates, however this approach is impractical due to the complex nature and strong energy dependence of the S-matrix. The use of MQDT is based on recognizing first of all the fact that the collision process occurs after the Rydberg electron has been accelerated under the Coulomb interaction with the core, and therefore the collision energy depends only very weakly on the exact initial energy of the electron. In other words, the collision proces is almost independent of the exact initial state of the electron and does not, for example, depend very strongly on its bound or free nature at large radial distances. The interaction can be represented by a scattering phase shift which only depends on the way in which the electron couples to the molecular frame at short range. The quantum defect $\mu$ that gives MQDT its name, is this scattering phase shift divided by $\pi$, and will be shown to determine the energy structure of the Rydberg states.

The second key to MQDT is that at long range the electron interacts with the core via essentially only an isotropic Coulomb interaction, and in this range solutions for the radial electron wave function, which are called Coulomb functions, can be obtained analytically. At every energy there are two independent Coulomb functions which by themselves do not obey regular asymptotic boundary conditions.

$\mathrm{MQDT}^{3-5)}$ is based on writing a general expression for the asymptotic electron wavefunction in terms of Coulomb functions and in terms of the quantum defects that follow from the short-range interaction. In writing down this wave function, a central role is played by the frame-transformation that relates the angular wave functions which apply at small electron-core distances, where the electron motion is coupled to the molecular frame (Hund's case (b)), and at large distances, where the electron is no longer coupled to the molecular frame and the angular wavefunction is better described in Hund's case (d). The total electron wave function is general in a sense that it is a superposition of functions corresponding to different configurations where the angular dependence is described in Hund's case (b) or (d). Following from the asymptotic behaviour of the radial Coulomb wave functions, the functions in the superposition by themselves do not satisfy regular asymptotic boundary conditions, and 
physically relevant solutions are obtained by determining the superpositions which do satisfy these boundary conditions. This procedures, which is referred to as the elimination of closed channels, gives the energy levels of the Rydberg states.

The first application of quantum defect theory to a molecular problem was given by Fano in 1970 and presented a discussion of the photoionization of the hydrogen molecule starting from its ground ro-vibrational state, following excitation to a high np Rydberg orbital ${ }^{4)}$. In order to make some of the concepts which were described above a little clearer, some of Fano's calculations will be shown here. As discussed above, the molecule is appropriately described in Hund's case (b) or Hund's case (d), depending on the velocity of the Rydberg electron. When the Rydberg electron is close to the core, the Born-Oppenheimer approximation applies and the molecule is fully characterized by stating whether the projection of the electron orbital angular momentum $(l=1)$ on the internuclear axis equals $0(\sigma)$ or $1(\pi)$. At long range the electron orbital angular momentum is decoupled from the internuclear axis, and the molecule can be characterized by stating whether the total angular momentum $\mathrm{J}=1$ is obtained by combining the electron orbital angular momentum $l=1$ with the nuclear rotational angular momentum $\mathrm{N}=0$ or $\mathrm{N}=2$. The frame transformation described above, thus relates the angular wavefunctions for the $\sigma$ and $\pi$ states to those for the $N=0$ and 2 states, and can be derived analytically. The short-range interaction is described by two quantum defects, labelled $\mu_{\sigma}$ and $\mu_{x}$.

The general expression for the asymptotic electron wavefunction is given as

$$
\begin{aligned}
\Psi\left(\overrightarrow{R_{,},}, r\right) & =\sum_{\Lambda=0,1} \sum_{N=0,2} \Phi^{N}(r, R) * \\
& {\left[f\left(v_{N} r\right) U_{N \Lambda} \cos \pi \mu_{\Lambda}-g\left(v_{N} r\right) U_{N \Lambda} \cos \pi \mu \Lambda\right] A_{\Lambda} }
\end{aligned}
$$


where $v_{N}$ is the effective quantum number, which is defined by

$$
e_{N}-e_{N}^{+}-\frac{R y}{v_{N}^{2}}
$$

and $f$ and $g$ are the Coulomb wavefunctions. For Rydberg orbitals where the distance from the electron to the core is small, the effective quantum number $v_{\mathrm{N}} \approx V$ and $\Psi$ reduces to a sum over $\Phi^{\wedge}$, which is the appropriate description in this range. On the other hand, Equation (1) is also a summation over $\Phi^{\mathrm{N}}$, which is the appropriate description for large electron-core distances. The above-mentioned elimination of closed channels is performed by choosing appropriate coefficients $A_{\Lambda}$. In order to illustrate this by an example, an energy range below the threshold for formation of $\mathrm{H}_{2}{ }^{+}(\mathrm{N}=0)$ will now be chosen, where both the $\mathrm{N}=0$ and the $\mathrm{N}=2$ channel are closed, and it will be seen how the Rydberg structure in this energy range can be determined. Using the known analytical forms of the Coulomb functions ${ }^{3)}$, the following expression can be written down for the total asymptotic wavefunction of the Rydberg electron,

$$
\begin{aligned}
\Psi= & \Phi^{0}\left[u\left(v_{0}, r\right) \sum_{\Lambda} U_{Q \Lambda} \sin \pi\left(v_{0}+\mu_{\Lambda}\right) A_{\Lambda}-v\left(v_{0}, r\right) \sum_{\Lambda} U_{Q \Lambda} \cos \pi\left(v_{0}+\mu_{\Lambda}\right) A_{\Lambda}\right]+ \\
& \Phi^{2}\left[u\left(v_{2}, r\right) \sum_{\Lambda} U_{2 \Lambda} \sin \pi\left(v_{2}+\mu_{\Lambda}\right) A_{\Lambda}-v\left(v_{2}, r\right) \sum_{\Lambda} U_{2 \Lambda} \cos \pi\left(v_{2}+\mu_{\Lambda}\right) A_{\Lambda}\right]
\end{aligned}
$$

where $u(v, r)$ is an exponentially diverging function of $r$ and $v(v, r)$ is an exponentially decaying function of $r$. The elimination of the closed channels $\mathrm{N}=0$ and $\mathrm{N}=2$ means that coefficients $A_{\Lambda}$ need to be chosen so that the coefficients of $u\left(v_{i}, r\right)$ are zero. It can readily be seen how in a single channel situation this would lead to the requirement that $v+\mu$ be integer, which leads to the well-known Rydberg formula with the energy expressed in terms of an effective quantum number $v$, which deviates from an integer by the quantum defect $\mu$. In the multi-channel case the situation is a little more complex however, since, as can be seen from Equation (3), the equations relating the effective quantum numbers in the asymptotic channels corresponding to $\mathrm{N}=0$ and $\mathrm{N}=2$ to the quantum defects $\mu_{\sigma}$ and $\mu_{x}$, are coupled. For the two-channel case discussed 
here, a non-trivial solution of the coefficients $A_{\Lambda}$ can be obtained if the determinant

$$
\left[\begin{array}{cc}
\cos \alpha \sin \pi\left(v_{0}+\mu_{0}\right) & \sin \alpha \sin \pi\left(v_{0}+\mu_{\pi}\right) \\
-\sin \alpha \sin \pi\left(v_{2}+\mu_{0}\right) & \cos \alpha \sin \pi\left(v_{2}+\mu_{\pi}\right)
\end{array}\right]
$$

is zero, where $\cos \alpha=\mathrm{U}_{0 \sigma}=\mathrm{U}_{2 \pi}$, and $\sin \alpha=\mathrm{U}_{0 \pi}=-\mathrm{U}_{2 \sigma}$. This defines a relation between the two effective quantum numbers $v_{0}$ and $v_{2}$ in the asymptotic channels corresponding to $\mathrm{N}=0$ and $\mathrm{N}=2$, which can be represented in a so-called Lu-Fano plot. Together with the energy-equation

$$
e-e_{0}^{+}-\frac{R y}{v_{0}^{2}}=\varepsilon_{0}^{+}+6 B-\frac{R y}{v_{2}^{2}}
$$

which defines the effective quantum numbers, this leads to two equations for the unknown effective quantum numbers $v_{0}$ and $v_{2}$, and thus the effective quantum numbers and the energies of the Rydberg states can be calculated. This two-channel quantum defect calculation beautifully reproduces the experimental absorption spectrum, which shows evidence of substantial perturbations between the two asymptotic channels ${ }^{4.5}$. For more details the reader is referred to Fano's original paper $^{4)}$ and the excellent review by Greene and Jungens).

In the case of $\mathrm{H}_{2} \mathrm{O}$ photoionization, the main difference with the treatment described above is the complexity of the frame-transformation from Hund's case (b) to Hund's case (d) for an asymmetric top molecule. This transformation was worked out in detail by Child and Jungen ${ }^{2)}$, and applies the following description of the molecule. At close range the Rydberg electron is coupled to the molecular ion core and the projection $\lambda$ of the electron orbital angular momentum $l$ is defined. The rotational state of an asymmetric top molecule is normally specified by the total angular momentum $\mathbf{J}$ with its projection $\mathrm{M}$ in the laboratory frame, and its projections in the molecular frame are approached as $K_{c}$ and $K_{c}$. Child and Jungen use the common approach of expressing the asymmetric top rotational wavefunctions as a superposition of symmetric top wavefunctions where the molecular projection $\mathrm{K}$ is a good 
quantumnumber. A 'symmetrized case (b)' basis set is used where the electronic and rotational wavefunctions have parity-labels $\mathrm{p}_{e}$ and $\mathrm{p}_{\mathrm{r}}$.

At long range the electron is no longer coupled to the molecular frame and, the electron orbital angular momentum $l$ does not have a well-defined body-fixed projection $\lambda$, but a well-defined laboratory-fixed projection $m$. The molecular rotation is expressed in wave functions characterized by rotational quantum numbers $\mathrm{N}^{+}$, with projections $\mathrm{K}^{+}$in the molecular frame and laboratory frame projections $\mathrm{M}^{+}$, and the parity of the rotational wave function is $\mathrm{p}$. The electronic and rotational angular momenta give a total angular momentum $\mathrm{J}$ with a laboratory frame projection $\mathrm{M}$. Child and Jungen demonstrate how the transformation between the case (b) and case (d) modifies the selection rules which are normally appiicable to asymmetric top molecules. A discussion of these selection rules will be given in the next section.

\section{$\mathrm{H}_{2} \mathrm{O}$ Rydberg Spectroscopy}

The molecular orbital structure of the $\mathrm{H}_{2} \mathrm{O}$ molecule in its ground state is given by $X^{1} A_{1}\left\{\left(1 a_{1}\right)^{2}\left(2 a_{1}\right)^{2}\left(1 b_{2}\right)^{2}\left(3 a_{1}\right)^{2}\left(1 b_{1}\right)^{2}\right\}$. In the spectral range of interest in this chapter absorption features are observed corresponding to excitation of electrons in the $3 \mathrm{a}_{1}$ and the $1 b_{1}$ orbitals, which are a bonding orbital in the molecular plane and a nonbonding oxygen p-orbital pointing out of the molecular plane, respectively. Removal of an electron in the $1 b_{1}$ orbital forms the $\mathrm{H}_{2} \mathrm{O}^{+}$ion in the $\mathrm{X}^{2} \mathrm{~B}_{1}$ ground state, which has a bent geometry close to the initial $\mathrm{H}_{2} \mathrm{O}$ geometry. Removal of an electron from the $3 \mathrm{a}_{1}$ orbital forms the $\mathrm{H}_{2} \mathrm{O}^{+}$ion in the $\mathrm{A}^{2} \mathrm{~A}_{1}$ state, which is linear, and the large geometry change induces long progressions in the bending vibration. In the current chapter we will mainly be concerned with excitations of the $1 b_{1}$ orbital.

The first observation of members of the $\left(1 b_{1}\right)^{-1}$ Rydberg series discussed in this chapter was done by Price ${ }^{6}$, who measured members of four different Rydberg series labelled A, B, C and D, which were later interpreted as excitation from the nonbonding $1 b_{1}$ orbital to orbitals with symmetry $n p a_{1}(A), n p b_{1}(B), n d a_{1}$ and nda $(C)$, and $n \mathrm{db}_{1}$ and $\mathrm{nda}_{1}{ }^{\prime}$ (D) ${ }^{7}$. The quantum defects of these states are close to the 
quantum defects of a single oxygen atom, namely $\mu \approx 1.4$ for the ns series, $\mu \approx 0.7$ for the np series and $\mu \approx 0$ for the nd series, which matches reasonably well the oxygen atom quantum defects of $1.20,0.76$ and 0.03 respectively ${ }^{8)}$. In subsequent experiments the absorption and photoionization spectrum have been determined with increasing precision. In what follows, we will focus primarily on the observation of members of Rydberg series with principal quantum number $n \geq 6$.

Using a helium continuum source, Katayama et.al. ${ }^{9)}$ measured the total absorption and photoionization cross section in the waveiength range from 580 tc 1050 $\AA$ with a resolution of $0.5 \AA$, and were able to observe Rydberg series up to principal quantum number $n=8$. For these higher principal quantum numbers the spectrum is dominated by excitation to nd orbitals. Since the $1 b_{1}$ orbital is essentially an atomic $p$ orbital, the $\Delta l= \pm 1$ selection rules forbids excitation to p-orbitals unless the $\mathrm{np}$ Rydberg orbital is perturbed by the molecular core, as is the case for low principal quantum numbers. For higher principal quantum numbers the $n p<-1 b_{1}$ transition becomes nearly forbidden. Ab-initio calculations by Diercksen et.al. ${ }^{10)}$ show that the oscillator strength for excitation to nd levels is significantly larger than for excitation to ns levels, so to a good approximation the Rydberg series observed in the current experiments can be described as being due exclusively to nd <-- $1 b_{1}$ excitations. Using synchrotron radiation Ishiguro et.al. ${ }^{11)}$ and Gurtler et.al. ${ }^{12)}$ measured the absorption spectrum between $600 \AA$ and $1250 \AA$, with a resolution of $0.03 \AA$ in the latter experiment, and were able to determine the positions of the nd series out to principal quantum number $n=13$.

There are five different nd orbitals for each principal quantum number, namely nda $1\left(\sigma^{+}\right), n d b_{2}\left(\pi^{+}\right)$, nda $2\left(\pi^{-}\right)$, nda $_{1}^{\prime}\left(\delta^{+}\right)$and $n d b_{1}\left(\delta^{-}\right)$. Following the standard approach to evaluate the non-zero transition matrix elements for a dipole-allowed transition $^{13)}$, a transition moment along the $x$-axis (c-axis) transforms as $b_{1}$ and therefore c-type transitions $\left(\Delta \mathrm{K}_{\mathrm{f}}=\right.$ odd, $\Delta \mathrm{K}_{\mathrm{c}}=$ even) are allowed to nda $\mathrm{a}_{1}$ orbitals. A transition moment along the $y$-axis (a-axis) transforms as $b_{2}$ and therefore a-type transitions $\left(\Delta \mathrm{K}_{\mathrm{c}}=\right.$ even, $\Delta \mathrm{K}_{c}=$ oddd $^{\circ}$ are allowed to $\mathrm{a}_{2}$ orbitals. And finally, a transition moment along the z-axis (b-axis) transforms as $a_{1}$ and therefore b-type transitions $\left(\Delta \mathrm{K}_{\mathbf{z}}\right.$ 
$=$ odd, $\Delta \mathrm{K}_{\mathrm{c}}=$ odd) are allowed to $\mathrm{b}_{1}$ orbitals. There are no dipole-allowed transitions to orbitals of $b_{2}$ symmetry.

The quantum defect treatment of Child and Jungen showed that for high principal quantum numbers, in the limit of $l$-uncoupling, when the Rydberg electron is more appropriately described in a space-fixed coordinate system (Hund's case (d)) than in a molecule-fixed coordinate system (Hund's case (b)), the frame transformation from case (b) to case (d) results in an alternative set of propensity rules ${ }^{2}$ :

$$
\begin{gathered}
\lambda^{\prime \prime} \text { evenlodd } \rightarrow K_{a}^{+}-K_{a}^{\prime \prime} \text { evenlodd } \\
p_{c}^{\prime \prime}+\lambda^{\prime \prime}+l \text { even/odd } \rightarrow K_{c}^{+}-K_{c}^{\prime \prime} \text { evenlodd }
\end{gathered}
$$

where $\lambda^{\prime \prime}$ is the projection of the orbital angular momentum $l$ in the molecular frame in the ground electronic state and $\mathrm{p}_{e}$ " is the parity of the electronic wavefunction in the ground electronic state . For the $1 b_{1}$ lone pair orbital of the $\mathrm{H}_{2} \mathrm{O}$ molecule, one has $\lambda$ " $=l^{\prime \prime}=1$ and $\mathrm{p}_{e}{ }^{\prime \prime}=0$, leading to $\Delta \mathrm{K}_{\mathrm{a}}=$ odd and $\Delta \mathrm{K}_{\mathrm{c}}=$ even, which corresponds to ctype transitions. In the Rydberg spectra of the $\mathrm{H}_{2} \mathrm{O}$ molecule a change in appearance is therefore anticipated between transitions to Rydberg levels with low principal quantum numbers, where a-type, b-type and c-type transitions are observed, and to Rydberg levels with high principal quantum numbers, where only the c-type transitions are expected to occur, and where consequently the number of observed rotational transitions will be reduced, in apparent agreement with the results of Page et. al.". Measurements of $\mathrm{H}_{2} \mathrm{O}$ Rydberg spectra with rotational resolution have been limited sofar. Fairly extensive data is available for the $3 \mathrm{~d}$ complex ${ }^{14,15,16,17)}$, and in fact a theoretical analysis for this portion of the spectrum was recently published ${ }^{18}$. For Rydberg excitations with higher principal quantum numbers to date the only published spectra with rotational resolution remain the photoionization results of Page et.al. ${ }^{1}$, which, using an extreme ultra-violet (xuv) laser with a $1.5 \mathrm{~cm}^{-1}$ bandwidth, show peaks corresponding to auto-ionization of individual rotational features of several 
Rydberg series converging on the (100) vibrational state of $\mathrm{H}_{2} \mathrm{O}^{+}$. The spectra published by Page et.al. show a fair amount of rotational structure for principal quantum number $n=6$, whereas for higher principal quantum numbers a significant amount of the rotational structure is lost in the continuum background.

Tonkyn et.al. ${ }^{19}$ have recently published the rotationally resolved threshold photoionization spectnum of the $\mathrm{H}_{2} \mathrm{O}$ molecule by using pulsed-field ionization of extremely high- $n$ Rydberg states ( $n \geq 150$ ). This spectrum shows the convergence limits for individual rotational transitions. A simulation of the intensities observed for different final rotational states of the $\mathrm{H}_{2} \mathrm{O}^{+}$ion revealed that the Rydberg excitation is described by a combination of a-type and c-type transitions. Possible explanations for the occurrence of a-type transitions, which contradict the above propensity rule, include l-mixing in the initial $1 b_{1}$ orbital or in the nominal nd orbital. Tonkyn et.al. ${ }^{19)}$ favoured the former, but this is ruled out by Gilbert and Child ${ }^{20)}$ on grounds that the np<--1b, ZEKE spectrum arising from a $d$ component in the initial orbital occurs at a higher frequency than the observed nd<--1 $b_{1}$ spectrum. Ab initio based studies by Lee et.al. ${ }^{21)}$ show conclusively that the main source of a-type intensity is $l$-mixing in the excited photoorbital. The dipole perturbation model offered by Gilbert and Child ${ }^{20)}$, which reaches the same conclusion by a different route, attributes the high intensity of the a-type ZEKE lines to intensity borrowing from moderate $n=50$ members of the allowed $n d<-1 b_{1}$ series which are imbedded in the dense near continuum of an $n p$ series terminating on a lower rotational level of the positive ion. Resonant mixing of this type is unlikely to affect the present low $n=10$ region of the spectrum, but persistent weak 1 -mixing can have a more subtle effect by altering the orbital composition of the excited state and hence breaking the propensity rule in Equation (6).

Wang et.al. ${ }^{2)}$ have otierved how in portions of the spectrum intensity is missing for excitation from the $1 b_{1}$ orbital to $n s, n p$ and nd orbitals of $a_{1}$ symmetry. They speculated that this is due to dynamic Renner-Teller interactions between bent and linear states with different values of the principal quantum number but similar energies. A recent paper by Hirst and Child ${ }^{23)}$ supports this idea. Hirst and Child calculated ab-initio bending potential curves for Rydberg states of $\mathrm{H}_{2} \mathrm{O}$ up to energies 
of about $12 \mathrm{eV}$. Their results indicate that the bending potentials contain a large number of avoided crossings between diabatic curves corresponding to bent states, describing excitation of the $1 b_{1}$ ncn-bonding orbital and diabatic curves corresponding to linear states, related to excitation of the $3 a_{1}$ bonding orbital. Interactions with the $3 \mathrm{pb}_{2}<--3 \mathrm{a}_{1}\left({ }^{\prime} \mathrm{B}_{2}\right)$ linear state, for which several low-lying bending levels have been reported $^{24)}$, are predicted to be particularly important, because the avoided crossing are predicted to lie close to all nda $2<-1 b_{1}\left({ }^{1} B_{2}\right)$ potential minima and a strong perturbation of this type has already been observed in the $E^{\prime}$ band of the $3 d<--1 b_{1}$ cluster ${ }^{18)}$.

Once formed, the fate of the Rydberg molecule depends strongly on whether its total energy does or does not exceed the ionization threshold. Levels below the ionization threshold are predissociated by the $\mathbf{B}^{1} \mathbf{B}_{1}$ state and the asymptotic channels which are energetically accessible are ${ }^{25)} \mathrm{OH}\left(X^{2} \Pi\right)+\mathrm{H}(n=1), \mathrm{OH}\left(\mathrm{A}^{2} \Sigma^{+}\right)+\mathrm{H}(n=1)$, $\mathrm{H}_{2}\left({ }^{1} \Sigma_{8}^{+}\right)+\mathrm{O}\left({ }^{3} \mathrm{P}\right), \mathrm{H}_{2}\left({ }^{1} \Sigma_{\mathrm{z}}^{+}\right)+\mathrm{O}\left({ }^{1} \mathrm{D}\right)$ and $\mathrm{O}\left({ }^{3} \mathrm{P}\right)+2 \mathrm{H}(\mathrm{n}=1)$. For example, at an excitation energy of $10.2 \mathrm{eV} 78 \%$ of the Rydberg molecules decay into $\mathrm{H}+\mathrm{OH}, 12$ $\%$ into $\mathrm{H}+\mathrm{H}+\mathrm{O}$ and $10 \%$ into $\mathrm{H}_{2}+\mathrm{O}^{26}$. Above the ionization threshold autoionization becomes a major decay channel and Dutuit et. al. ${ }^{25)}$ observed an approximately $50 \%$ drop in the fluorescence of the $\mathrm{OH}\left(\mathrm{A}^{2} \mathrm{\Sigma}^{+}\right)$state around the ionization threshold.

\section{Experimental Approach}

The experimental apparatus used in this experiment consisted of a near-perpendicular crossed beam arrangement of an extreme ultra-violet (xuv) laser and a pulsed molecular beam, which will now be described in turn.

Fulsed xuv radiation was generated by frequency-upconversion of the output of a near transform-limited visible dye laser system, which is based on pulseamplification of the continuous output of a Coherent 699-29 Autoscan ringlaser in four stages, using three prism dye amplifiers, where the second dye amplifier is doublepassed ${ }^{28)}$. The dye-amplifiers are pumped by the second harmonic of a Quantel $\mathrm{Nd:YAG}$ laser model 581C. At a $600 \mathrm{~nm}$ wavelength this laser system is capable of 
generating up to $120 \mathrm{~mJ}$ in a $7 \mathrm{nsec}$ FWHM pulse with a near transform-limited bandwidth of $91 \mathrm{MHz}$. The laser operates at a $10 \mathrm{~Hz}$ repetition rate.

The dye laser output was frequency-doubled using KDP crystals, generating in excess of $30 \mathrm{~mJ} /$ pulse of ultra-violet radiation. This ultra-violet radiation was focussed onto a pulsed Ar jet using a $25 \mathrm{~cm}$ focal length fused silica lens and xuv radiation was obtained by frequency tripling ${ }^{29)}$. A home-built pulsed valve according to the design of Proch and Trick ${ }^{30)}$ was used to generate the pulsed Ar jet, and was operated with a 50 psig Ar backing pressure behind a $1 \mathrm{~mm}$ nozzle. The tripling chamber was differentially pumped by a 6" Varian cryopump. The xuv output of the entire laser system was calibrated by Cromwell et.al. at $98 \mathrm{~nm}$, where it was reported to exceed $10^{11}$ xuv photons/pulse ${ }^{28)}$. The bandwidth of the xuv laser was experimentally determined to be $210 \mathrm{MHz}$ by studying $(1+1)$ photoionization of $\mathrm{Kr}$ atoms ${ }^{31}$.

The xuv radiation passed through a 1 meter McPherson Model 225 vuv monochromator, which served two purposes. First of all, by operating the grating in first order, the monochromator allowed separation of the xuv and the co-propagating ultra-violet radiation, so the direct photoionization experiments could be carried out without the possibility of multi-photon interferences from the ultra-violet, which is about five orders of magnitude more intense than the xuv light. And secondly, the grating serves to refocus the xuv radiation, which is strongly divergent as it exits the tripling region. Since the tripling jet is located outside the monochromator, the xuv radiation is refocussed inside the monochromator when a standard grating for this monochromator is used, and at the crossing point with the molecular beam in the main chamber the xuv beam size is about $3 \mathrm{~mm}$, which approximately matches the size of the molecular beam. This is the condition under which the direct photoionization measurements were performed.

In the $(1+1)$ multi-photon ionization (MPI) experiments the grating was operated in zeroth order, where the ultra-violet light which had been used to generate the xuv radiation served to ionize the Rydberg molecule. In order to achieve this it was necessary however to change the experimental arrangement a little. The $\mathrm{H}_{2} \mathrm{O}$ Rydberg states studied in this experiment are rapidly predissociated, with lifetimes of 
about 10 picoseconds. If the ultra-violet light is refocussed into a $3 \mathrm{~mm}$ spot the ionization rate of the Rydberg molecules is too low to give a detectable signal, and therefore the 1 meter focal length grating was replaced by a 1.188 meter focal length grating which was mounted off the backflange of the vuv monochromator and manipulated in such a way that the xuv and ultra-violet were refocussed at the molecular beam. The MPI signals that were obtained in this fashion were up to ten percent of the signals in the direct photoionization experiment.

The pulsed $\mathrm{H}_{2} \mathrm{O}$ molecular beam was generated by using a second pulsed valve according to the design of Proch and Trick ${ }^{30}$. The $\mathrm{H}_{2} \mathrm{O}$ sample was held at $\mathrm{O}{ }^{\circ} \mathrm{C}$, and expanded through a $1 \mathrm{~mm}$ nozzle in 150 Torr of Ne carrier gas. The pulsed valve was located inside a separate source chamber, and the pulsed molecular beam was formed by skimming the pulsed $\mathrm{H}_{2} \mathrm{O}$ jet $4 \mathrm{~cm}$ from the nozzle with a $2 \mathrm{~mm}$ ID skimmer. The distance from the nozzle to the laser axis was about $8 \mathrm{~cm}$. The rotational temperature of the $\mathrm{H}_{2} \mathrm{O}$ beam is not accurately known, but is probably not too different from the

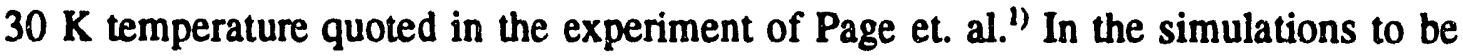
discussed in the next section a $25 \mathrm{~K}$ beam temperature was assumed. Ions formed at the crossing point of the laser and the molecular beam were mass selected by time-offlight and detected by using a Daly-type detector. The signal from the detector was fed into a boxcar averager and stored on the Autoscan computer.

Shot-to-shot fluctuations in the xuv laser power can easily be as much as $50 \%$, and therefore, in order to obtain a good signal-to-noise ratio in the direct photoionization experiment, a normalization procedure for the xuv laser power was required. Similar to Page et. al. ${ }^{1)} \mathrm{C}_{2} \mathrm{H}_{2}$ was mixed in with the Ne carrier gas to provide a nearly wavelength independent ionization signal with the same laser power dependence as the $\mathrm{H}_{2} \mathrm{O}$ photoionization signal. The $\mathrm{H}_{2} \mathrm{O}^{+}$and $\mathrm{C}_{2} \mathrm{H}_{2}{ }^{+}$signals were averaged separately on two boxcar averagers and the ratio, determined by an analog processor, was used as a true measure of the $\mathrm{H}_{2} \mathrm{O}$ photoionization efficiency. In order to achieve the optimum linearity for the normalization procedure, the $\mathrm{Ne} / \mathrm{C}_{2} \mathrm{H}_{2}$ mixture was prepared with a $\mathrm{Ne}: \mathrm{C}_{2} \mathrm{H}_{2}$ partial pressure ratio of about $100: 1$, which resulted in similar signal levels at the $\mathrm{H}_{2} \mathrm{O}^{+}$and $\mathrm{C}_{2} \mathrm{H}_{2}{ }^{+}$peaks. The single shot variations in the 
normalized signal were about $20 \%$, with no residual correlation to the xuv laser power. Spectra were recorded by scanning the Autoscan ringlaser and averaging the signal obtained from 30 lasershots. All scans were repeated 3-5 times, and thus the photoionization spectrum was obtained with a signal-to-noise ratio of about 50:1, allowing many weak rotational features, which could not be observed before in the experiment of Page et. al. ${ }^{1}$, to be resolved. It should be mentioned that the normalization with $\mathrm{C}_{2} \mathrm{H}_{2}$ also takes care of the wavelength dependence of the laser power and the Ar tripling efficiency.

In the MPI experiment, the $\mathrm{C}_{2} \mathrm{H}_{2}$ can be ionized either by absorption of a single xuv photon or by absorption of three or more photons from the ultra-violet laser which co-propagates with the xuv laser. When the efficiency of the xuv generation was optimized, it was found that the signal for the direct photoionization by one xuv photon was about 15 times stronger than the latter MPI process, and therefore the MPI spectra were recorded applying the normalization as well. Once an $\mathrm{H}_{2} \mathrm{O}^{+}$signal was found in the MPI experiment, the location of focus of the xuv laser was fine-adjusted by simultaneously moving the nozzle of the Ar jet and the $25 \mathrm{~cm}$ lens which focusses the ultra-violet light onto the jet along the laser propagation axis.

\section{Results and Analysis}

(1+1) Multi-photon ionization (MPI) spectra were recorded from $98550 \mathrm{~cm}^{-1}$ up to the ionization threshold at $101766 \mathrm{~cm}^{-1}{ }^{19}$, corresponding to the frequency range of the Rydberg series ( $n d<--1 b_{1} ; n \geq 6$ ), converging on the (000) vibrational state of $\mathrm{H}_{2} \mathrm{O}^{+}$. A typical portion of the spectrum, showing absorption features in the spectral region of the $6 \mathrm{~d}<-1 \mathrm{~b}_{1}$ transitions, is reproduced in the upper panel of Figure 1a. Single-photon ionization spectra were recorded from $101610 \mathrm{~cm}^{-1}$ to $104120 \mathrm{~cm}^{-1}$, corresponding to the frequency rarge of the autoionizing Rydberg series (nd $<-1 b_{1} ; n=6-11$ ), converging on the (100) vibrational state of $\mathrm{H}_{2} \mathrm{O}^{+}$at $104979 \mathrm{~cm}^{-1}$. A typical section of the spectrum, showing the photoionization efficiency in the vicinity of the $6 d<--1 b_{1}$ transitions, is shown in the upper panel of Figure 2a. Positions and intensities for the 
dominant observed transitions are summarized in Tables 1 and 2, along with results of the quantum defect calculations to be described below.

In relation to Figures $1 \mathrm{a}$ and $2 \mathrm{a}$, a few introductory remarks can be made. First of all the direct photoionization and the MPI spectrum have a significant number of lines in common, illustrating the fact that (i) the quantum defects for the direct photoionization and the MPI experiment should be quite similar, and (ii) the rotational structure for $\mathrm{H}_{2} \mathrm{O}^{+}(000)$ and $\mathrm{H}_{2} \mathrm{O}^{+}(100)$ is almost identical. The differences between Figures $1 \mathrm{a}$ and $\mathrm{lb}$ point towards perturbations affecting the $6 \mathrm{~d}$ levels in one or both of the Rydberg series and towards differences in the decay mechanisms for the $6 \mathrm{~d}$ members of both series.

In the MPI results in Figure 1a the linewidths are typically $O\left(1 \mathrm{~cm}^{-1}\right)$, whereas in the photoionization results the linewidths are typically $O\left(2-3 \mathrm{~cm}^{-1}\right)$. This is consistent with the fact that below the ionization threshold the linewidth is determined predominantly by the lifetime towards predissociation, whereas above the ionization threshold the linewidth is determined by the competing processes of auto-ionization and predissociation. This result is consistent with the experiments of Dutuit et. al. ${ }^{25}$ which were mentioned in the introduction.

As noted by Page et.al. ${ }^{1)}$, for each principal quantum number value, the spectra tend to be dominated by two peaks, which Page et. al. labelled ' $a$ ' and ' $b$ ' in their photoionization spectra. From their MQDT calculation ${ }^{2)}$ Child and Jungen concluded that the a-series corresponded to excitation from the $1_{01}$ ground rotational state to a Rydberg level corresponding predominantly to a $1_{11}$ ionic rotational state, whereas the b-series corresponded to excitation from the $1_{01}$ ground rotational state to a Rydberg level corresponding predominantly to a $2_{11}$ ionic rotational state. In the current experiment the na-nb series can be followed to $n=13$ in the MPI results and to $n=11$ for the direct photoionization results.

It is interesting to look at the linewidths of the peaks of the na/nb series. In the current experiments the laser bandwidth is negligible compared to any of the observed linewidths and therefore these linewidths give a direct determination of the lifetime of the Rydberg level which is excited. This statement is also valid for the MPI 
Figure 1 Observed (upper panel) and simulated (lower panel) MPI spectra for $\mathrm{n}=6-12$. The simulation employs a Lorentzian profile with $\mathrm{FWHM}=1.0$ $\mathrm{cm}^{-1}$. Assignments given for $\mathrm{n}=6$ represent the dominate case (b) component of the wavefunction. Those marked ? are too heavily mixed by Coriolis coupling to be assignable. The designation $d_{2} 1_{1}$. etc. implies complete Coriolis coupling between the F components ${ }^{23)}$. 


$$
N=6
$$

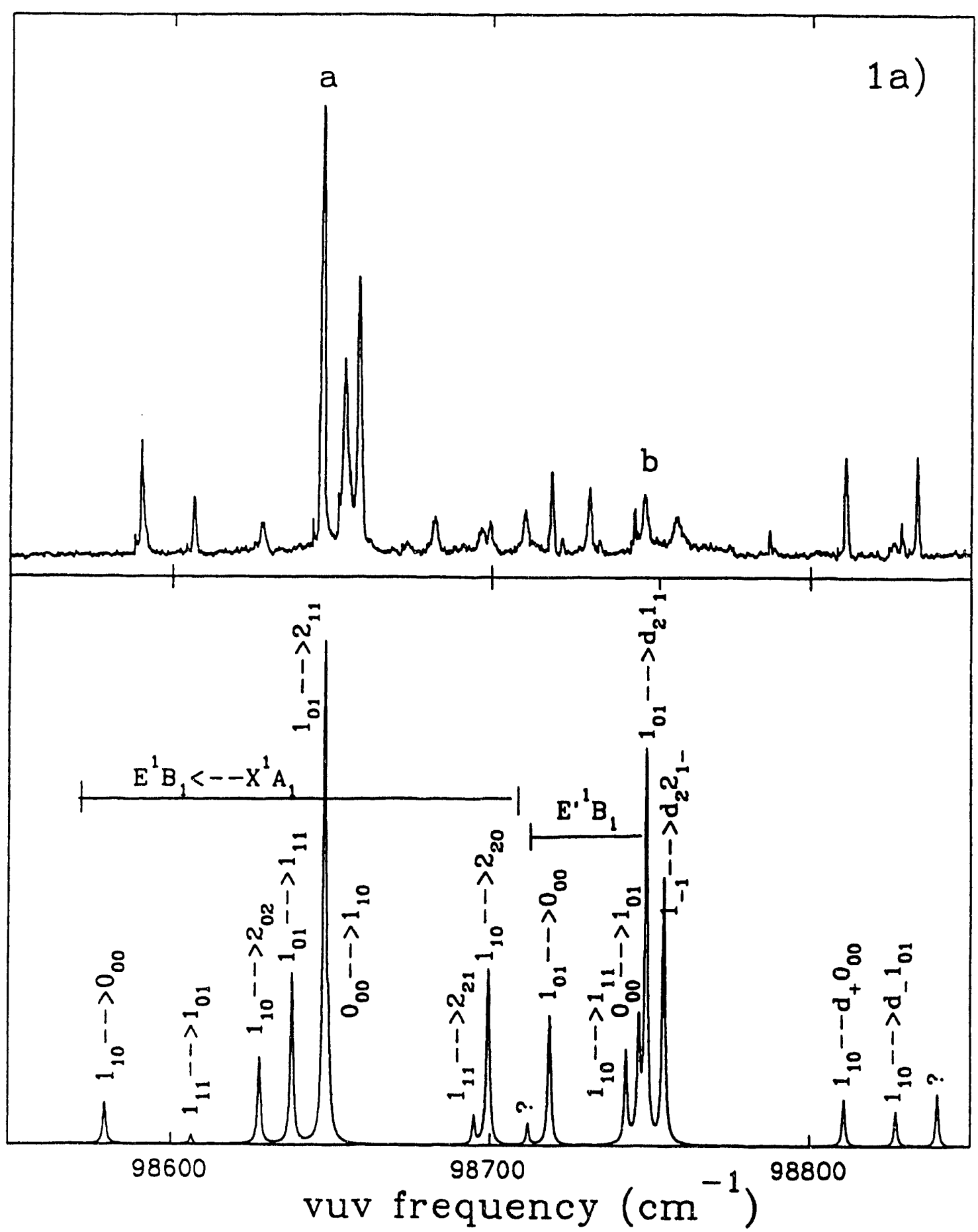


$N=7$

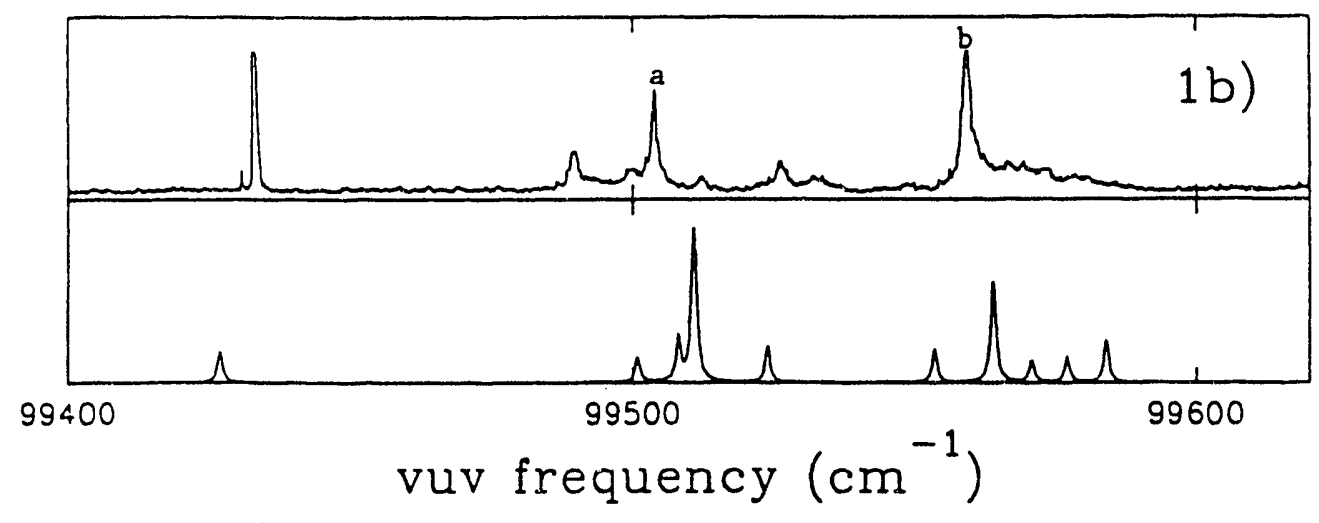

$$
N=8
$$

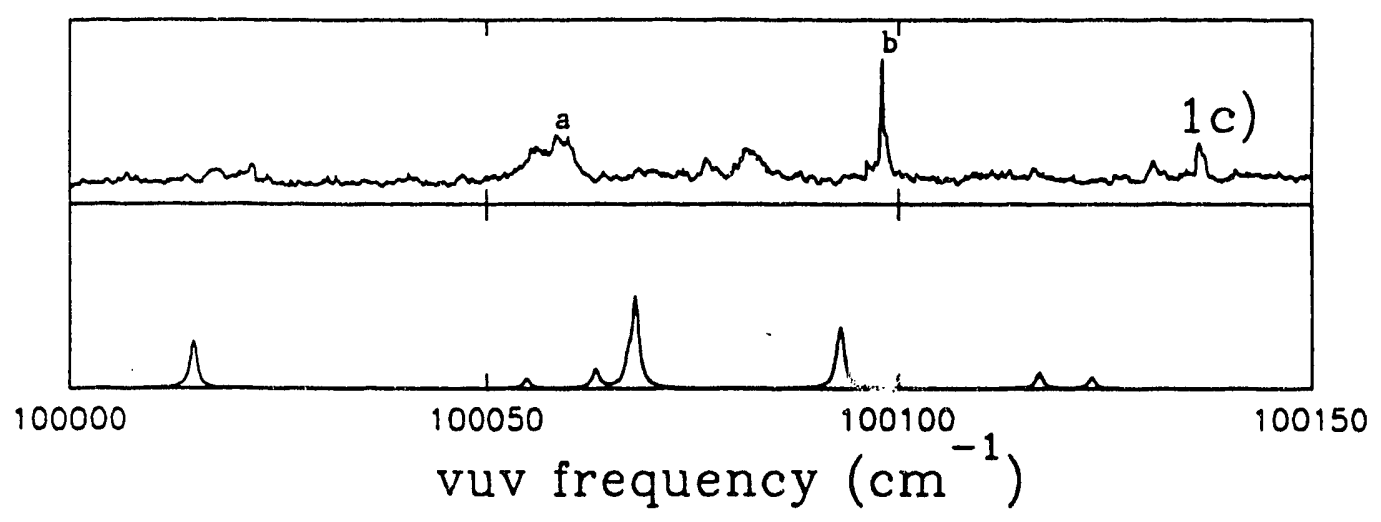

$$
N=9
$$

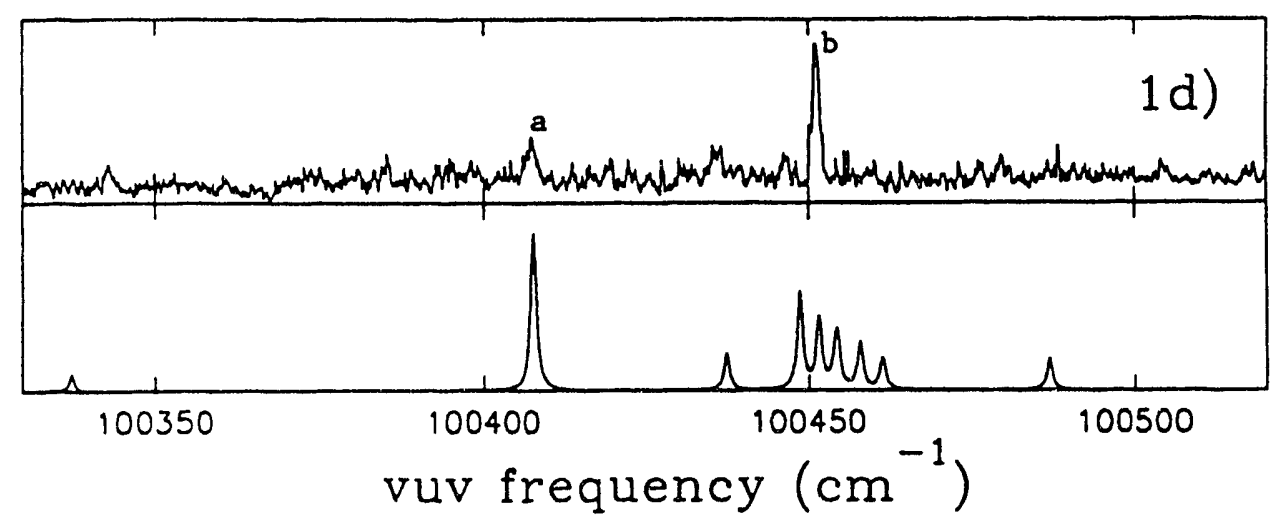




$$
N=10
$$

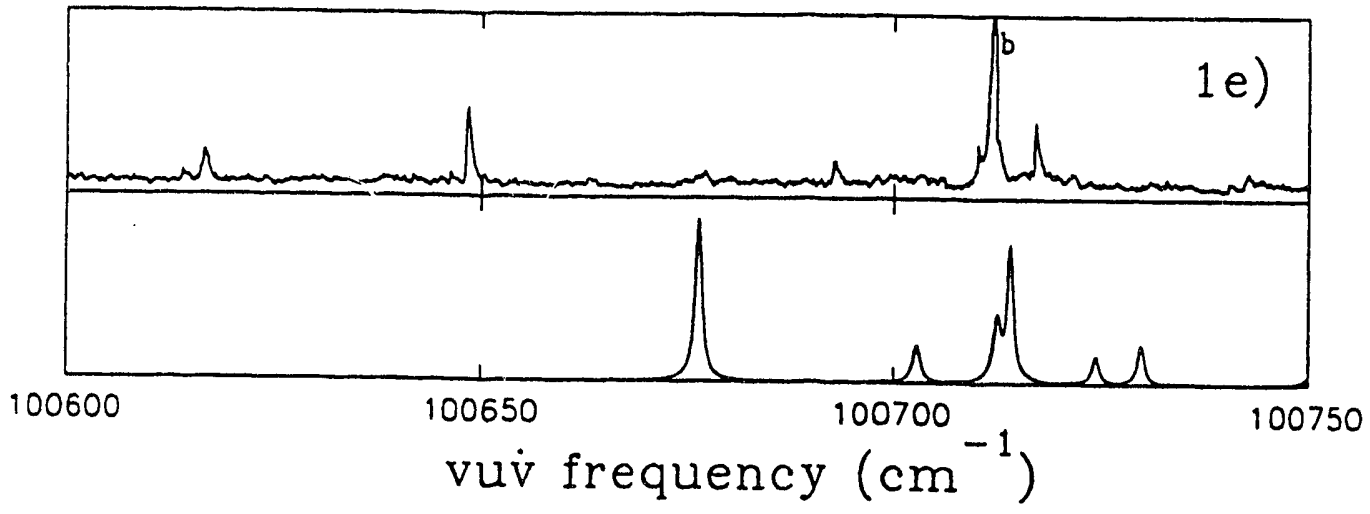

$$
N=11
$$

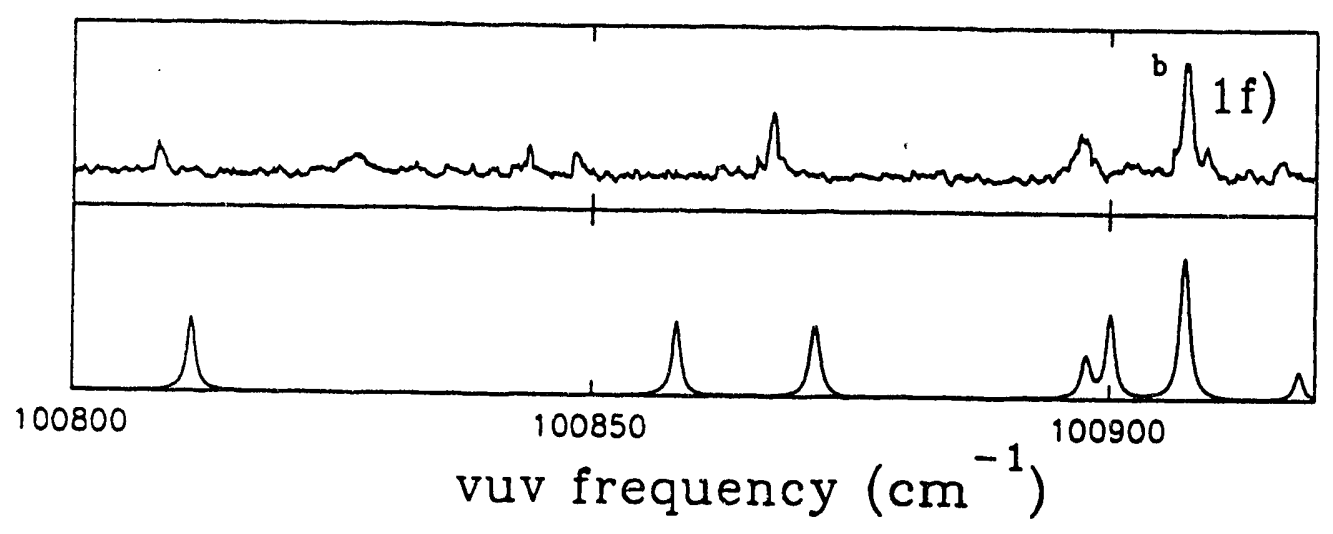

$$
N=12
$$

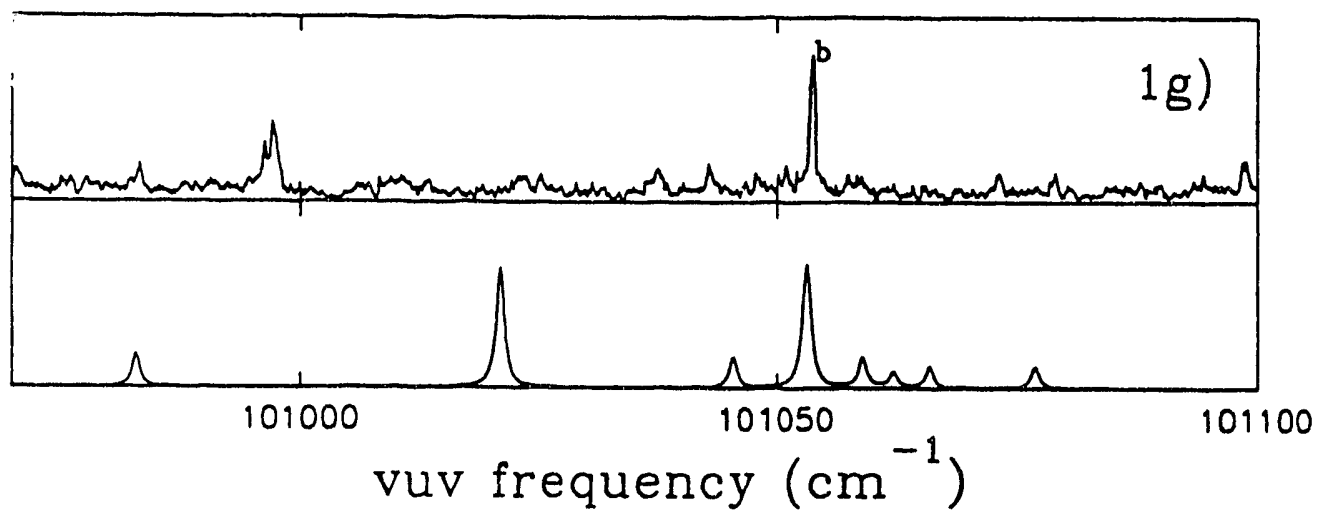


Figure 2 Observed (upper panel) and simulated (lower panel) photoionization spectra for $n=6-11,2 a$ to $2 f$ respectively, plus a case (d) simulation in figure $2 \mathrm{~g}$ obtained by switching off the $s / d \sigma$ mixing and by setting all quantum defects to zero. The simulation employs : Lorentzian profile with FWHM $2.0 \mathrm{~cm}^{-1}$. Assignments given for $n=6$ represent the dominate case (b) component of the wavefunction. The designation $d_{2} 1_{1}$. etc. implies complete Coriolis coupling between the two $\mathrm{F}$ components ${ }^{23}$. 
$N=6$

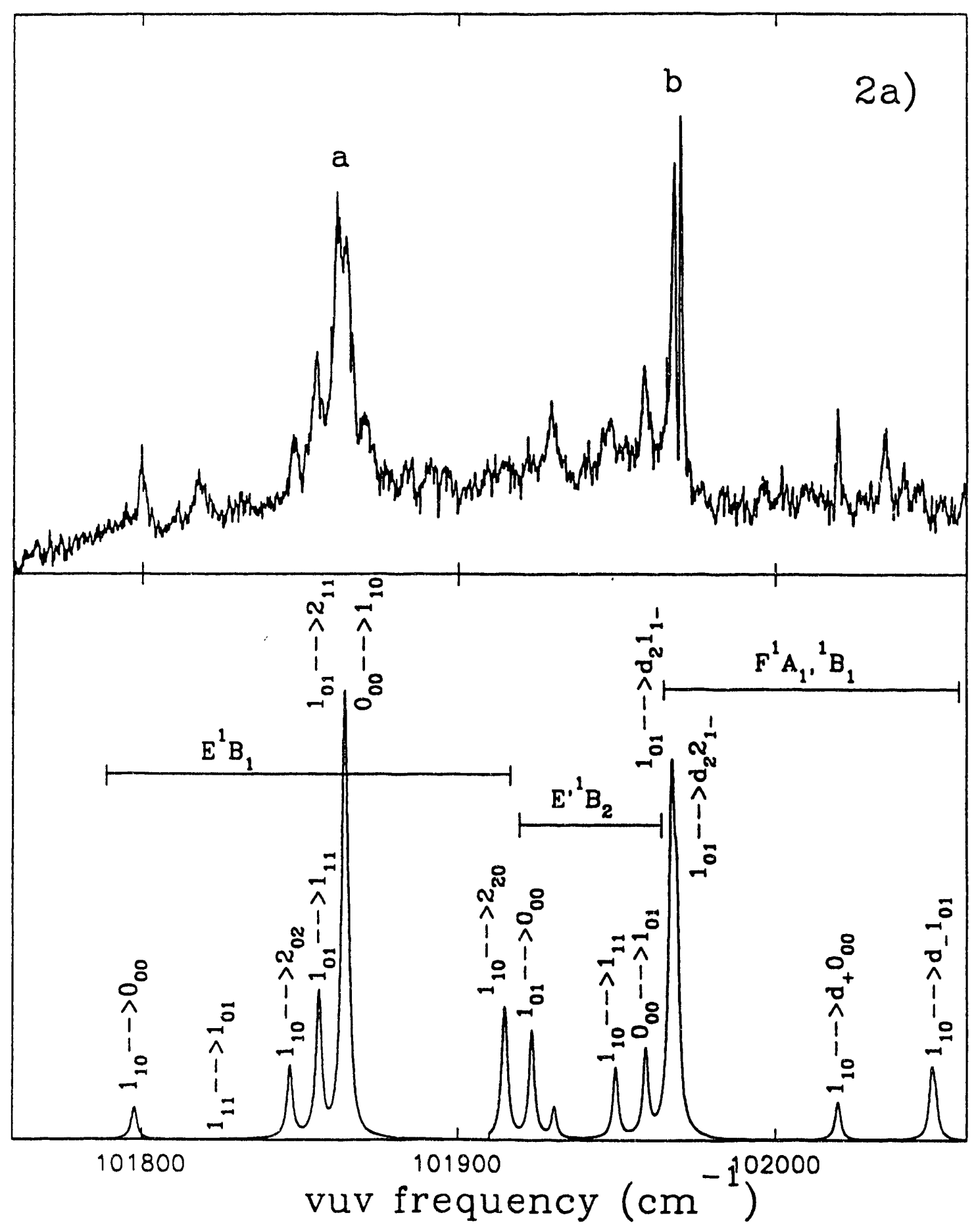




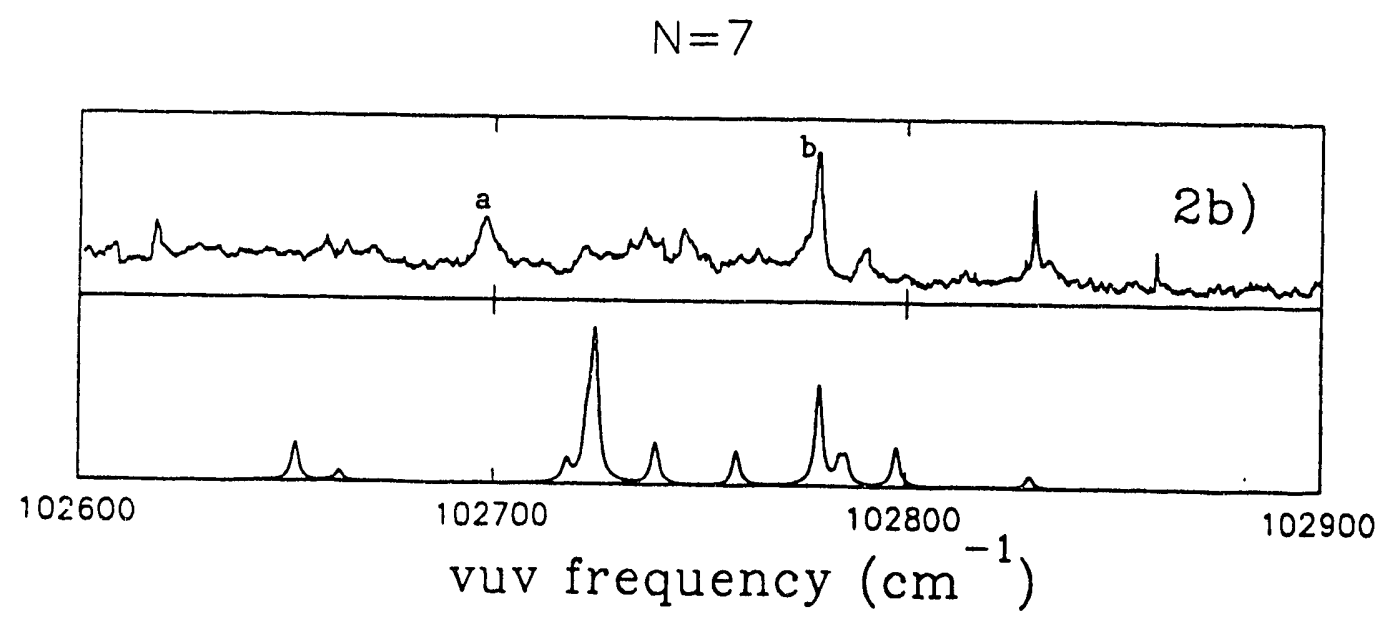

$$
N=8
$$

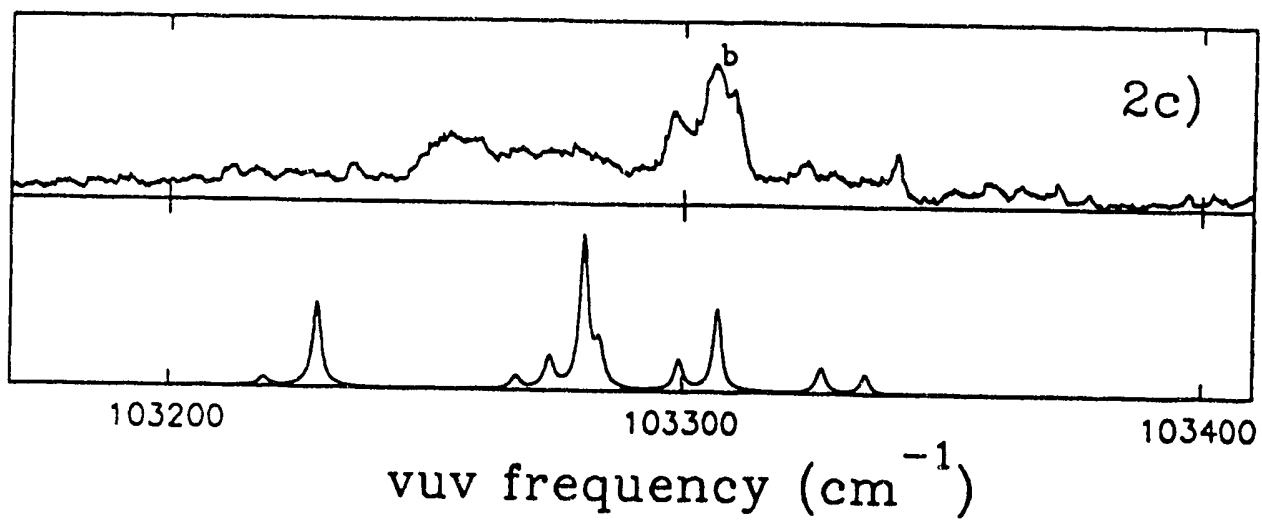

$$
N=9
$$

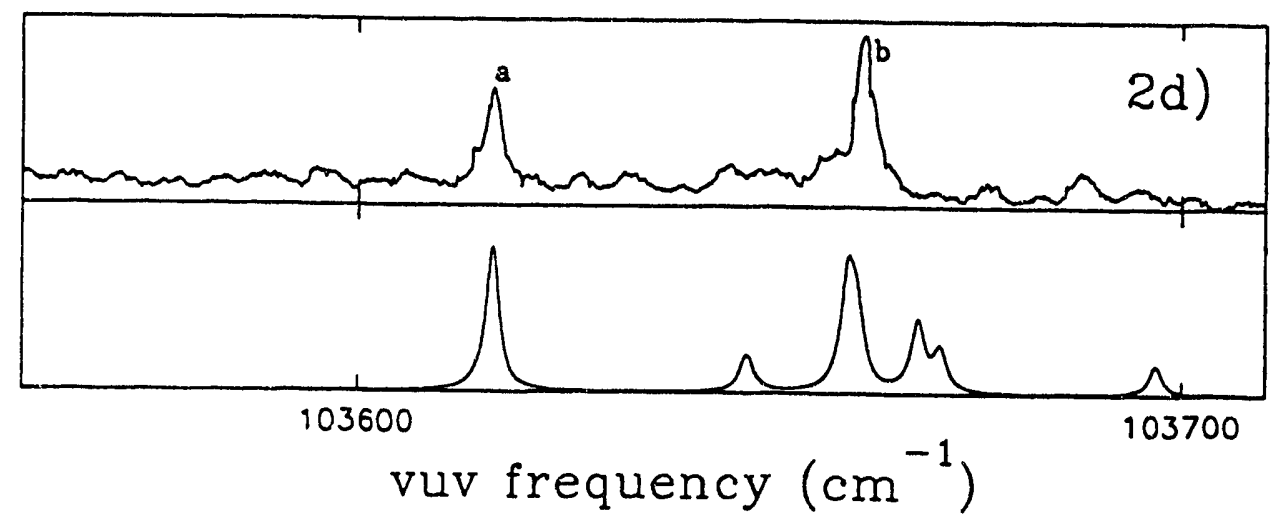




$$
N=10
$$

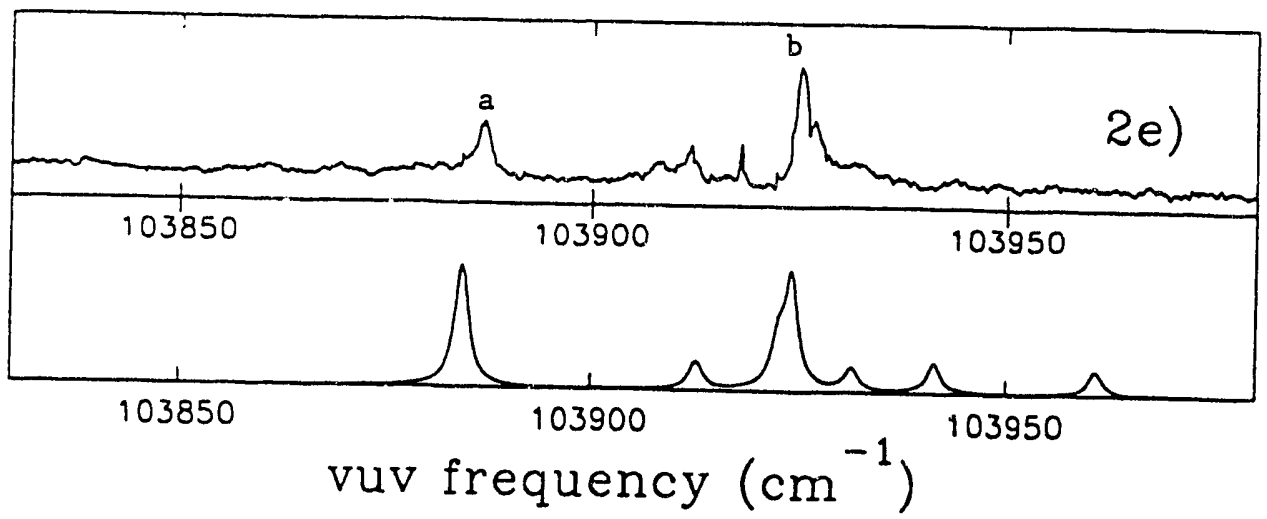

$$
N=11
$$

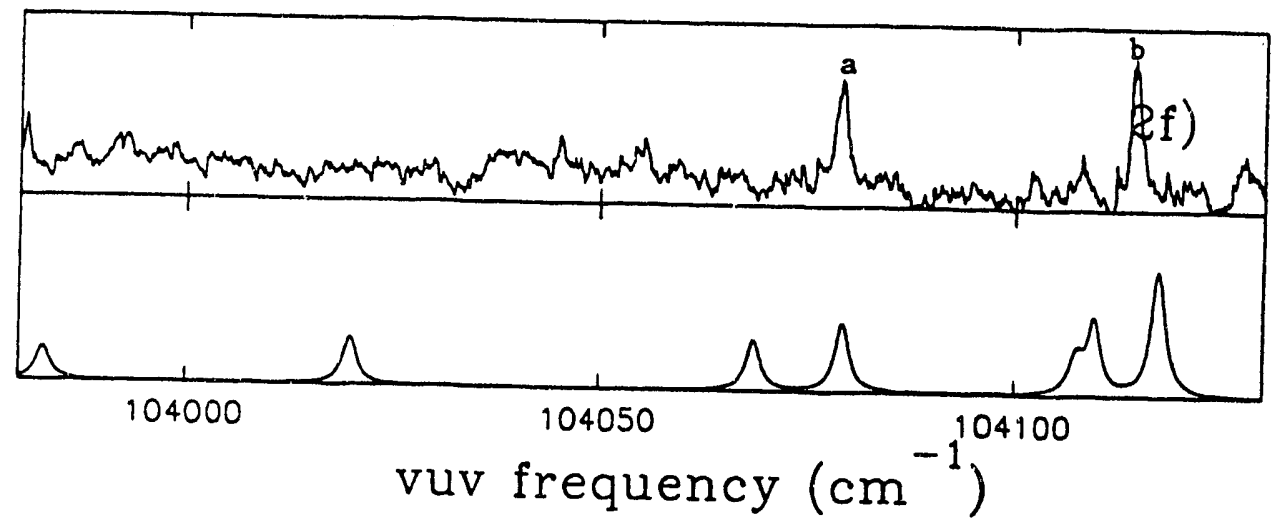

Case (d) simulation for $\mathrm{N}=12$

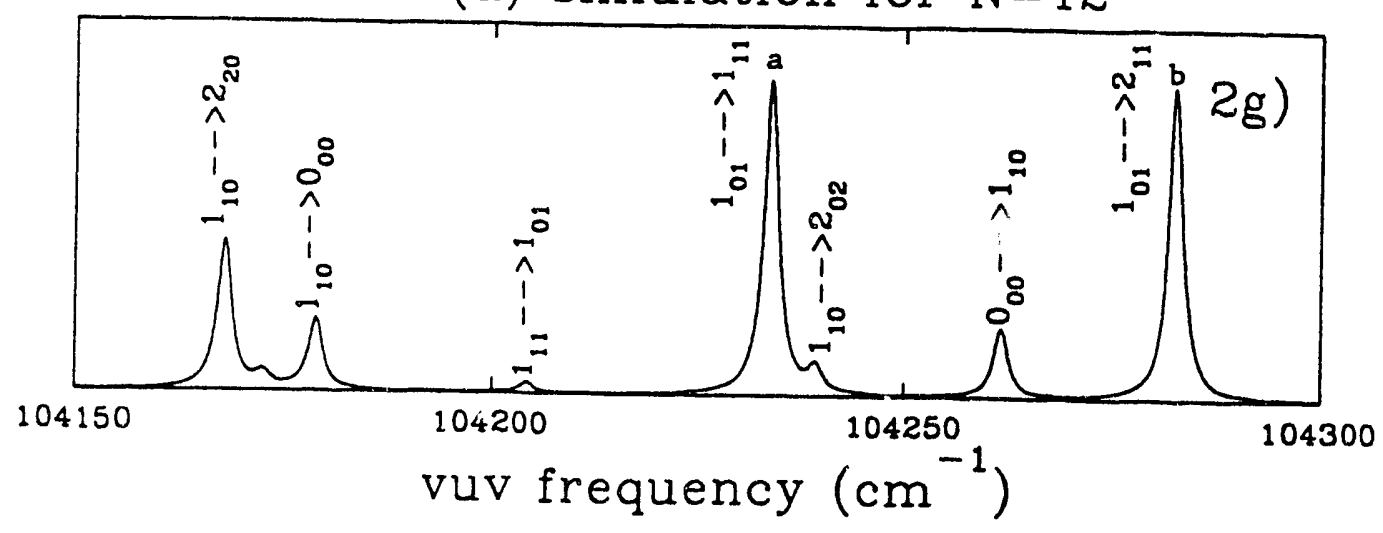


experiment, where an estimated $95 \%$ of the excited molecules decayed via predissociation. The observed linewidths are collected in Table 3. As expected, the linewidths for the Rydberg series converging on the $\mathrm{H}_{2} \mathrm{O}^{+}(000)$ vilurational state are lower than those for the Rydberg series converging on the $\mathrm{H}_{2} \mathrm{O}^{+}(100)$ vibrational state. Also, it is observed that the lines generally become narrower with increasing principal quantum number, reflecting the reduced core-penetration of the Rydberg orbital for highly excited Rydberg orbitals. Table 3 shows enhanced linewidths for some selected lines. These enhanced linewidths could in principle provide valuable information about interactions perturbing the Rydberg levels, such as interactions with linear states involving excitations of the $3 a_{1}$ orbital.

\section{Quantum Defect Analysis}

The observed MPI and photoionization spectra, toge her with a tentative multichannel quantum defect simulation ${ }^{2)}$, based on assignment to the nd(000) <- $1 b_{\text {; }}$ and ild(100) - $1 b_{1}$ series respectively, are shou. in Figures 1 and 2. Mincr differences $t$ atween the two series are to be expected from differences in the quantum defects, since in the two series the electron-core collisions sample a slightly different ange of ion-core geometries. The observed discrepancics are however too numerous and too unsystematic to be accounted for in this way, because in any case the simulated spectra for $n \geq 9$ are found to depend only on the composition of the channel wavefunctions (see below) and the $\mathrm{H}_{2} \mathrm{O}^{+}$rotational constants, and hardly at all on any reasonable choice of the quantum defects.

One obvious feature is that the $n=7$ and $n=8$ photoionization bands are heavily overlapped by other band systems, which might include bending levels of one or more of the $3 d<-3 a_{1}$ linear states as suggested by Page et.al. ${ }^{1)}$ or of the $3 p<-3 a_{1}$ linear states. In particular it should be noted that lower bending levels of the $3 \mathrm{pb}_{2}<--$ $3 a_{1}\left({ }^{1} B_{2}\right)$ linear state have already been reported ${ }^{24)}$, that one of them not only overlaps but also perturbs the $3 \mathrm{~d}$ cluster $^{20)}$ and that other possible interactions between the $3 \mathrm{pa}_{1}$ $<-3 a_{1}\left({ }^{\prime} A_{1}\right)$ and $3 p b_{1}<-3 a_{1}\left({ }^{\prime} B_{1}\right)$ linear states and higher members of the nd <-- $1 b_{1}$ 
series are suggested by a recent ab initio study ${ }^{23)}$.

A second possible explanation for the observed differences between Figures 1 and 2 lies in the method of detection, because lines may be lost from the MPI spectrum by rotationally selective predissociation, which is well attested elsewhere in the spectrum ${ }^{20.32}$. Similar loss is however less likely from the photoionization series because the autoionization quantum yield is known to exceed that for predissociation $^{25}$. Such selective predissociation could for example account for the intensity difference between the strong feature in the $n=6$ photoionization spectrum (labelled $b$ in Figure 2(a) after Page et.al. ${ }^{1}$ ) and the much weaker ' $b$ ' feature in the $\mathrm{n}=6 \mathrm{MPI}$ band. It is also difficult to account for the absence of strong ' $a$ ' feature lines in the MPI series for $n=10-12$ except by the opening up of a predissociation channel.

Turning to the details of the simulation, there is evidence for significant $l$ mixing in the fact that the spacings of the ' $a$ ' and ' $b$ ' peaks in the $n=9-11$ members of the photoionization series are markedly less than the $2_{11}<-1_{11}$ level separations in the simulated nd <-- $1 b_{1}$ case (d) spectrum in Figure 2(g). This can be understood in the following way. In the Watson Hamiltonian ${ }^{33)}$ the case (b) terms $\mid J(K \lambda\rangle^{18)}$ are Coriolis coupled by the terms $J_{a} l_{b}, J_{b} l_{b}$ and $J_{c} l_{c}$. However, if what is nominally a $|d \sigma\rangle$ orbital is actually an s/d admixture

$$
|d \sigma\rangle-\cos \theta|20\rangle+\sin \theta|00\rangle
$$

then any Coriolis coupling within the $d$ manifold is diminished by a factor $\cos \theta$. In the present case, the necessary reduction in Coriolis coupling to account for this diminished strong peak separation was most simply accommodated by allowing an admixture of $s$ character into the do orbital, with a mixing angle of $0.1 \pi$. Supporting evidence comes from the fact that such mixing would also enhance the abnormally low calculated intensity in the simulated $E$ component of the $3 d<--1 b_{1}$ cluster ${ }^{18)}$. No doubt there is also $\mathrm{p} / \mathrm{d}$ mixing in the excited state but it was less easy to find clear evidence of its likely magnitude. Hence only the above s/do mixing was taken into account, with an energy independent strength which was taken to be common to the two series. 
It should be noted in this context that Lee et.al. ${ }^{21)}$ find definitive evidence of $\mathrm{s} / \mathrm{p} / \mathrm{d}$ mixing in an ab initio based simulation of the ZEKE spectrum of $\mathrm{H}_{2} \mathrm{O}^{19}$. In particular p-wave contributions to the photoorbital account very well for the occurrence of type-a lines in the observed spectrum that are forbidden by the nd <-$1 b_{1}$ propensity rule ${ }^{2}$. Secondly, certain ZEKE lines have mixed s/d contributions to their intensity while others appear as almost pure $s<-1 b_{1}$ lines. The latter might be seen as limiting lines in the $n s<--b_{1}$ series, whose $n=3$ and $n=4$ members are known ${ }^{34,35)}$, except that the implied quantum defect, $\mu(s)=1.6$, would predict readily assignable ns $\left({ }^{1} B_{1}\right)<-X\left({ }^{1} A_{1}\right)$ bands well to the red of the complex $(n-1) d<-1 b_{1}$ bands in the present spectra. No such bands are observed; hence one must assume some mechanism for enhancing the ns <-- $\mathrm{lb}_{1}$ intensity (relative to the nd $<--\mathrm{lb}_{1}$ bands) as $\mathrm{n}$ tends to infinity.

Limiting case (b) and case (d) assignments are shown in Figures 1(a), 2(a) and $2(\mathrm{~g})$, but the assignment scheme used to optimize the quantum defects with an augmented $l$ mixed version of the model of Child and Jungen ${ }^{2)}$ was based on the only three conserved quantities - the total angular momentum $\mathrm{J}$ and two parity labels, termed $p$ and $p^{0}$ by Gilbert et al. ${ }^{18)}$. The quantum defects employed for the simulations, which are listed in Table 4, were separately optimized to the $n=6$ MPI and photoionization bands, whose close similarity suggests minimal effects from extraneous perturbations. Note however that the $n=6$ MPI assignment is ambiguous because two of the three strong lines around $98650 \mathrm{~cm}^{-1}$ and several weaker unassigned lines must be attributed to an overlapping system. Unfortunately the ambiguities cannot be resolved by use of combination differences because none are predicted at the assumed beam temperature of $25 \mathrm{~K}$. The assignment adopted was therefore based on the best fit, with standard deviation $\sigma=1.49 \mathrm{~cm}^{-1}$, subject to reasonable consistency with the photoionization quantum defects and those obtained in earlier studies ${ }^{2,18}$. Given the assignments, values for the two ionization limits were obtained by including observed strong $\mathrm{na}$ and $\mathrm{nb}$ lines for $\mathrm{n} \geq 9$ in the fit. The resulting values of $101773 \mathrm{~cm}^{-1}$ and $104985 \mathrm{~cm}^{-1}$ for the (000) and (100) limits agree well with the values of $101772 \mathrm{~cm}^{-1}$ and $104985 \mathrm{~cm}^{-1}$ obtained by Child and Jungen ${ }^{2}$, 
but differ significantly from the current best literature values of $101766 \mathrm{~cm}^{-1}{ }^{19)}$ and $104979 \mathrm{~cm}^{-1}$ obtained by adding $v_{1}=3213 \mathrm{~cm}^{-1}$ from Dinelli et. al. ${ }^{36)}$, presumably because the effects of $l$ mixing have only partly been taken into account. Tables 1 and 2 list comparisons between the observed and calculated line positions, except that many of the photoionization lines for $n=7$ and 8 and a long series of weak lines for $\mathrm{n}=9$, which are assumed to belong to overlapping series, have been omitted from the table.

The overall fit for the photoionization series is satisfactory given the presence of overlapping band for $n=7$ and $n=8$, which may also lead to bad perturbations. Differences between the quantum defects and those employed by Child and Jungen ${ }^{2)}$ arise from mino: differences in the assignments suggested by the higher resolution of the present spectrum and from the present incorporation of $s / d \sigma$ mixing. The situation with respect to the MPI series is less satisfactory. The assumption is that the strong na lines in the simulations for $n=10-12$ are removed by predissociation because their counterparts appear in the photoionization series and in the case (d) simulation. There is however only at best an accidental coincidence between the calculated and observed weak lines at these high $\mathbf{n}$ values. It is also extremely difficult to find a convincing simulation of the $n=8 \mathrm{MPI}$ band without assuming interaction with some presently unknown state.

Comparison with previous estimates for the quantum defects $\left.{ }^{2.18}\right)$ is complicated by the facts that the effects of s/do mixing were not previously taken into account, and that the $d \pi^{+}$defect is relatively ill-determined by the present data. There are however significant changes in the $(000)$ defects in passing from $n=3^{18)}$ to the present high $n$ values, particularly in the case of the do orbital, which would imply further changes as $\mathrm{n}$. increases. It is however unfortunately impossible to make sufficient confident assignments for high $\mathbf{n}$ members of the MPI series to test this contention. Differences between the high $\mathrm{n}(000)$ and $(100)$ defects are seen to lie outside the joint uncertainties only for the $d \sigma$ and $d \delta$ orbitals. These could be reconciled by shifts of 8 $\mathrm{cm}^{-1}$ and $19 \mathrm{~cm}^{-1}$ in the relevent $(000)$ electronic origins, of which the former could readily be accommodated by reassignment of the $6 \mathrm{a}$ line. Hence there is no definitive 
evidence from the $n>6$ bands of any significant difference between the $(000)$ and (100) quantum defects. Perceptible changes are however apparent between $n=3$ and $\mathrm{n}=6$.

Some of the uncertainties raised by this preliminary analysis could be removed by further experiments.

i) More definitive assignments could be made either by raising the beam temperature or by repeating the present experiments with $\mathrm{D}_{2} \mathrm{O}$ in order to allow the observation of ground state combination differences. Alternatively double resonance studies could be made via perhaps the well analysed ${ }^{37)} \mathrm{C}^{1} \mathrm{~B}_{1}$ state or by application of an ir-xuv ion depletion technique. Such improved assignments would be particularly valuable for the high $n$ MPI series because they might provide firm evidence about $d / p$ mixing in the excited state.

ii) It would be helpful to undertake a predissociation excitation search to yield $\mathbf{H}$ $+\mathrm{OH}(\mathrm{A})$ or $\mathrm{O}\left({ }^{1} \mathrm{D}\right)+\mathrm{H}_{2}$ in the region of the $\mathrm{nd}(000)<-1 b_{1}$ bands for $n=10-12$ in order to test the hypothesis that the strong na lines are lost by predissociation.

iii) Jet-cooled MPI experiments in the regions of the nd(000)<-- $1 b_{1}$ and nd(100) $<-1 b_{1}$ bands for $n=4$ and 5 would be very helpful in assigning congested room temperature absorption spectra taken by Mayhew ${ }^{38}$. It would then be possible to follow changes in the quantum defects with $\mathrm{n}$, in regions where the electronic band origins are most sensitive to such changes.

\section{Concluding remarks}

In this chapter new experimental results have been presented for one- and two-photon ionization of the water molecule involving resonant excitation to Rydberg levels converging on the (000) and (100) vibrational states of the $\mathrm{H}_{2} \mathrm{O}^{+}$ion, respectively. The experimental results have been analyzed by applying multichannel quantum defect theory (MQDT). The MQDT analysis suggests that Rydberg molecules in states with intermediately high principal quantum numbers, like the ones studied here, are near Hund's case (d), where the excited nd orbitals are near degenerate on the scale of the 
rotational constants. The nd components are still well separated from the ns and np components. Therefore, Coriolis coupling between the different ns, np and nd components is negligible. Within the nd manifold however, for $n=9-12$, the reduction in the Coriolis coupling towards higher $\mathrm{n}$ points towards some ndo/ns mixing. A pure case (d) spectrum is anticipated at even higher principal quantum numbers, when the separations between all electronic origins become negligible.

A comparison of the experimental results and the MQDT simulations shows that some serious discrepancies have remained. A few possible explanations have been offered. First of all, a comparison of the experimental results for $n=6$ in the one- and two-photon ionization, suggests that the two-photon ionization results, in which the molecule is initially excited below the first ionization threshold, may be subject to the influences of selective predissociation. And secondly, it is believed that a significant role in explaining the remaining discrepancies is played by the interaction between bent and linear Rydberg states. The ab-initio bending potential curves calculated by Hirst and Child ${ }^{23)}$ show a large number of avoided crossings between diabatic curves related to bent states describing excitations of the $1 b_{1}$ non-bonding orbital and diabatic curves related to linear states describing excitations of the $3 a_{1}$ bonding orbital. In particular, Hirst and Child pointed out that the diabatic bending potential energy curve for $3 \mathrm{pb}_{2}<-3 \mathrm{a}_{1}\left({ }^{3} \mathrm{~B}_{2}\right)$ excitation cuts through all the nda $<--1 \mathrm{~b}_{1}\left({ }^{1} \mathrm{~B}_{2}\right)$ diabatic bending potential energy curves close to their equilibrium bending angle. Since, as pointed out above, all the nd components of different symmetry are coupled through the Coriolis forces on the Rydberg electron, the $3 \mathrm{pb}_{2}<-3 \mathrm{a}_{1}\left({ }^{1} \mathrm{~B}_{2}\right)$ state would be a prime candidate to perturb the entire spectrum. Experimental credibility for this argument is given by the fact that both the positions and the lifetimes of some of the observed Rydberg progressions show irregular behaviour for certain values of the principal quantum number. Incorporation of the bent-linear interactions in a quantum defect treatment remains to be done. 


\section{REFERENCES}

1) R.H. Page and R.J. Larkin, Y.R. Shen and Y.T. Lee, J. Chem. Phys. $\underline{88}, 2249$ (1988).

2) M.S. Child and Ch. Jungen, J. Chem. Phys. 93, 7756 (1990).

3) M.J. Seaton, Rep. Prog. Phys. 46, 167 (1983).

4) U. Fano, Phys. Rev. 2,353 (1970).

5) Ch.H. Greene and Ch. Jungen, Adv. At. Mol. Phys. 21, 51 (1985).

6) W.C. Price, J. Chem. Phys. $\underline{4}, 147$ (1936).

7) W.A. Goddard III and W.J. Hunt, Chem. Phys. Lett. 24, 464 (1974).

8) M.B. Robin, "Higher Excited States of Polyatomic Molecules", (Academic, New York, 1974).

9) D.H. Katayama, R.E. Huffman and C.L. O'Bryan, J. Chem. Phys. 59, 4309 (1973).

10) G.H.F. Diercksen, W.P. Kraemer, T.N. Rescigno, C.F. Bender, B.V. McKoy, S.R. Langhoff and P.W. Langhoff, J. Chem. Phys. 76, 1043 (1982).

11) E. Ishiguro, M. Sasanuma, H. Masuko, Y. Morioka and M. Nakamura, J. Phys. B: Atom. Molec. Phys. 11, 993 (1978).

12) P. Gurtler, V. Saile and E.E. Koch, Chem. Phys. Lett. 51, 386 (1977).

13) P.R. Bunker, "Molecular Symmetry and Spectroscopy", (Academic, New York, 1979).

14) J.P. Connerade, M.A. Baig, S.P. McGlynn and W.R.S. Garton, J. Phys. B, 13 , L705 (1980).

15) C.A. Mayhew, PhD. thesis, Imperial College of Science, London (1984).

16) P.L. Smith, K. Yoshino, H.E. Griesinger and J.H. Black, Astrophy. J. 250, 166 (1981).

17) M.N.R. Ashfold and J.M. Bayley, J. Chem. Soc. Faraday. Trans. $\underline{86}, 213$ (1990).

18) R.D. Gilbert, M.S. Child and J.W.C. Johns, Mol. Phys. 74, 473 (1991).

19) R.G. Tonkyn, R. Wiedmann, E.R. Grant and M.G. White, J. Chem. Phys. 25 , 7033 (1991). 
20) R.D. Gilbert and M.S. Child, Chem. Phys. Lett. 287, 153 (1991).

21) M.-T. Lee, K. Wang, V. McKoy, R.G. Tonkyn, R.T. Wiedmann, E.R. Grant and M.G. White, J. Chem. Phys. 96, 7848 (1992).

22) H.-T. Wang, W.S. Felps and S.P. McGlynn, J. Chem. Phys. 67, 2614 (1977).

23) D.M. Hirst and M.S. Child, Mol. Phys. (in press).

24) E.H. Abramson, J. Zhang and D.G. Imre, J. Chem. Phys. 93,947 (1990)

25) O. Dutuit, A. Tabche-Fouhaile, I. Nenner, H. Frohlich and P.M. Guyon, J. Chem. Phys. 83, 584 (1985).

26) T.G. Schlanger and G. Black, J. Chem. Phys. 77, 2432 (19:82).

27) Ch. Jungen, K.E.J. Hallin and A.J. Merer, Mol. Phys, 40, 65 (1980).

28) a) E. Cromwell, T. Trickl, Y.T. Lee and A.H. Kung, Rev. Sci. Instrum. 60 , 2888 (1989).

b) E. Cromwell, PhD thesis, University of California at Berkeley (1991).

29) R.H. Page, R.J. Larkin, A.H. Kung, Y.R. Shen and Y.T. Lee, Rev. Sci. Instum. 58, 1616 (1987).

30) D. Proch and T. Trickl, Rev. Sci. Instrum. 60, 713 (1989).

31) T. Trickl, M.J.J. Vrakking, E. Cromwell, Y.T. Lee and A.H. Kung, Phys. Rev. A 39, 2948 (1989).

32) M.N.R. Ashfold, J.M. Bayley and R.N. Dixon, Chem. Phys. 84, 35 (1984).

33) J.K.G. Watson, J. Chem. Phys. $\underline{46}, 1946$ (1967).

34) G. Herzberg, "Electronic spectra of polyatomic molecules", (Van Nostrand, 1967).

35) M.N.R. Ashfold, J.M. Bayley and R.N. Dixon, Can. J. Phys. 62, 1806 (1984).

36) B.M. Dinelli, M.W. Crofton and T. Oka, J. Mol. Spectr. 127, 1 (1988).

37) J.W.C. Johns, Can. J. Phys. 49 , 944 (1971).

38) C. Mayhew (private communication). 


\begin{tabular}{|c|c|c|c|}
\hline \multicolumn{4}{|c|}{$\begin{array}{l}\text { Table 1: Observed and calculated MPI line positions. } \\
\text { Experimental lines marked }{ }^{*} \text { were used in the quantum defect optimization. }\end{array}$} \\
\hline $\begin{array}{l}\text { Experimental } \\
\text { line position }\left(\mathrm{cm}^{-1}\right)\end{array}$ & $\begin{array}{l}\text { Calculated } \\
\text { line position }\left(\mathrm{cm}^{-1}\right)\end{array}$ & $\begin{array}{l}\text { Calculated intensity } \\
\text { (a.u.) }\end{array}$ & $\begin{array}{l}\text { Assignment } \\
\left\{\mathrm{J}, \mathrm{p}, \mathrm{p}^{\circ}\right\}<-\mathrm{J}\left(\mathrm{K}_{\mathrm{c}} \mathrm{K}_{\mathrm{c}}\right)\end{array}$ \\
\hline \multicolumn{4}{|c|}{$\mathrm{n}=6$} \\
\hline 98589.94 & 98579.43 & 0.18 & $\{0,0,0\}<-1(10)$ \\
\hline 98606.80 & 98606.36 & 0.04 & $\{1,1,1\}<-1(11)$ \\
\hline \multirow[t]{2}{*}{$98628.16^{*}$} & 98627.80 & 0.38 & $\{2,0,0\}<-1(10)$ \\
\hline & 98638.21 & 0.75 & $\{1,0,1\}<-1(01)$ \\
\hline $98647.26^{*}$ & 98648.42 & 2.17 & $\{2,0,1\}<-1(01)$ \\
\hline 98647.26 & 98649.71 & 0.39 & $\{1,1,0\}<-0(00)$ \\
\hline \multicolumn{4}{|l|}{98654.43} \\
\hline \multicolumn{4}{|l|}{$9865 i .57$} \\
\hline \multicolumn{4}{|l|}{98674.00} \\
\hline \multicolumn{4}{|l|}{98682.20} \\
\hline 98697.01 & 98695.32 & 0.12 & $\{2,1,1\}<-1(11)$ \\
\hline 98699.46 & 98699.45 & 0.78 & $\{2,0,0\}<-1(10)$ \\
\hline $98710.82 *$ & 98712.11 & 0.09 & $\{2,0,0\}<--1(10)$ \\
\hline $98719.12 *$ & 98718.99 & 0.57 & $(0,0,1\}<-1(01)$ \\
\hline \multicolumn{4}{|l|}{98722.58} \\
\hline \multicolumn{4}{|l|}{98731.18} \\
\hline \multicolumn{4}{|l|}{98734.39} \\
\hline 98745.29 & 98743.23 & 0.40 & $\{1,0,0\}<-1(10)$ \\
\hline $98745.29 *$ & 98746.97 & 0.50 & $\{1,1,0\}<-0(00)$ \\
\hline 98748.55 & 98749.28 & 1.72 & $\{1,0,1\}<-1(01)$ \\
\hline $98758.65 *$ & 98755.06 & 1.18 & $\{2,0,1\}<-1(01)$ \\
\hline 98787.37 & & & \\
\hline
\end{tabular}




\begin{tabular}{|c|c|c|c|}
\hline $98810.59 *$ & 98810.45 & 0.20 & $\{0,0,0\}<-1(10)$ \\
\hline 98825.60 & 98826.66 & 0.15 & $\{1,0,0\}<-1(10)$ \\
\hline \multicolumn{4}{|l|}{98828.15} \\
\hline \multicolumn{4}{|l|}{98832.95} \\
\hline & 98839.84 & 0.23 & $\{2,0,1\}<-1(10)$ \\
\hline \multicolumn{4}{|l|}{98865.41} \\
\hline \multicolumn{4}{|l|}{98873.81} \\
\hline \multicolumn{4}{|l|}{98879.62} \\
\hline \multicolumn{4}{|c|}{$n=7$} \\
\hline 99432.58 & 99426.57 & 0.34 & $\{2,0,1\}<-1(01)$ \\
\hline \multicolumn{4}{|l|}{99489.52} \\
\hline 99500.03 & 99500.79 & 0.27 & $\{2,0,0\}<--1(10)$ \\
\hline 99503.93 & 99508.09 & 0.48 & $\{1,0,1\}<-1(01)$ \\
\hline 99503.93 & 99510.84 & 1.54 & $\{2,0,1\}<-1(01)$ \\
\hline 99512.44 & 99511.38 & 0.31 & $\{1,1,0\}<-0(00)$ \\
\hline 99526.35 & 99523.93 & 0.40 & $\{2,0,0\}<-1(10)$ \\
\hline 99549.26 & 99553.43 & 0.36 & $\{0,0,1\}<-1(01)$ \\
\hline 99559.47 & 99563.98 & 1.09 & $\{1,0,1\}<-1(01)$ \\
\hline \multicolumn{4}{|l|}{99566.53} \\
\hline 99569.53 & 99570.74 & 0.24 & $\{1,1,0\}<-0(00)$ \\
\hline 99573.28 & 99577.14 & 0.28 & $\{1,0,0\}<--1(10)$ \\
\hline 99578.69 & 99583.99 & 0.46 & $\{2,0,1\}<--1(01)$ \\
\hline 99620.92 & 99622.40 & 0.13 & $\{0,0,0\}<--1(10)$ \\
\hline \multicolumn{4}{|l|}{99626.92} \\
\hline \multicolumn{4}{|l|}{99634.52} \\
\hline \multicolumn{4}{|l|}{99637.13} \\
\hline 99641.53 & 99642.16 & 0.07 & $\{1,0,0\}<--1(10)$ \\
\hline
\end{tabular}




\begin{tabular}{|c|c|c|c|}
\hline \multicolumn{4}{|c|}{$\mathrm{n}=8$} \\
\hline 100017.42 & 100014.71 & 0.65 & $\{2,0,1\}<-1(01)$ \\
\hline \multicolumn{4}{|l|}{100021.62} \\
\hline 100056.14 & 100054.81 & 0.13 & $\{2,0,0\}<-1(10)$ \\
\hline \multicolumn{4}{|l|}{100058.60} \\
\hline 100059.95 & 100063.25 & 0.25 & $\{1,1,0\}<-0(00)$ \\
\hline 100069.96 & 100067.28 & 0.28 & $\{2,0,0\}<-1(10)$ \\
\hline 100069.96 & 100068.04 & 0.37 & $\{1,0,1\}<-1(01)$ \\
\hline 100069.96 & 100068.31 & 0.89 & $\{2,0,1\}<-1(01)$ \\
\hline \multicolumn{4}{|l|}{100076.91} \\
\hline \multicolumn{4}{|l|}{100082.21} \\
\hline & 100092.72 & 0.24 & $\{0,0,1\}<-1(01)$ \\
\hline 100097.98 & 100093.17 & 0.69 & $\{1,0,1\}<-1(01)$ \\
\hline \multirow[t]{2}{*}{100116.44} & 100117.19 & 0.21 & $\{1,0,0\}<-1(10)$ \\
\hline & 100123.49 & 0.15 & $\{2,0,1\}<-1(01)$ \\
\hline \multicolumn{4}{|l|}{100131.00} \\
\hline \multicolumn{4}{|l|}{100136.50} \\
\hline \multicolumn{4}{|l|}{100196.79} \\
\hline \multicolumn{4}{|c|}{$\mathrm{n}=9$} \\
\hline 100342.89 & 100337.41 & 0.09 & $\{2,0,0\}<-1(10)$ \\
\hline 100407.53 & 100407.70 & 0.84 & $\{2,0,1\}<-1(01)$ \\
\hline 100436.05 & 100437.46 & 0.20 & $(1,1,0)<-0(00)$ \\
\hline $100451.17^{*}$ & 100448.64 & 0.52 & $\{2,0,1\}<-1(01)$ \\
\hline \multirow[t]{4}{*}{100451.17} & 100451.49 & 0.37 & $\{1,0,1\}<-1(01)$ \\
\hline & 100453.93 & 0.17 & $\{0,0,1\}<-1(01)$ \\
\hline & 100454.32 & 0.19 & $\{2,0,0\}<-1(10)$ \\
\hline & 100457.81 & 0.25 & $\{1,0,1\}<-1(01)$ \\
\hline
\end{tabular}




\begin{tabular}{|c|c|c|c|}
\hline & 100461.26 & 0.17 & $(0,0,1)<-1(01)$ \\
\hline & 100486.79 & 0.17 & $\{1,0,0\}<-1(10)$ \\
\hline \multicolumn{4}{|c|}{$n=10$} \\
\hline \multicolumn{4}{|l|}{100648.25} \\
\hline \multicolumn{4}{|l|}{100663.41} \\
\hline & 100676.45 & 0.71 & $\{2,0,1\}<-1(01)$ \\
\hline \multicolumn{4}{|l|}{100693.03} \\
\hline & 100702.73 & 0.16 & $\{1,1,0\}<-0(00)$ \\
\hline $100711.79 *$ & 100712.27 & 0.25 & $\{2,0,1\}<-1(01)$ \\
\hline 100711.79 & 100713.91 & 0.58 & $\{1,0,1\}<-1(01)$ \\
\hline \multicolumn{4}{|l|}{100716.85} \\
\hline & 100724.21 & 0.12 & $\{0,0,1\}<-1(01)$ \\
\hline & 100729.69 & 0.17 & $\{2,0,0\}<-1(10)$ \\
\hline \multicolumn{4}{|l|}{100742.76} \\
\hline & 100750.72 & 0.14 & $\{1,0,0\}<--1(10)$ \\
\hline \multicolumn{4}{|c|}{$\mathrm{n}=11$} \\
\hline 100808.35 & 100811.56 & 0.07 & $\{2,0,0\}<--1(10)$ \\
\hline 100808.35 & 100811.56 & 0.17 & $\{2,0,1\}<-1(01)$ \\
\hline \multicolumn{4}{|l|}{100827.11} \\
\hline \multicolumn{4}{|l|}{100843.72} \\
\hline \multicolumn{4}{|l|}{100848.43} \\
\hline & 100858.34 & 0.24 & $\{2,0,1\}<-1(01)$ \\
\hline 100867.64 & 100871.84 & 0.23 & $\{2,0,1\}<-1(01)$ \\
\hline \multirow[t]{2}{*}{100897.41} & 100897.76 & 0.13 & $\{1,1,0\}<-0(00)$ \\
\hline & 100900.10 & 0.27 & $\{2,0,1\}<-1(01)$ \\
\hline $100907.36 *$ & 100907.31 & 0.46 & $\{1,0,1\}<-1(01)$ \\
\hline
\end{tabular}




\begin{tabular}{|l|l|l|l||}
\hline 100909.31 & \multicolumn{2}{|c|}{} & \\
\hline & 100918.36 & 0.09 & $\{0,0,1\}<-1(01)$ \\
\hline & 100932.15 & 0.14 & $\{2,0,0\}<-1(10)$ \\
\hline & 100945.60 & 0.11 & $\{1,0,0\}<-1(10)$ \\
\hline \multicolumn{5}{|c|}{$n=12$} & $\{2,0,1\}<-1(01)$ \\
\hline 100983.07 & 100983.02 & 0.11 & \\
\hline 100996.88 & & & \\
\hline 101009.49 & & & $\{2,0,1\}<-1(01)$ \\
\hline 101023.00 & 101020.80 & 0.39 & \\
\hline 101037.26 & & & $\{1,1,0\}<-0(00)$ \\
\hline 101042.91 & 101045.42 & 0.10 & $\{1,0,1\}<-1(01)$ \\
\hline $101053.72 *$ & 101053.15 & 0.40 & $\{2,0,1\}<-1(01)$ \\
\hline & 101058.88 & 0.10 & $\{2,0,0\}<-1(10)$ \\
\hline & 101062.01 & 0.05 & $\{0,0,1\}<-1(01)$ \\
\hline & 101065.78 & 0.07 & $\{2,0,0\}<-1(10)$ \\
\hline 101072.88 & 101076.66 & 0.07 & \\
\hline 101098.76 & & & \\
\hline
\end{tabular}




\begin{tabular}{|c|c|c|c|}
\hline \multicolumn{4}{|c|}{$\begin{array}{l}\text { Table 2: Observed and calculated photoionizaton line positions. } \\
\text { Lines marked }{ }^{*} \text { were used to optimize the quantum defects. } \\
\text { Lines thought to belong to other band systems have been omitted. }\end{array}$} \\
\hline $\begin{array}{l}\text { Experimental } \\
\text { line position }\left(\mathrm{cm}^{-1}\right)\end{array}$ & $\begin{array}{l}\text { Calculated } \\
\text { line position }\left(\mathrm{cm}^{-1}\right)\end{array}$ & $\begin{array}{l}\text { Calculated intensity } \\
\text { (a.u.) }\end{array}$ & $\begin{array}{l}\text { Assignment } \\
\left\{\mathrm{J}, \mathrm{p}^{\circ} \mathrm{p}^{*}\right\}<-\mathrm{J}\left(\mathrm{K}_{2} \mathrm{~K}_{\mathrm{c}}\right)\end{array}$ \\
\hline \multicolumn{4}{|c|}{$n=6$} \\
\hline $101799.62 *$ & 101797.65 & 0.17 & $\{0,0,0\}<-1(10)$ \\
\hline \multicolumn{4}{|l|}{101811.07} \\
\hline \multicolumn{4}{|l|}{101818.15} \\
\hline $101848.30^{*}$ & 101847.06 & 0.38 & $\{2,0,0\}<-1(10)$ \\
\hline $101855.26 *$ & 101856.14 & 0.76 & $\{1,0,1\}<-1(C 1)$ \\
\hline $101862.14^{*}$ & 101864.35 & 2.20 & $\{2,0,1\}<-1(01)$ \\
\hline 101864.62 & 101865.55 & 0.41 & $\{1,1,0\}<-0(00)$ \\
\hline \multicolumn{4}{|l|}{101870.46} \\
\hline $101914.71^{*}$ & 101914.73 & 0.70 & $\{2,0,0\}<-1(10)$ \\
\hline $101922.13^{*}$ & 101923.46 & 0.57 & $\{0,0,1\}<-1(u 1)$ \\
\hline $101929.44 *$ & 101930.57 & 0.16 & $\{2,0,0\}<-1(10)$ \\
\hline \multicolumn{4}{|l|}{101941.42} \\
\hline $101947.38 *$ & 101950.17 & 0.37 & $\{1,0,0\}<-1(10)$ \\
\hline $101959.28 *$ & 101959.68 & 0.45 & $\{1,1,0\}<-0(00)$ \\
\hline $101968.20 *$ & 101967.74 & 1.71 & $\{1,0,1\}<-1(01)$ \\
\hline $101970.27 *$ & 101969.31 & 1.03 & $\{2,0,1\}<-1(01)$ \\
\hline $102018.90 *$ & 102019.11 & 0.20 & $\{0,0,0\}<-1(10)$ \\
\hline \multicolumn{4}{|l|}{102100.71} \\
\hline \multicolumn{4}{|l|}{102039.69} \\
\hline & 102048.74 & 0.32 & $\{2,0,1\}<--1(01)$ \\
\hline & 102049.95 & 0.17 & $\{1,0,0\}<-1(10)$ \\
\hline
\end{tabular}




\begin{tabular}{|c|c|c|c|}
\hline \multicolumn{4}{|c|}{$n=7$} \\
\hline & 102651.64 & 0.42 & $\{2,0,1\}<--1(01)$ \\
\hline & 102662.23 & 0.11 & $\{0,0,0\}<-1(10)$ \\
\hline & 102718.12 & 0.22 & $\{2,0,0\}<-1(10)$ \\
\hline \multirow[t]{2}{*}{102721.05} & 102722.67 & 0.49 & $\{1,0,1\}<-1(01)$ \\
\hline & 102723.74 & 0.31 & $\{1,1,0\}<-0(00)$ \\
\hline 102726.68 & 102724.84 & 1.47 & $\{2,0,1\}<-1(01)$ \\
\hline 102735.79 & 107739.45 & 0.45 & $\{2,0,0\}<-1(10)$ \\
\hline 102758.64 & 102759.23 & 0.36 & $\{0,0,1\}<-1(01)$ \\
\hline \multirow[t]{2}{*}{102777.70} & 102779.09 & 1.07 & $\{1,0,1\}<\cdots 1(01)$ \\
\hline & 102784.11 & 0.23 & $\{1,1,0\}<-0(00)$ \\
\hline 102789.50 & 102785.69 & 0.27 & $\{1,0,0\}<-1(10)$ \\
\hline 102798.81 & 102797.77 & 0.42 & $\{2,0,1) \ll-1(01)$ \\
\hline 102830.29 & 102830.42 & 0.13 & $\{0,0,0\}<--1(10)$ \\
\hline \multicolumn{4}{|l|}{102859.81} \\
\hline \multicolumn{4}{|c|}{$n=8$} \\
\hline 103216.49 & 103218.16 & 0.07 & $\{0,0,0\}<-1(10)$ \\
\hline \multicolumn{4}{|l|}{103254.18} \\
\hline \multicolumn{4}{|l|}{103259.36} \\
\hline & 103228.49 & 0.67 & $\{2,0,1\}<-1(01)$ \\
\hline & 103267.55 & 0.10 & $(2,0,0) \ll-1(10)$ \\
\hline & 103274.01 & 0.25 & $\{1,1,0\}<\cdots 0(00)$ \\
\hline \multirow[t]{3}{*}{103278.61} & 103280.66 & 0.81 & $\{2,0,1\}<-1(01)$ \\
\hline & 103280.73 & 0.41 & $\{1,0,1\}<-1(01)$ \\
\hline & 103283.62 & 0.31 & $(2,0,0\}<-1(10)$ \\
\hline 103298.23 & 103299.26 & 0.24 & $(0,0,1)<-1(01)$ \\
\hline 103306.10 & 103306.65 & 0.65 & $(1,0,1)<-1(01)$ \\
\hline
\end{tabular}




\begin{tabular}{|c|c|c|c|}
\hline 103309.42 & & & \\
\hline \multirow[t]{2}{*}{103323.45} & 103326.76 & 0.20 & $\{1,0,0\}<-1(10)$ \\
\hline & 103335.37 & 0.15 & $\{2,0,1\}<-1(01)$ \\
\hline \multicolumn{4}{|l|}{103341.38} \\
\hline \multicolumn{4}{|c|}{$\mathrm{n}=9$} \\
\hline \multirow[t]{2}{*}{$103616.54 *$} & 103616.54 & 0.78 & $\{2,0,1\}<-1(01)$ \\
\hline & 103647.53 & 0.20 & $\{1,1,0\}<-0(00)$ \\
\hline $103661.51 *$ & 103659.65 & 0.56 & $\{1,0,1)<-1(01)$ \\
\hline \multirow[t]{3}{*}{103661.51} & 103660.75 & 0.36 & $\{2,0,1\}<-1(01)$ \\
\hline & 103668.16 & 0.20 & $\{2,0,0\}<-1(10)$ \\
\hline & 103668.25 & 0.17 & $(0,0,1\}<-1(01)$ \\
\hline $10 \geq 970.15$ & 103670.91 & 0.21 & $\{1,0,1\}<-1(01)$ \\
\hline \multicolumn{4}{|l|}{$1(3688.18$} \\
\hline 103695.07 & 103696.84 & 0.16 & $\{1,0,0\}<-1(10)$ \\
\hline \multicolumn{4}{|l|}{103701.42} \\
\hline \multicolumn{4}{|l|}{103707.90} \\
\hline \multicolumn{4}{|c|}{$\mathrm{n}=10$} \\
\hline \multicolumn{4}{|l|}{103838.84} \\
\hline \multicolumn{4}{|l|}{103860.17} \\
\hline \multicolumn{4}{|l|}{103869.51} \\
\hline \multicolumn{4}{|l|}{103878.56} \\
\hline $103886.66 *$ & 103884.27 & 0.66 & $\{2,0,1\}<-1(01)$ \\
\hline \multicolumn{4}{|l|}{103904.93} \\
\hline \multicolumn{4}{|l|}{103907.99} \\
\hline 103911.82 & 103912.73 & 0.15 & $\{1,1,0\}<-0(00)$ \\
\hline 103917.90 & & & \\
\hline
\end{tabular}




\begin{tabular}{|c|c|c|c|}
\hline & 103922.60 & 0.25 & $\{2,0,1)<--1(01)$ \\
\hline $103925.26 *$ & 103924.26 & 0.58 & $(1,0,1)<-1(01)$ \\
\hline \multicolumn{4}{|l|}{103926.77} \\
\hline \multirow[t]{3}{*}{103931.59} & 103931.48 & 0.12 & $\{0,0,1\}<-1(01)$ \\
\hline & 103941.39 & 0.16 & $\{2,0,0\}<-1(10)$ \\
\hline & 103960.84 & 0.13 & $\{1,0,0\}<-1(10)$ \\
\hline \multicolumn{4}{|c|}{$\mathrm{n}=11$} \\
\hline 103980.68 & 103983.05 & 0.13 & $\{1,0,1\}<-1(01)$ \\
\hline \multicolumn{4}{|l|}{103986.68} \\
\hline \multicolumn{4}{|l|}{103991.64} \\
\hline \multicolumn{4}{|l|}{103992.77} \\
\hline & 104019.64 & 0.18 & $\{2,0,1\}<-1(01)$ \\
\hline & 104068.60 & 0.19 & $\{2,0,1\}<-1(01)$ \\
\hline $104079.11 *$ & 104079.39 & 0.26 & $\{2,0,1\}<-1(01)$ \\
\hline \multicolumn{4}{|l|}{104102.21} \\
\hline & 104107.38 & 0.13 & $\{1,1,0\}<-0(00)$ \\
\hline 104107.99 & 104109.54 & 0.27 & $\{2,0,1\}<-1(01)$ \\
\hline $104113.88 *$ & 104117.42 & 0.47 & $\{1,0,1\}<-1(01)$ \\
\hline 104128.06 & & & \\
\hline
\end{tabular}




\begin{tabular}{|c|c|c|}
\hline Table 3: & $\begin{array}{l}\text { Linewidths for na } \\
\mathrm{H}_{2} \mathrm{O}^{+}(100) \text { (in } \mathrm{cm}\end{array}$ & erging on $\mathrm{H}_{2} \mathrm{O}^{+}(000)$ and \\
\hline Peak & $\begin{array}{l}\mathrm{H}_{2} \mathrm{O}^{+}(000) \\
{[\mathrm{MPI}]}\end{array}$ & $\begin{array}{l}\mathrm{H}_{2} \mathrm{O}^{+}(100) \\
\text { [Direct photoionization] }\end{array}$ \\
\hline $6 a$ & 1.3 & 2.8 \\
\hline $6 \mathrm{~b}$ & 1.3 & 1.3 \\
\hline $7 a$ & 1.0 & 3.0 \\
\hline $7 b$ & 1.8 & 2.1 \\
\hline $8 a$ & 3.5 & \\
\hline $8 b$ & 0.5 & -4.5 \\
\hline $9 a$ & [1.5] & 2.3 \\
\hline $9 \mathrm{~b}$ & 1.1 & 2.2 \\
\hline $10 \mathrm{a}$ & 0.7 & 1.5 \\
\hline $10 \mathrm{~b}$ & & 2.0 \\
\hline $11 a$ & 0.9 & 1.4 \\
\hline $11 \mathrm{~b}$ & & 1.4 \\
\hline $12 b$ & 0.6 & \\
\hline $13 b$ & 0.6 & \\
\hline
\end{tabular}




\begin{tabular}{|c|c|c|c|c|}
\hline State orbital & $\mu(000) n=3^{\prime \prime}$ & $\mu(000) n=6-12$ & $\mu(100) n=6-11$ & $\mu(100)^{0)}$ \\
\hline$D^{\prime \prime} B_{1} s$ & & [1.6274] & [1.6274] & \\
\hline$E^{\prime} B_{1} \quad d \sigma$ & 0.1243 & $0.1511(63)$ & $0.1423(11)$ & $0.155(5)$ \\
\hline$E^{\prime \prime} B_{2} \quad d \pi$ & 0.0771 & $0.0670(18)$ & $0.0704(13)$ & $0.067(7)$ \\
\hline$D^{\prime \prime 1} A_{2} \mathrm{~d} \pi^{+}$ & 0.3463 & $0.3607(548)$ & $0.3196(140)$ & $0.327(32)$ \\
\hline $\mathrm{F}^{\prime} \mathrm{B}_{1} \mathrm{~d} \delta^{+}$ & -0.0124 & $0.0086(20)$ & $0.0068(20)$ & $0.007(10)$ \\
\hline${ }^{1} A_{1} \quad d \delta$ & -0.0145 & $0.0179(47)$ & $-0.0008(35)$ & $0.013(9)$ \\
\hline $\begin{array}{l}\mathrm{s} / \mathrm{d \sigma} \\
\text { mixing angle }\end{array}$ & 0.0 & $0.1 \pi$ & $0.1 \pi$ & 0.0 \\
\hline $\mathrm{IP} / \mathrm{cm}^{-1}$ & 101773.0 & 101773.0 & 104983.0 & 104985.0 \\
\hline $\mathrm{Ry} / \mathrm{cm}^{-1}$ & 109733.8 & 109733.8 & 109733.8 & 109733.8 \\
\hline
\end{tabular}

a) Gilbert, Child and Johns ${ }^{(8)}$

b) Child and Jungen")

c) $\left|d \sigma^{\prime}\right\rangle=\cos \vartheta|d \sigma\rangle+\sin \vartheta|s\rangle$ 


\section{CHAPTER II}

\section{ULTRA-SENSITIVE DETECTION OF HYDROGEN MOLECULES}

BY (2+1) REMPI 


\begin{abstract}
Ultra-sensitive detection of molecular hydrogen is reported by using Doppler-free $(2+1)$ Resonance-Enhanced Multi-Photon Ionization (REMPI) through the E,F ${ }^{1} \Sigma_{B}^{+}$ state. By using an arrangement with two near transform-limited counterpropagating laser beams, a single shot detection efficiency of $6.810^{3} \mathrm{molecules} / \mathrm{cm}^{3}$ has been demonstrated. Frequency scans of the two-photon transitions show that the detection efficiency is limited by AC Stark effects.
\end{abstract}




\section{Introduction}

Over the last ten years laser-based detection schemes have enabled physical chemists to study in ever greater detail the dynamics of chemical reactions and photodissociation processes. Techniques such as Coherent Anti-Stokes Raman Scattering (CARS), Laser-Induced Fluorescence (LIF), degenerate four-wave mixing and Resonance-Enhanced Multi-Photon Ionization (REMPI) are the more common laser schemes in use today, with the latter clearly being the most sensitive technique.

State-specific detection of the hydrogen molecule has been of particular interest. There are a large number of studies which have explored the ro-vibrational product state distributions of the hydrogen molecule formed by photodissociation and from the reaction $\mathrm{D} / \mathrm{H}+\mathrm{H}_{2}-\rightarrow \mathrm{DH} / \mathrm{H}_{2}+\mathrm{H}$. State-specific detection of the molecular products of this reaction was carried out previously by Valentini and co-workers using CARS, and by Zare and co-workers using REMPI ${ }^{1)}$. The goal in both of these experiments was the determination of the final product state distributions. Our primary interest is in the (angle-resolved) differential cross-sections for specific final rovibrational states, which can be accessed by application of a crossed molecular beam technique ${ }^{2}$. These difficult experiments are possible provided that the detection efficiency for the hydrogen molecule can be improved with respect to the values that have been reported previously in the literature, namely approximately $1.710^{5}$ molecules/cc using (1+1) xuv+uv REMPI through the B- and C- state ${ }^{3)}$, and approximately $10^{6}$ molecules/cc for a (2+1) REMPI scheme through the E,F- state ${ }^{4}$. In the current chapter we demonstrate a single shot detection efficiency of approximately $6.810^{3}$ molecules/cc by using a Doppler-free $(2+1)$ REMPI scheme where the key is the use of two near-transform limited counterpropagating laser beams.

The use of Doppler-free two-photon resonant excitation schemes is a very popular technique in high-resolution spectroscopys), and a series of impressive results have been obtained with this method ${ }^{6}$. However, with a few exceptions ${ }^{7}$, the use of a Doppler-free two-photon technique with the aim of achieving superior detection efficiencies has hardly been explored. In studies of chemical reaction dynamics the 
molecules to be detected frequently travel over a wide range of laboratory velocities and angles, leading to an effective Doppler width which can significantly exceed the bandwidth of the lasers used for the detection. In our $\mathrm{D}+\mathrm{H}_{2}$ experiment, for example, the effective Doppler width for a typical ro-vibrational product state is approximately $3 \mathrm{~cm}^{-1}$. It is in these situations in particular, that application of a Doppler-free twophoton technique may be the preferred way to probe the number density of a particular ro-vibrational product state in a given volume element.

Two-photon excitation of $\mathrm{H}_{2}$ to the $\mathrm{E}, \mathrm{F}^{1} \boldsymbol{\Sigma}_{\mathrm{g}}^{+}$state was first demonstrated by Kligler and Rhodes ${ }^{8}$. The first rotational analysis for this transition was published by Marinero et.al.9) and later refined by Eyler et.al. ${ }^{10}$. For all isotopic combinations, detailed comparisons between the ab-initio transition moments and the relative experimental $(2+1)$ REMPI probabilities were published in a series of papers by Zare et.al. ${ }^{4.11}$. A number of groups have used two-photon excitation of $\mathrm{H}_{2}$ to the E,F ${ }^{1} \Sigma_{\mathrm{g}}^{+}$ state as a starting point for studies of $\mathrm{H}_{2}$ Rydberg states ${ }^{12.13}$.

The organization of this chapter is as follows. In Section 2, a theoretical comparison between $(1+1)$ REMPI and (2+1) REMPI of the hydrogen molecule will be presented, illustrating the relative merits of both techniques in particular situations. In Section 3, the experimental arrangement will be described and in Section 4 results for the detection of trace amounts of $\mathrm{DH}$ will be presented.

\section{Theory}

This section starts with a somewhat didactic treatment on how single-photon resonant and two-photon resonant absorption are described in perturbation theory, following the discussion of ref. 14. This will prove useful for making a detailed comparison of the relative merits of (1+1) REMPI and (2+1) REMPI. It will be demonstrated that in many situations of chemical interest the deciding factor in favor of the latter scheme is the fact that in $(2+1)$ REMPI a counterpropagating laser arrangement can be used in which the molecular Doppler width is eliminated from the problem. 
Perturbation theory of one- and two-photon transitions

In the presence of an electromagnetic field the molecular Hamiltonian is given as

$$
H=\sum_{1, y} \frac{1}{2 m_{1}}\left[\vec{p}-q \vec{A}\left(\vec{r}_{1}, t\right)\right]^{2}+V_{1 y}
$$

In perturbation theory this Hamiltonian is written in the form

$$
H=H^{0}+H^{\prime}
$$

where $\mathrm{H}^{\prime}$ is given, to a good approximation, by

$$
H^{\prime}=-\sum_{1} \frac{q}{m} \vec{A}(\vec{r}, t) \vec{p}_{1}
$$

The perturbation treatment consists of expressing the perturbed wavefunctions in terms of the unperturbed wavefunctions and solving the Schrodinger equation iteratively:

$$
\begin{aligned}
& \Psi_{g}(\vec{r}, t)=\sum_{g} \sum_{k} a_{k}^{(s)}(t) u_{k}^{0}(\vec{r}) e^{-1 \omega_{k} t} \\
& \frac{d}{d t} a_{k}^{(s+1)}=\frac{2 \pi}{i h} \sum_{n} H_{k n}(t) a_{n}^{(s)}(t) e^{-1 \omega_{k} t}
\end{aligned}
$$

where in the electric dipole approximation the matrix-elements $H_{k n}(t)$ are given as

$$
\begin{aligned}
H_{k n}(t) & =\frac{q}{m} \int u_{k}^{0}(\vec{r}) \vec{A}(\vec{r}, t) \cdot \vec{p}(\vec{r}, t) u_{n}(\vec{r}) d \tau_{r} \\
& =g A_{0} \omega_{k n}\langle k|\vec{\theta} \cdot \vec{r}| n\rangle
\end{aligned}
$$


where $\omega_{k n}=\omega_{k}-\omega_{n}$. When the perturbation calculation is carried out to first order, the solution of the wavefunctions looks as follows:

$$
\begin{aligned}
& \psi_{g}^{(1)}(\vec{r}, t)=\psi_{g}^{(0)}(\vec{r}, t)+ \\
& \sum_{k} \frac{2 \pi g A_{0}}{i h} \omega_{k g}[\vec{e}\langle g|\vec{r}| k\rangle] u_{k}^{0}(\vec{r}) \frac{\exp \left[i\left(\omega_{k g}-\omega\right) t\right]}{\omega_{k g}-\omega}
\end{aligned}
$$

This equation describes the time evolution from the initial ground state $|\mathrm{g}\rangle$ to other states $|k\rangle$. Populations in the excited states $|k\rangle$ are found as the squares of the timedependent coefficients of the wavefunctions $u_{k}{ }^{\circ}(r)$ in Equation [6] and stay small unless the laser frequency $\omega$ is close to the transition frequency $\omega_{\mathrm{kg}}$. In order to analyze the transition rate between the states $|\mathrm{g}\rangle$ and $|\mathrm{k}\rangle$ precisely it has to be taken into account that neither the laser nor the molecule have a precise frequency, but rather that there are distributions $g_{M}(\omega)$ and $g_{L}(\omega)$ where:

$$
\int g_{M}(\omega) d \omega-\int g_{L}(\omega) d \omega=1
$$

This leads to the following equation for the time-evolution of the excited state population

$$
\begin{aligned}
\frac{N_{k}(t)}{N_{g}(t)} & -\frac{4 \pi^{2} g^{2} A_{0}^{2}}{h^{2}}|\vec{e} \cdot(g|\vec{I}| k)|^{2} \quad * \\
& \int_{0} \int_{0}^{\frac{4}{g^{2} n^{2}\left(\omega_{k g}-\omega\right) t}} \frac{t}{\left(\omega_{k g}-\omega\right)^{2}} g_{M}\left(\omega_{k g}\right) g_{L}(\omega) \omega_{k g}^{2} d \omega_{k g} d \omega
\end{aligned}
$$


After making a Jacobian transformation from $\left(\omega_{\mathrm{kg}}, \omega\right)$ to $\left(\omega_{\mathrm{av}}=\left[\omega_{\mathrm{kg}}+\omega\right] / 2, \delta=\left[\omega_{\mathrm{kg}}-\right.\right.$ $\omega] / 2$ ) and evaluating the integral over $\delta$ Equation [8] can be rewritten as

$$
\frac{N_{k}(t)}{N_{g}(t)}=\frac{32 \pi^{3} g^{2} A_{0}^{2} t}{h^{2}}|\vec{\theta} \cdot\langle g|\vec{r}| k)|^{2} \int_{0}^{\infty} g_{M}\left(\omega_{a v}\right) g_{L}\left(\omega_{a v}\right) \omega_{a v}^{2} d \omega_{a v}
$$

The integral on the righthand side of this equation shows that two rather different situations can occur depending on the relative widths of the frequency distributions of the laser and the molecule. If the molecular linewidth is much narrower than the laser linewidth, then $g_{M}(\omega)$ will be a much sharper function than $g_{L}(\omega)$, and consequently the integral can be approximated by $\omega^{2} \mathrm{~g}_{\mathrm{L}}(\omega)$, indicating that the laser linewidth, rather than the molecular linewidth, puts a limit on the uppumping rate. On the other hand, if the laser linewidth is much narrower than the molecular linewidth, the integral can be approximated by $\omega^{2} g_{M}(\omega)$ and apart from the photonflux of the laser,

$$
F=\frac{\pi \omega A_{0}^{2}}{\mu_{0} h c}
$$

the uppumping rate is uniquely determined by the absorption cross section of the molecule, which can be written as

$$
\sigma_{g k}-64 \pi^{2} \alpha \omega g_{M}(\omega)|(g|\vec{r}| k\rangle|^{2}
$$

where $\alpha$ is the fine-structure constant.

In order to understand the occurrence of two-photon resonances, the perturbation treatment has to be carried on to second order, with the evaluation of the coefficients $a_{k}^{(2)}(t)$. When this procedure is carried out, the following solution is found, 


$$
\begin{aligned}
& \Psi_{g}^{(2)}(\vec{r}, t)=\psi_{g}^{(1)}(\vec{r}, t) \\
& +\frac{4 \pi^{2} g^{2} A_{0}^{2} \omega^{2}}{h^{2}} \sum_{f} S_{g f} u_{f}^{0}(\vec{r}) \frac{1-\exp \left[-i\left(\omega_{f g}-2 \omega\right) t\right]}{\omega_{f g}-2 \omega} \\
& (+ \text { otherterms }) \\
& \text { where } S_{g f}-\sum_{k} \frac{(\vec{e} \cdot\langle g|\vec{r}| k\rangle)(\langle k|\vec{r}| f\rangle \cdot \vec{e})}{\omega_{k g}-\omega}
\end{aligned}
$$

where, in this case, the coefficients $a_{k}^{(2)}(t)$ stay small unless the molecular frequency $\omega_{\mathrm{fg}}$ is close to $2 \omega$. Instead of a single transition moment between the initial state $|\mathrm{g}\rangle$ and final state $|f\rangle$, a term $S_{g r}$ appears in this equation which consists of a sum of products of integrals connecting the ground state $|\mathrm{g}\rangle$ to an intermediate state $|\mathrm{k}\rangle$, and the intermediate state $|k\rangle$ to final state $|f\rangle$. The contribution to the state sum from a particular intermediate state $|\mathrm{k}\rangle$ can be enhanced if the laser frequency $\omega$ is close to the transition frequency $\omega_{\mathrm{kg}}$, and thus two-photon absorption can be very efficient if a two-color scheme is used. For detection of the DH molecule this option does not exist since the intermediate states that dominate the two-photon transition moment require excitation in the extreme ultra-violet. Also, in the current experiment a one-color two-photon resonant technique will be seen to provide some major advantages.

Following a similar treatment as for the one-photon case, the two-photon transition rate may be calculated as

$$
\begin{aligned}
W_{g f}^{(2)} & =\frac{1}{N_{g}}\left(\frac{d}{d t}\right) N_{f} \\
& =128 \pi^{5} \frac{\sigma^{4} A_{0}^{4}}{h^{4}} \iint\left|s_{g t}\right|^{2} \omega_{a v}^{4} g_{M}\left(2 \omega_{a v}\right) g_{L}\left(\omega_{a v}\right) d \omega_{a v}
\end{aligned}
$$

A dependence on the linewidth of the laser and the linewidth of the molecular transition similar to the one-photon case, can be recognized. Rewriting Equation [13] in terms of the laser power I and performing the integral over $\omega_{2 v}$ leads to the following convenient equations for the two-photon absorption cross section and the 
effective two-photon absorption rate

$$
\begin{aligned}
\boldsymbol{\sigma}_{g f}^{(2)} & =\alpha I \\
& =\frac{(2 \pi)^{4}}{h c^{2}} \omega\left|M_{g f}\right|^{2} g_{\text {offoctIve }}(\omega) I
\end{aligned}
$$

and

$$
\begin{aligned}
W_{g f}^{(2)} & =0 \frac{2 \pi I}{h \omega} \\
& =\frac{(2 \pi)^{5}}{h^{2} c^{2}}\left|M_{g f}\right|^{2} g_{\text {effoctive }}(\omega) I^{2}
\end{aligned}
$$

where the two-photon transition moment $\mathrm{M}_{\mathrm{gf}}$ has been introduced as

$$
\left|M_{g f}\right|^{2}=\left.1 \quad \frac{\sum_{\alpha}}{k} \frac{(\vec{e} \cdot\langle g|\vec{\mu}| k))(\langle k|\vec{\mu}| f\rangle \cdot \vec{e})}{E_{k g}-\frac{h \omega}{2 \pi}}\right|^{2}
$$

and the integral over the lineshape distributions has been replaced by an effective lineshape geffective $(\omega)$. Unlike the one-photon case, the two-photon absorption cross section is proportional to the laser power, in agreement with the fact that the twophoton absorption rate has a quadratic dependence on laser power.

The application of a (2+1) REMPI technique opens up the possibility of setting up a counterpropagating laser arrangement in which the Doppler shifts with respect to the two laser directions cancel and the first order Doppler shift drops out of the resonance condition:

$$
\omega_{g f}=\omega\left\{\left[1+\frac{u_{x}}{c}\right]_{\text {laser I }}+\left[1-\frac{u_{x}}{c}\right]_{\text {leser II }}\right\}=2 \omega
$$

Thus, in the absence of additional line broadening mechanisms, the molecular linewidth $g_{M}(\omega)$ reduces from the Doppler width to the natural linewidth, and when the laser frequency is tuned to satisfy the resonance condition, all molecules will be able to absorb two photons, one from each laser beam, independent of their velocity vector. 
The lifetimes of the states that are accessed in the two-photon process are about 100 nsec $^{15)}$, and thus the natural linewidth is considerably narrower than the linewidths which are achievable in most high power pulsed laser systems. Therefore, if there are no other line broadening mechanisms, the effective linewidth function geffective $(\omega)$ in Equations [5] and [6] becomes equivalent to the laser linewidth function $g_{L}(\omega)$, and it will prove advantageous to use a narrowband laser in this detection scheme. This should come as no surprise, since the probability of finding a 'matching photon' in the second, counterpropagating laser is inversely proportional to the bandwidth of the laser.

Comparison of the detection efficiencies for molecular hydrogen using (1+1) REMPI and $(2+1)$ REMPI

The most precise quantitative work on the efficiency of hydrogen detection by $(1+1)$ Xuv+uv REMPI through the B- or C-state was published a few years ago by Kung et.al. ${ }^{3)}$ Using frequency-tripling of the doubled output of a $0.3 \mathrm{~cm}^{-1}$ linewidth pulsed dye laser, these authors report a noise-limited detection limit of $1.710^{5} \mathrm{H}_{2}$ molecules/cc in a given quantum state over an integration period of 100 lasershots, corresponding to a single shot detection limit of $1.710^{6}$ molecules/cc. In this experiment, the laser linewidth was $0.8 \mathrm{~cm}^{-1}(24 \mathrm{GHz}$ ) at the extreme ultraviolet (xuv) transition frequency, which is much larger than the molecular linewidth, which is a combination of the natural linewidth (approximately $250 \mathrm{MHz}$ ) and the residual Dopplerwidth of the molecular beam used. Thus it follows from Equation [9] that it is possible to increase the excitation rate (and thus the detection sensitivity) by nearly two orders of magnitude when the relatively broad bandwidth laser of Kung et.al. is replaced by a more narrowband $x u v$ laser. In fact, Cromwell reports a detection efficiency of $\approx 10^{4}$ molecules/cc for molecules with a Dopplershift within the bandwidth of a near transform-limited $210 \mathrm{MHz}$ FWHM xuv laser ${ }^{16)}$.

However, here a major problem with the $(1+1)$ technique is encountered. The high detection sensitivity achieved with the near-transform limited laser is only 
available as long as the molecular linewidth is smaller than or roughly equal to the laser linewidth. If there is a mechanism that broadens the molecular linewidth beyond the laser linewidth, Equation [9] shows that the narrow linewidth of the laser ceases to benefit the detection efficiency, which instead becomes inversely proportional to the linewidth of the molecular transition. In the experiments on the hydrogen exchange reaction which we carry out in our laboratory, the typical effective Doppler width of the hydrogen product molecules is about $3 \mathrm{~cm}^{-1}$. Using the higher value for the detection efficiency reported by Cromwell it follows that in this case the detection sensitivity would drop to about $4.310^{6}$ molecules/cc for the $(1+1)$ REMPI technique. It should be pointed out that the hydrogen exchange reaction is by no means an exceptional case. In many situations of chemical interest, large effective Doppler widths are encountered, where the detection efficiency using $(1+1)$ REMPI will drop to $10^{6}-10^{7}$ molecules/cc.

In order to calculate the efficiency of the two-photon process, Equation [15] describing the rate of the two-photon absorption is brought into the following form:

$$
W_{\sigma t}^{(2)}\left[\sec ^{-1}\right] \sim 1.210^{-9} *\left|M_{g t}\right|^{2}[a . u .] * I_{1 a s e r}^{2}\left[\frac{W^{2}}{c m^{4}}\right]
$$

where it has been assumed that a counterpropagating laser beam arrangement exists with two beams of equal laser power $I_{\text {Lerer }}$ Furthermore, reflecting conditions existing in our experiment, a $150 \mathrm{MHz}$ laser bandwidth was used to obtain Equation [18]. The transition moments for the hydrogen two-photon excitations used in this scheme have been determined in an ab-initio quantum mechanical calculation and have been compared experimentally with results obtained for known ro-vibrational state populations. For DH Q-branch excitations E,F ${ }^{1} \Sigma_{g}^{+}\left(v^{\prime}=0, J^{\prime}=J^{\prime \prime}\right)<--X^{1} \Sigma_{g}^{+}\left(v^{\prime \prime}=0, J^{\prime}\right)$, the squared transition moments are found to be approximately 7 atomic units, whereas for excitations DH E, $F^{1} \Sigma_{8}^{+}\left(v^{\prime}=1, J^{\prime}=J^{\prime \prime}\right)<-X^{1} \Sigma_{z}^{+}\left(v^{\prime \prime}=1, J^{\prime \prime}\right)$, the squared transition moments are approximately 24 atomic units ${ }^{4}$. Although not constant, the transition moments are relatively insensitive to the choice of the rotational quantum number. 
The application of $(2+1)$ REMPI detection requires the generation of laser light around $200 \mathrm{~nm}$. As discussed in the next section, it is possible to generate several $\mathrm{mJ} /$ pulse at these wavelengths. An estimate of the uppumping rate can be obtained by assuming a power of about $0.5 \mathrm{~mJ} /$ pulse for both counterpropagating laser beams and an experimental $201 \mathrm{~nm}$ pulse length of about $7 \mathrm{nsec}$. If the laser beams are focussed to a waist of $300 \mu \mathrm{m}$, then the laser power will be $10^{8} \mathrm{~W} / \mathrm{cm}^{2}$ and the two-photon uppumping rate for $\mathrm{H}_{2}\left(\mathrm{v}^{\prime \prime}=0\right)$ is found to be about $8.410^{7} \mathrm{sec}^{-1}$. The significance of this result can be understood if the rate equation for depopulation of the ground state is considered. Assuming a typical photoionization cross section of $10^{-17} \mathrm{~cm}^{2}$, the rate of the single-photon ionization step is about $10^{9} \mathrm{sec}^{-1}$ at the laser power given above, and therefore it will be assumed that after the initial excitation the molecule is immediately ionized and there are no two-photon transitions from the excited state to the ground state. The depopulation of the ground state is then given by

$$
\frac{d N_{g}}{d t}=-W_{g I}^{(2)} N_{g}
$$

so after the laser pulse is over

$$
\begin{aligned}
N_{g} & =N_{g, 0} \exp \left[-W_{g f}^{(2)} \Delta T_{\text {lager }}\right] \\
N_{\text {lan }}^{+} & =N_{g, 0}\left(1-\exp \left[-W_{g f}^{(2)} \Delta T_{\text {laser }}\right]\right)
\end{aligned}
$$

and we find that in this situation about $45 \%$ of the molecules which were initially in the ground state are ionized.

The calculation given above can be related to the detection efficiency. Similar to the case of $(1+1)$ REMPI, it will be assumed that the detection limit for the experiment lies at the point where in a single lasershot a signal-to-noise ratio of unity can be achieved. This means that in a background-free experiment we need to detect on average one ion per lasershot. In the current experiment, detection of ions is possible over a length of $2.5 \mathrm{~cm}$ along the laseraxis. This means that when the laser is 
focussed to a $300 \mu \mathrm{m}$ spot, as described above, the size of the effective detection volume will be $1.8 \mathrm{~mm}^{3}$. Using the result of the calculation that on average $2.3 \mathrm{DH}$ molecules are required in the detection volume in order to detect a single ion, this would lead to a detection sensitivity of approximately 1300 molecules $/ \mathrm{cm}^{3}$, which, in addition to the fact that it does not require the molecules to have a particular Doppler shift, is almost one order of magnitude better than the $(1+1)$ REMPI detection efficiency reported by Cromwell.

The calculated dependence of the ion signal on the size of the detection volume is shown in Figure 1 for two values of the laser power in both counterpropagating laser beams. In Figure 1, the product of the fraction of molecules ionized $\left(\mathrm{N}_{\text {ior }}^{+} / \mathrm{N}_{\mathrm{g}, 0}\right)$ and the size of the detection volume $\left(\mathrm{cm}^{3}\right)$ is calculated. The product of this number and the molecular number density $\left(\mathrm{cm}^{-3}\right)$ gives the number of ions formed per lasershot. It is observed that for spotsizes below $200 \mu \mathrm{m}$, the two-photon transition is readily saturated at both laser powers, and the signal shows a quadratic dependence on the diameter of the detection volume. For a laser power of $0.5 \mathrm{~mJ} / \mathrm{pulse}$, the optimum efficiency is achieved for a waist of about $250 \mu \mathrm{m}$, whereas for a laser power of 1.0 $\mathrm{mJ} /$ pulse the optimum efficiency occurs around a waist of $380 \mu \mathrm{m}$. The theoretical detection efficiencies at these waists are $1.110^{3}$ molecules $/ \mathrm{cm}^{3}$ and $5.710^{2}$ molecules $/ \mathrm{cm}^{3}$, respectively. As the waist is increased further, a situation is reached where the detection efficiency falls off quadratically with the diameter of the detection volume.

It should be stressed that the calculations given above assume that experimentally there are no mechanisms which broaden the bandwidth of the twophoton transition beyond the bandwidth of the laser. As our experimental results will show, the $(2+1)$ REMPI detection efficiency is in fact reduced by the occurrence of a dynamic Stark effect. 


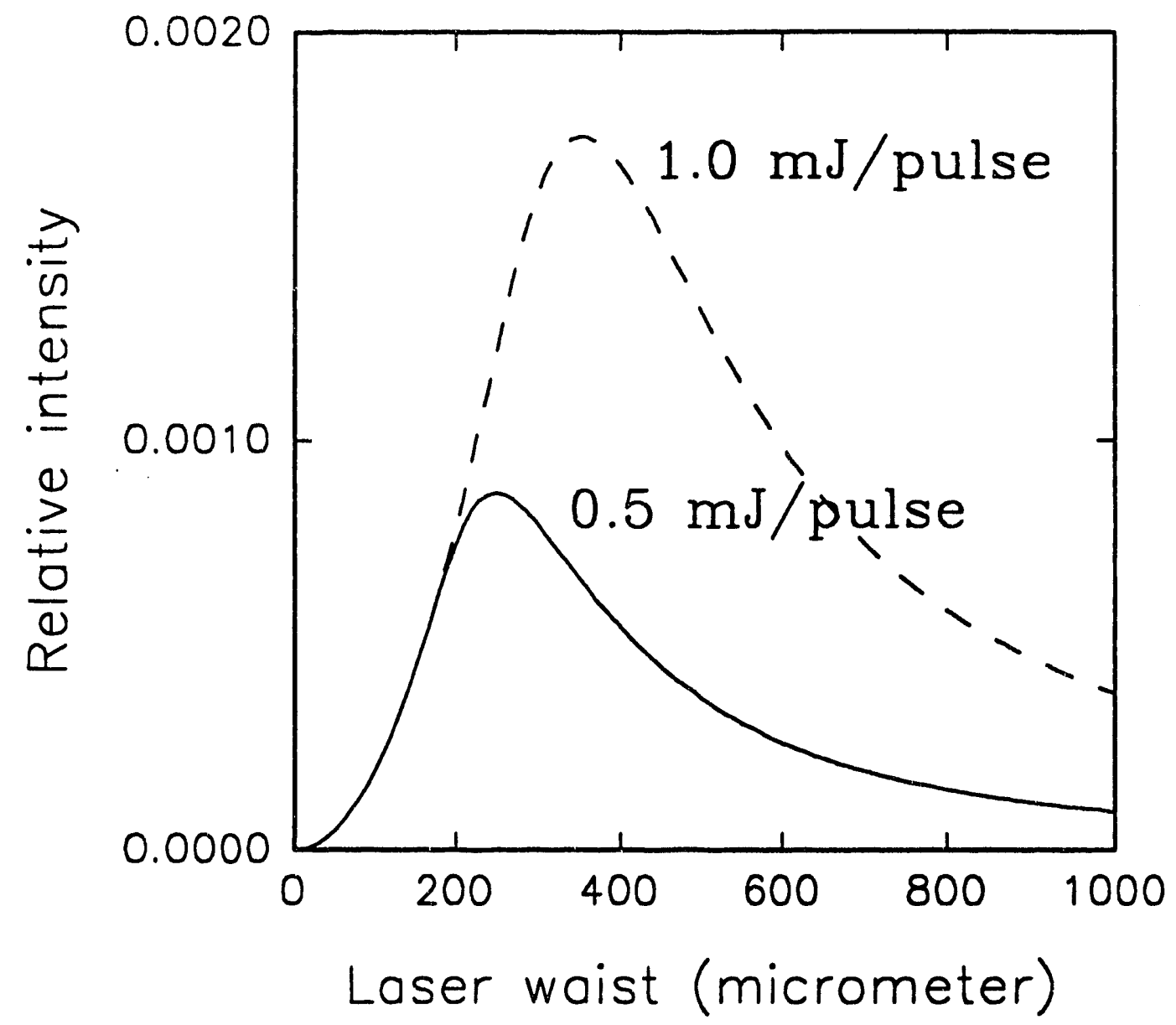

Figure 1 The dependence of the detection sensitivity on the size of the laser focus. The number plotted on the left is the product of two-photon transition probability and the size of the detection volume $\left(\mathrm{cm}^{3}\right)$. See text for details. 


\section{Experimental}

The experimental set-up used in these measurements consists of a vacuum chamber in which two counterpropagating tunable ultra-violet laser beams are overlapped and focussed to a waist of several hundred $\mu \mathrm{m}$. Ions produced by the lasers are accelerated and mass-selected by time-of-flight prior to detection using a position-sensitive ion detector. A detailed description will now be given of the laser system used and various elements in the experimental arrangement which are necessary to obtain the high sensitivity for the detection of hydrogen molecules.

The near transform-limited tunable laser system used in this experiment is based on pulse-amplification of the continuous output of a Coherent 699-29 Autoscan ringlaser in four stages using three prism dye amplifiers. In this amplifier chain, which is pumped by a $10 \mathrm{~Hz}$ Quantel Model YG-592 Nd:YAG laser, the second dye cell is double-passed. This laser is capable of delivering approximately $100 \mathrm{~mJ}$ of visible laser light in a $12 \mathrm{nsec}$ pulse, with a near transform-limited bandwidth which was recently re-measured to be approximately $52 \mathrm{MHz}$. The detailed design of this laser system was published previously ${ }^{17}$.

In order to improve the spatial mode quality of the laser, the expanding telescope between the first $(1 \mathrm{~mm})$ and second $(3 \mathrm{~mm})$ dye amplifier was set up in such a way that the laser converged to a $2 \mathrm{~mm}$ spot at the entrance to the beamexpanding telescope between the second and third $(6 \mathrm{~mm})$ amplifier, where the beam size was increased by a factor three, rather than by a factor two, as in the design of Cromwell et.al. The use of a converging laser beam through the second dye amplifier reduces the appearance of diffraction rings, in particular on the second pass through this cell, giving a beam which can be amplified more homogeneously in the final amplifier, without any loss of power.

The visible output was doubled in KDP using an Inrad Autotracker II and separated from the resulting ultraviolet by using a dielectric mirror coated for $300 \mathrm{~nm}$. The polarization of the residual visible light was rotated through $90^{\circ}$ by using a double Fresnel rhomb and the visible and ultraviolet beams were recombined using another 
dielectric mirror coated for $300 \mathrm{~nm}$. An $8 \times 8 \times 8 \mathrm{~mm}^{3}$ BBO crystal cut at $74^{0}$ was used to generate the tunable light around $201 \mathrm{~nm}$, where laser powers up to $4.5 \mathrm{~mJ} / \mathrm{pulse}$ were observed.

In order to focus the laser beam down to a waist of several hundred $\mu \mathrm{m}, \mathrm{a}$ telescope was used consisting of two $300 \mathrm{~mm}$ lenses which were set up to give a focus approximately $5 \mathrm{~m}$ downstream. For an ideal $6 \mathrm{~mm}$ Gaussian beam the size of the waist would be $60 \mu \mathrm{m}$. It was not expected that our laser would approach this value, and by applying a knife edge technique ${ }^{18}$, the spotsize inside the vacuum chamber was in fact experimentally determined to be about $500 \mu \mathrm{m}$. At some wavelengths, for example near the $a^{\prime \prime} \Sigma_{g}^{+}<-X^{1} \Sigma_{g}^{+}$two photon transition in the $N_{2}$ molecule ${ }^{19)}$, a spark was observed at the focus between the two $300 \mathrm{~mm}$ lenses. To alleviate this problem the first $300 \mathrm{~mm}$ lens will soon be replaced by a $200 \mathrm{~mm}$ negative focal length lens. At the same time this change is expected to reduce the focal spotsize by about $30 \%$, which might improve the detection efficiency, as can be seen from Figure 1.

After the telescope the $200 \mathrm{~nm}$ light was split into two beams of approximately equal power by using a 50/50 beamsplitter. The two laser beams were directed to the vacuum chamber using dielectric mirrors coated for $207 \mathrm{~nm}$ and $193 \mathrm{~nm}$. The $207 \mathrm{~nm}$ mirrors were found to be preferable at angles of incidence of $45^{\circ}$ and larger, whereas the $193 \mathrm{~nm}$ mirrors provided better reflectivity at points along the beamline where slightly smaller angles of incidence were required. Care was taken to avoid excessive transmission losses at any of the turning mirrors. Available $1 \mathrm{~cm}$ quartz prisms proved to give significant absorption and reflection losses.

The two vertically polarized counterpropagating lasers entered and exited the vacuum chamber through $5 \mathrm{~mm}$ thick Heraeus Amersil Suprasil CFQ Standard SVIA windows placed at Brewster's angle. In view of the high power density of the $201 \mathrm{~nm}$ beams, the windows were placed $1 \mathrm{~m}$ from the focus inside the vacuum chamber. In this configuration, the lasers could be operated for extended periods of time (approximately 100 hours) before any significant damage occurred. The windows could be rotated while the machine remained under vacuum. 
The counterpropagating laser beams entered the central portion of the chamber after passing through a lightbaffle consisting of three skimmers made of anodized aluminum. While the first two skimmers had a $2 \mathrm{~mm}$ aperture, it was found that the aperture of the third skimmer needed to be increased to about $4 \mathrm{~mm}$ to avoid producing scattered light from the beam exiting the chamber on that particular side. Electrodes were place behind the third skimmer in order to inhibit the acceleration of spurious photoelectrons towards the ion extraction region, but operation of the electrodes was not found to be necessary.

At the detector, the power density of the counterpropagating beams is approximately $10^{8} \mathrm{~W} / \mathrm{cm}^{2}$, which is sufficient to non-resonantly dissociate and/or ionize a large number of background molecules within the duration of the laser pulse. Therefore it is crucial to apply mass selection to the ionized products. The ions were accelerated to a kinetic energy of approximately $1.5 \mathrm{kV}$ over a $1 \mathrm{~cm}$ acceleration length. After entering a drifttube through a $90 \%$ transmitting stainless steel mesh, they reached the detector following a $13.5 \mathrm{~cm}$ long drift length. The detector used in these experiments was a position-sensitive ion detector consisting of a $32 \mathrm{~mm}$ diameter dual microchannel plate amplifier (Hamamatsu F2224-21PFFX) followed by a P-20 phosphor screen held at $+3 \mathrm{kV}$, and a fiberoptic coupler. The microchannel plates, which have a quantum efficiency of approximately $60 \%$, were operated by applying a 50 nsec long pulse of up to $1.8 \mathrm{kV}$ to the anode surface, with the cathode surface being held at ground throughout. Thus the microchannel plate gain was only present during the arrival of the ion mass selected by the choice of the delaytime between the high voltage pulse for the microchannel plate and the Nd:YAG Q-switch pulse.

At the initial stage of these experiments, it was found that a substantial spurious signal arose from the formation of photoelectrons inside the field-free drifttube. These photoelectrons were formed by the laser and remained detectable with a 1/e decaytime of approximately $500 \mathrm{nsec}$. In order to eliminate this signal, the aforementioned light baffles were introduced, and, more importantly, the drifttube was held at a positive bias with respect to the microchannel plate cathode, thereby completely eliminating this problem. Somewhat surprisingly, it was found that a bias 
of as much as $+60 \mathrm{~V}$ was required to inhibit the detection of these photoelectrons. This would seem to indicate that the cathode acquires a slightly positive voltage during the pulsing of the microchannel plates. After prolonged, successful operation of the microchannel plates some arcing problems were encountered between the microchannel plate anode and the phosphor screen. It is believed that this may be a side-effect of the pulsed operation of the microchannel plates.

The photon signal at the phosphor screen was imaged onto a Hamamatsu C3077 video camera by using a 4.3:1 demagnifying telescope. The video camera was interfaced to a Hamamatsu DVS 3000 image processor. At a clockrate of approximately $30 \mathrm{~Hz}$ video images are transferred and digitized. A discriminator level serves to filter out the thermal and/or readout noise associated with the operation of the camera. This ensures that there is no instrument-related lower limit on the number of ions that is required for a detectable signal ${ }^{20}$. In the experiments reported in this chapter, spectra were recorded by transferring video images accumulated on the image processor to an IBM PC, where the total signal intensity was calculated and stored.

In order to measure the detection efficiency of the $(2+1)$ REMPI scheme, $\mathrm{H}_{2}$ gas was admitted to the vacuum chamber until a change of $510^{-7}$ Torr was observed on the Granville Phillips Bayard-Alpert ionization gauge. Under these conditions it was observed that with the detection laser $30 \mathrm{~cm}^{-1}$ detuned from the DH E, $F(v=0, J=0)$ $<-\mathrm{X}(\mathrm{v}=0, \mathrm{~J}=0)$ resonance, the background at the $\mathrm{DH}^{+}$arrival time was less than one count per hundred lasershots. Consequently the single shot detection efficiency corresponds to the $\mathrm{DH}$ density where on average one $\mathrm{DH}^{+}$ion is formed per lasershot.

The power dependence of the (2+1) REMPI process was determined by recording the $(2+1)$ REMPI spectrum of $\mathrm{H}_{2}(\mathrm{~J}=1)$ at a number of laser powers. The power of the $201 \mathrm{~nm}$ light was attenuated by turning down the voltage of the flashlamps in the Quantel YG 592 Nd:YAG laser which pumps the amplifier chain. This method of attentuation was chosen in order to preserve the mode and the alignment of the $201 \mathrm{~nm}$ beam as much as possible. Since the saturation of the dye amplifiers depends on the power of the Nd:YAG laser, some change in the mode of the $201 \mathrm{~nm}$ light is anticipated, however. 


\section{Results}

The power dependence of $(2+1)$ REMPI of $\mathrm{H}_{2}(\mathrm{~J}=1)$

In Figure 2 the $(2+1)$ REMPI spectrum of $\mathrm{H}_{2}(\mathrm{v}=0, \mathrm{~J}=1)$ is shown for three different laser powers. The laser power indicated in Figure 2 was measured between the first and the second $300 \mathrm{~mm}$ lens of the telescope which sets up the focussing of the counterpropagating beams. Consequently, this number is to be interpreted as a relative measure of the power density inside the vacuum chamber.

The power dependence of the $(2+1)$ REMPI detection of $\mathrm{H}_{2}(\mathrm{v}=0, \mathrm{~J}=1)$ is shown in Figure 3, where the signal intensity measured at the peak of the frequency spectrum is plotted versus the laser power. The power dependence follows a near-quadratic behaviour in the energy range from 1.5 to $3.2 \mathrm{~mJ} / \mathrm{pulse}$, whereas for energies below $1.5 \mathrm{~mJ} / \mathrm{pulse}$ the signal drops off more rapidly. This is due to the fact that at lower energies the ionization step is not yet saturated. Figure 3 does not show any pronounced leveling-off of the power dependence towards higher pulse energies. We have on occasion seen preliminary evidence of this kind of behaviour, which would be indicative of the onset of saturation of the two-photon excitation step. In Figure 3 we have chosen to display a power dependence measurement which was carried out under the same conditions as existed during our calibration of the $\mathrm{DH}(\mathrm{v}=0, \mathrm{~J}=0)$ detection efficiency, to be discussed below.

The spectra in Figure 2 show evidence of an AC Stark effect. As the laser power is increased both a shift and a broadening of the line profile is observed. The occurrence of an AC Stark effect is inherent in the use of a two-photon technique, since the same matrix elements which couple the ground and two-photon excited state to the virtual intermediate state, shift these states with respect to the intermediate state $^{5,21}$. Assuming a homogeneous laser spatial profile, one can calculate that with a total power of $1.2 \mathrm{~mJ}$ focussed to a $500 \mu \mathrm{m}$ spotsize, the observed shifts of the twophoton transitions in Figure 2 are about $15 \mathrm{MHz}$ per $\mathrm{MW} / \mathrm{cm}^{2}$. This value agrees reasonably well with a value of 3-6 $\mathrm{MHz}$ per $\mathrm{MW} / \mathrm{cm}^{2}$ estimated by Hessler ${ }^{22}$. Rhodes 


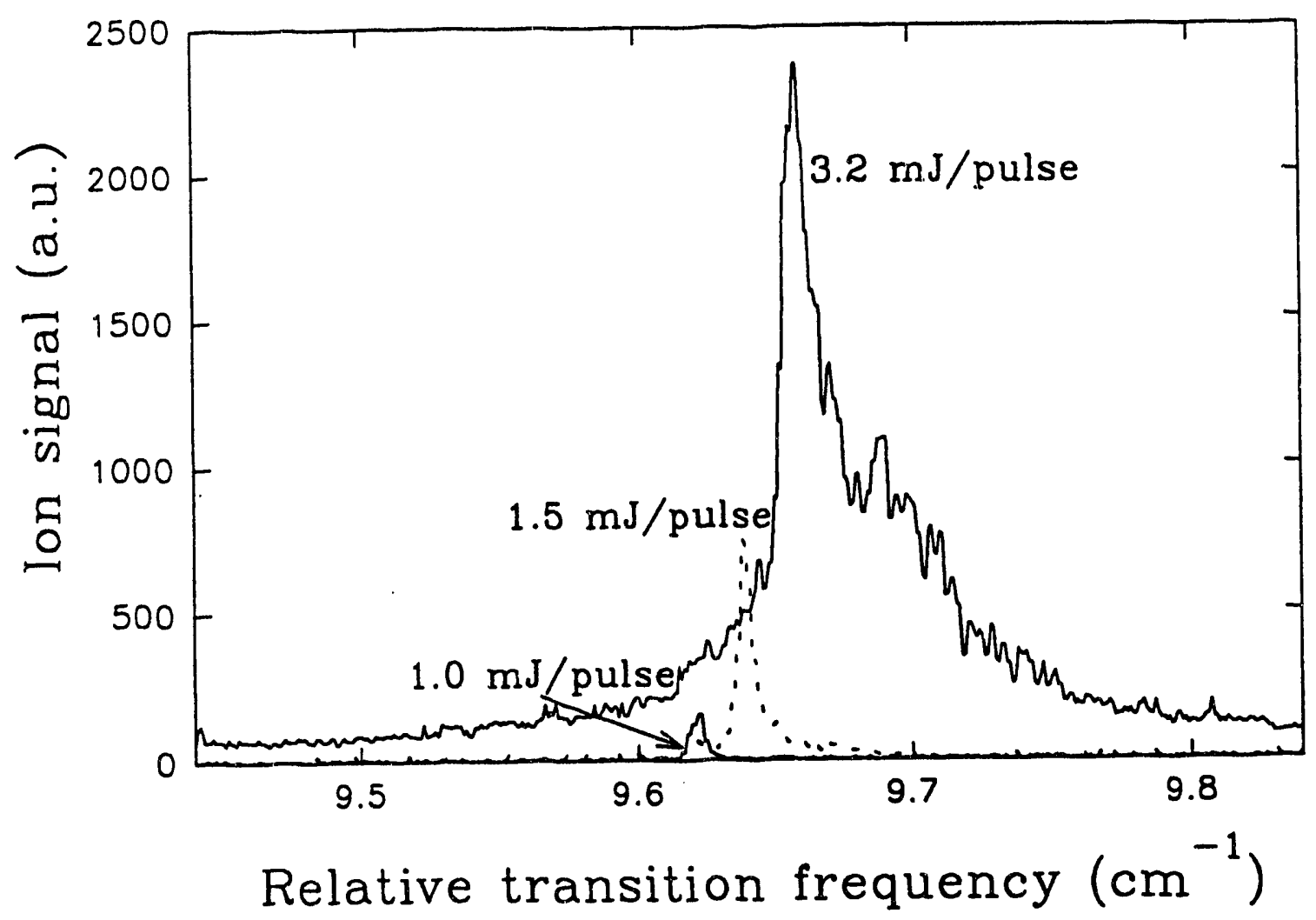

Figure 2 Frequency spectrum of $(2+1)$ REMPI of $\mathrm{H}_{2}(\mathrm{v}=0, \mathrm{~J}=1)$ at laser powers of $1.0 \mathrm{~mJ} / \mathrm{pulse}, 1.5 \mathrm{~mJ} / \mathrm{pulse}$ and $3.2 \mathrm{~mJ} / \mathrm{pulse}$. These laser powers were measured between the two $300 \mathrm{~mm}$ lenses of the telescope for the 201 $\mathrm{nm}$ light. The two-photon transition frequencies are obtained by adding $99100 \mathrm{~cm}^{-1}$ to the relative transition frequencies shown. 


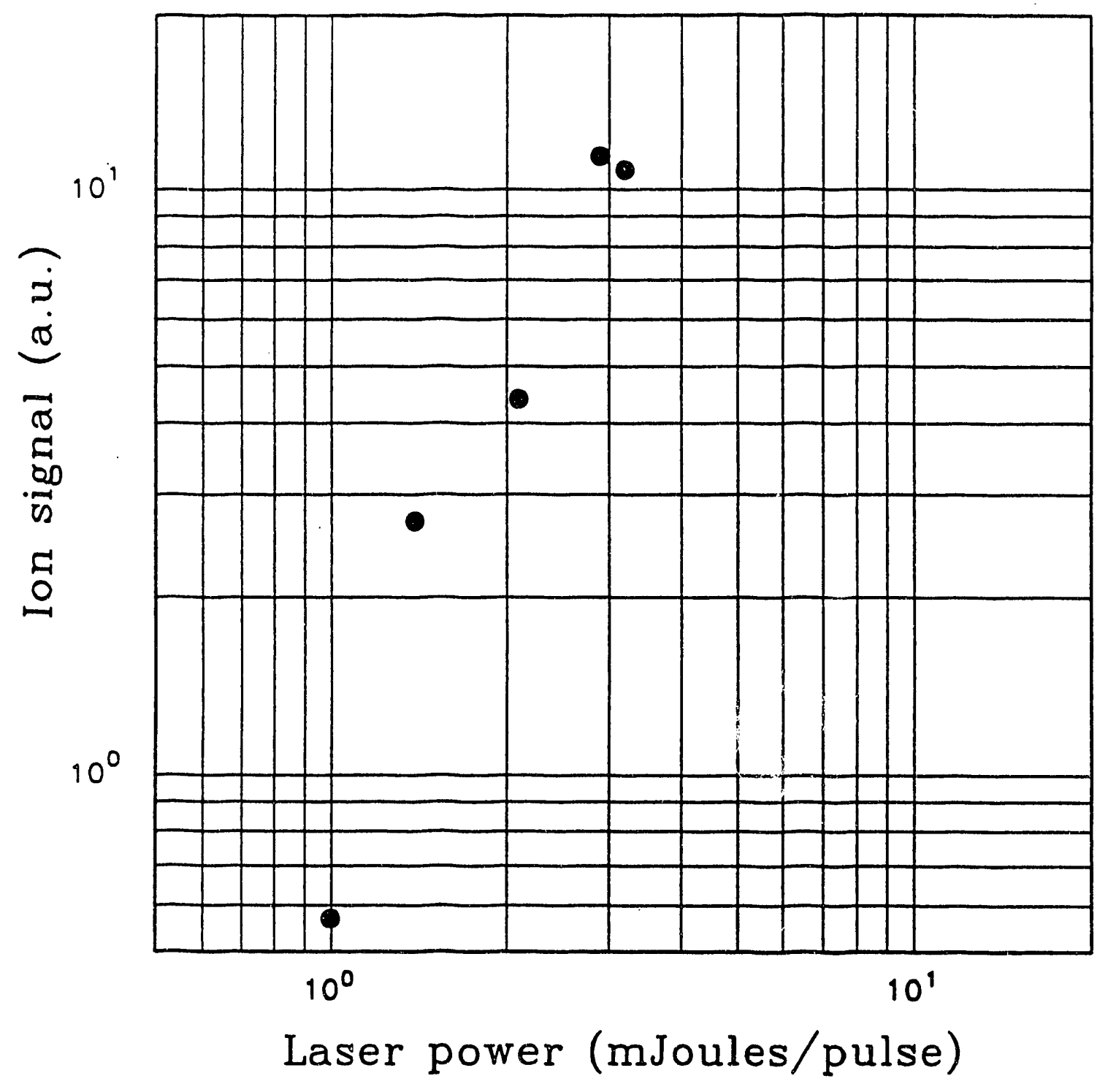

Figure 3 Double-log plot of the power dependence of $(2+1)$ REMPI of $\mathrm{H}_{2}(\mathrm{v}=0, \mathrm{~J}=1)$. The laser powers were measured between the two $300 \mathrm{~mm}$ lenses of the telescope for the $201 \mathrm{~nm}$ light. 
et.al. ${ }^{23)}$ have reported a shift of $45 \mathrm{~cm}^{-1}$ in the $\mathrm{E}, \mathrm{F}^{1} \Sigma_{\mathrm{g}}{ }^{+}(\mathrm{v}=2)<--\mathrm{X}^{1} \Sigma_{\mathrm{g}}{ }^{+}(\mathrm{v}=0)$ transition frequencies using ArF excimer laser powers of $610^{11} \mathrm{~W} / \mathrm{cm}^{2}$.

It is observed in Figure 2 that the AC Stark effect leads to a broadening of the linewidth beyond the laser linewidth, as well as an asymmetry of the lineshape. While the FWHM in the spectra recorded at $1.0 \mathrm{~mJ} /$ pulse and $1.5 \mathrm{~mJ} /$ pulse corresponds to the $150 \mathrm{MHz}$ two-photon laser bandwidth, the FWHM of the main peak in the spectrum recorded at a laser power of $3.2 \mathrm{~mJ} /$ pulse is equal to about $550 \mathrm{MHz}$. As a result of this broadening of the molecular linewidth, the effective line shape function $g_{\text {effective }}(\omega)$ in Equations (5) and (6) approaches $g_{M}(\omega)$, and the numerical pre-factor in Equation (9), which was calculated using a $150 \mathrm{MHz}$ two-photon bandwidth, will be reduced.

The ionization efficiency of $\mathrm{DH}(\mathrm{J}=0)$ by $(2+1)$ REMPI

A wavelength scan around the two-photon transition frequency for $\mathrm{DH} \mathrm{E}^{1} \Sigma_{g}^{+}(\mathrm{v}=0, \mathrm{~J}=0)$ $<-X^{1} \Sigma_{g}^{+}(v=0, J=0)$ is shown in Figure 4. This spectrum was recorded by admitting $\mathrm{H}_{2}$ gas into the vacuum chamber until the ionization gauge recorded a pressure increase of $510^{-7}$ Torr. Using the $\mathrm{H}_{2}$ molecule sensitivity factor of 2.4 recommended by Summers ${ }^{24)}$, the $\mathrm{H}_{2}$ pressure in the vacuum chamber was therefore equal to $1.210^{-6}$ Torr. The natural abundance of $D$ isotopes is $1.510^{-425}$. Consequently, under these experimental conditions the pressure of DH in the vacuum chamber was equal to 3.6 $10^{-10}$ Torr. At room temperature about $20 \%$ of the $\mathrm{DH}$ molecules are in the ground rotational state. Therefore the pressure of $\mathrm{DH}(\mathrm{v}=0, \mathrm{~J}=0)$ being probed was $7.210^{-11}$ Torr. The laser power inside the vacuum chamber in both counterpropagating laser beams was about $0.6 \mathrm{~mJ} /$ pulse.

The spectrum shown in Figure 4 was recorded by accumulating video images for about 20 lasershots per point while scanning the two-photon frequency at a rate of $30 \mathrm{MHz} / \mathrm{sec}$. At the maximum in Figure 1, the average number of $\mathrm{DH}^{+}$ions recorded per lasershot was equal to 190. As discussed in the experimental section, away from the two-photon resonance, the signal drops to less than one count per hundred 


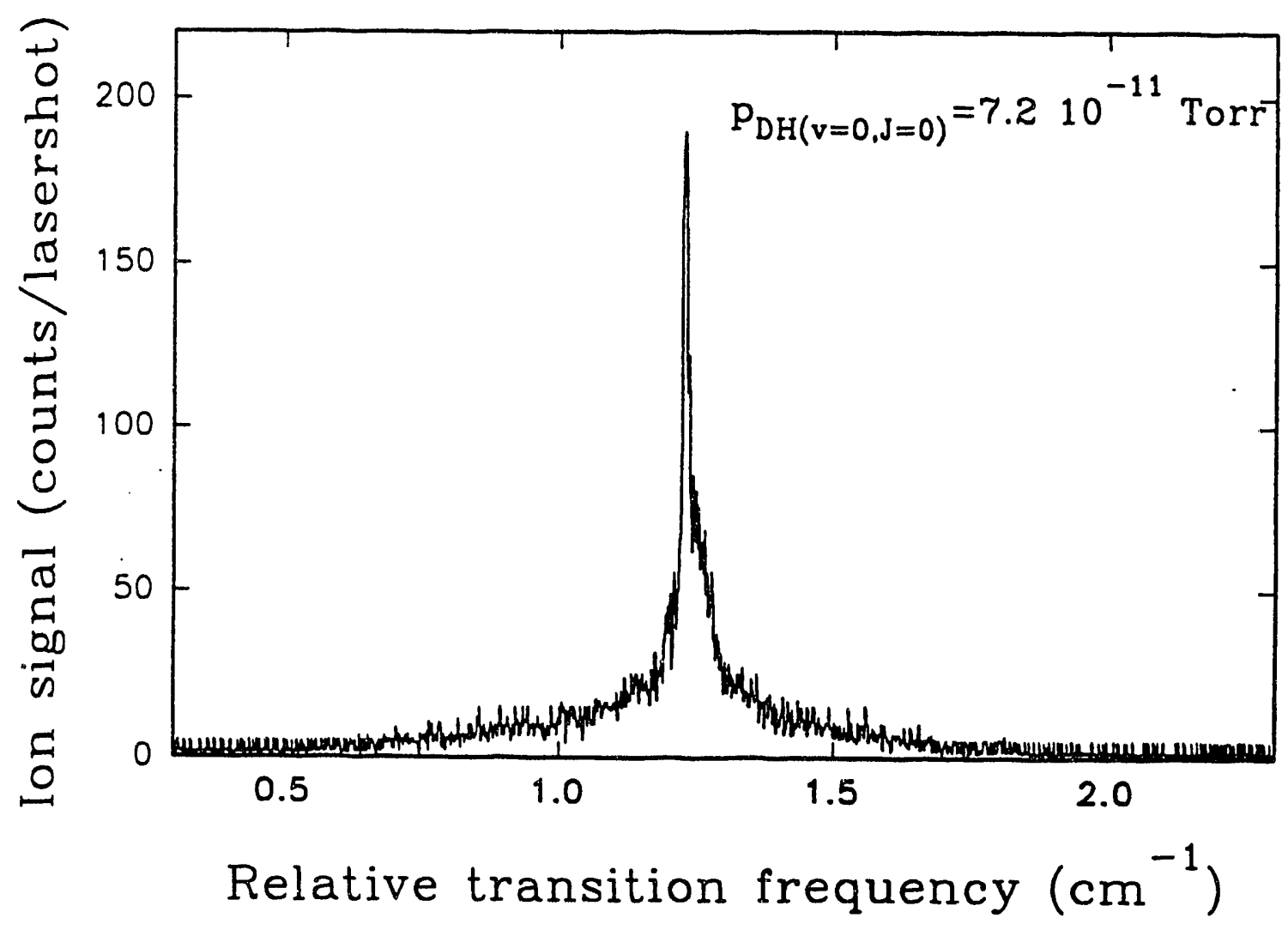

Figure 4 Frequency spectrum of (2+1) REMPI of $\mathrm{DH}(\mathrm{v}=0, \mathrm{~J}=0)$. The transition frequency is obtained by adding $99300 \mathrm{~cm}^{-1}$ to the relative transition frequency shown. 
lasershots. Therefore, a single shot signal-to-noise ratio of one would be obtained in a situation where on average one $\mathrm{DH}^{+}$molecule is detected per lasershot. This woulci be the case for a $\mathrm{DH}(\mathrm{v}=0, \mathrm{~J}=0)$ pressire of $3.810^{-13}$ Torr, or a density of $1.210^{4}$ $\mathrm{DH}(\mathrm{v}=0, \mathrm{~J}=0) / \mathrm{cm}^{3}$.

In the present experiment, the signal is limited by the quantum efficiency of the position-sensitive ion detector, which is about $60 \%$ for the microchannel plates used. If this detector were to be replaced by a more efficient detector such as a scintillation detector ${ }^{26)}$, the quantum efficiency could be improved to almost $100 \%$. In addition, if it is taken into account that the mesh which separates the acceleration region from the drifttube has a $90 \%$ transmission, the single shot detection limit for $\mathrm{DH}(\mathrm{v}=0, \mathrm{~J}=0)$ would be at a density of $6.810^{3} \mathrm{molecules} / \mathrm{cm}^{3}$.

As discussed in the experimental section, the spotsize inside the vacuum chamber was determined to be approximately $500 \mu \mathrm{m}$. Using Equation (9) the theoretical detection sensitivity for $\mathrm{DH}(\mathrm{v}=0, \mathrm{~J}=0)$ under the conditions described above was calculated to be approximately $2.010^{3}$ molecules $/ \mathrm{cm}^{3}$. There are two reasons why our experimental detection efficiency is smaller than this value. First, the spatial mode of the $201 \mathrm{~nm}$ light is not very homogeneous, and this may lead to an imperfect overlap of the counterpropagating beams througout the detection volume. More important however, as discussed above, the two-photon transitions are subject to a dynamic Stark effect which effectively broadens the molecular linewidth. Based on the observed FWHM of $550 \mathrm{MHz}$ in Figure 2, it is expected that the uppumping rate calculated using Equation (9) is too high by no less than a factor of four, and this readily accounts for the difference between the observed and the calculated detection efficiency. 


\section{Discussion}

In this chapter improved efficiencies have been reported for the detection of molecular hydrogen by the application of a $(2+1)$ REMPI scheme through the E,F ${ }^{1} \Sigma_{g}^{+}$state, using two counterpropagating near transform-limited laser beams. With readily available laser powers, a single shot $\mathrm{DH}(\mathrm{v}=0, \mathrm{~J}=0)$ detection efficiency of $6.810^{3}$ molecules $/ \mathrm{cm}^{3}$ has been demonstrated. Not only is this comparable or better than the best detection efficiency which has been obtained using $(1+1)$ xuv+uv REMPI, but due to the Doppler-free nature of the technique, this detection efficiency is independent of the velocity and angular distribution of the sample which is being probed.

In experiments involving state-specific spectroscopic product detection, a frequent problem is the fact that for many atoms and simple molecules the lowest optically accessible excited states are in the vacuum or extreme ultra-violet energy range. A choice has to be made between a multi-photon resonant excitation technique using ultraviolet lasers or a single-photon resonant excitation technique involving the use of coherent vacuum- or extreme ultraviolet laser light. While it is generally recognized that the generation of the light required in the multi-photon schemes is significantly easier, there is a consensus that the single-photon excitation schemes are more sensitive and that the resulting spectra are easier to analyze ${ }^{27.28)}$. We would like to present the argument that while single-photon excitation schemes are preferable in many applications, the individual details of an experiment determine which technique should be chosen.

The choice between a single- and a multi-photon excitation technique should be based on three criteria, namely, the sensitivity of the technique, the reliability of the obtainable experimental results, and the technical difficulty of the method. These criteria will now be discussed in turn.

In discussing the sensitivity of the single- and multi-photon excitation techniques, one of the most important factors is to consider the molecular and laser bandwidths involved in the experiment. The question that needs to be answered is to what extent the effective Dopplerwidth of the products to be detected limits the 
fraction of the products that can absorb within the bandwidth of the available laser system. If this fraction is significantly less than unity, the application of Doppler-free two-photon excitation may be appropriate.

For example, as demonstrated in this chapter, with a near-transform limited laser, single- and two-photon excitation of hydrogen molecules are competitive as long as the Doppler width is smaller than the laser bandwidth or other contributions to the molecular bandwidth. If the Doppler width becomes larger, as is frequently the case in dissocation processes or reactions involving hydrogen molecules, the sensitivity of the single-photon xuv excitation drops dramatically, while the sensitivity of the $(2+1)$ REMPI with counterpropagating beams is unaffected. Of course the sharp contrast that we observe under our experimental conditions between the detection efficiencies via $(1+1)$ REMPI and $(2+1)$ REMPI is a direct consequence of the use of a near transform-limited laser. In most laboratories, the bandwidth of the lasers used is significantly beyond the Fourier transform-limit, and the Doppler-free advantage may be lost. Also, since the mass of the hydrogen molecule is very light, the Doppler widths and the possible Doppler-free enhancements tend to be particularly large for hydrogen.

A second point which needs to be addressed is the reliability of the experimental results which can be obtained using single-photon and multi-photon resonant excitation schemes. A major advantage of the single-photon resonant techniques is the fact that extraction of the ro-vibrational population distributions is much easier using this method. Calculations of multi-photon transition moments are significantly more complicated than the evaluation of the Franck-Condon factors required when a single-photon resonant technique is used. For the case of the hydrogen molecule the situation is very fortuitous in that both for the one-photon excitation scheme $e^{29}$ and for the two-photon excitation scheme ${ }^{4,11)}$ extensive information on the line strengths is available.

It should be noted that in some experiments, a determination of the Doppler profile is one of the explicit goals. In experiments of this kind, a single-photon resonant or a single-beam multi-photon resonant technique will be preferable 
depending on whether the shot-to-shot fluctuations in the laser power are more pronounced in the vacuum ultraviolet generation or in the multi-photon excitation. In addition, in some experiments Doppler shifts can cause a non-uniform detection efficiency for different product angles and/or velocities. (2+1) REMPI with counterpropagating beams can be an appropriate way to circumvent these problems. Technically, the single-photon and multi-photon resonant schemes both have advantages and disadvantages. While application of the multi-photon techniques is possible using conventional tunable laser systems, the single-photon excitation sct $=$-mes require the sophistication of the generation of coherent vacuum or extreme ultraviolet light. The price that one pays in the multi-photon excitation schemes is that these experiments tend to be more sensitive to background signals. In the case of the hydrogen molecule, the REMPI lasers need to be focussed down to $O\left(10^{8} \mathrm{~W} / \mathrm{cm}^{2}\right)$. Not only does one need to worry about whether the REMPI laser will influence the molecular process under study, but at these high power densities, dissociation and/or ionization of background molecules can give additional signal. In our vacuum chamber, the background pressure is approximately $10^{-7}$ Torr, and without changing the apparatus, background-free detection would probably be limited to masses below $\mathrm{m}=25$. 


\section{REFERENCES}

1) For a review of these experiments see J.J. Valentini and D.L. Phillips, in "Advances in Gas-Phase Photochemistry and Kinetics - Bimolecular Collisions", edited by M.N.R. Ashfold and J.E. Baggott, (1989), p. 1-51.

2) M.J.J. Vrakking, A.S. Bracker and Y.T. Lee, Book of Abstracts, XIIIth International Symposium on Molecular Beams, El Escorial, Madrid, Span, 2-7 june 1991.

3) A.H. Kung, T. Trickl, N.A. Gershenfeld and Y.T. Lee, Chem. Phys. Lett. 144, 427 (1988).

4) K.-D. Rinnen, D.A.V. Kliner, R.N. Zare and W.M. Huo, Isr. J. of Chemistry 29. 369 (1989).

5) N. Bloembergen and M.D. Levenson, in "High-Resolution Laser Spectroscopy", edited by K. Shimoda, Topics in Applied Physics Vol. 13, (Springer, 1976), p. 315-369.

6) See, for example

a) C. Zimmermann, R. Kallenbach and T.W. Hänsch, Phys Rev. Lett. 65 , 571 (1990).

b) L. Julien, F. Biraben, J.C. Garreau and M. Alleguini, Laser Spectroscopy IX, Proceedings of the Ninth International Conference on Laser Spectroscopy, Bretton Woods, New Hampshire, June 18-23 1991, p. 341 .

c) S.A. Lee, S.J. Sternberg and L.U.A. Anderson, ibid; p. 382.

7) a) S. Chu, A.P. Mills, Jr. and J.L. Hall, Phys. Rev. Lett. 52, 1689 (1984).

b) M.S. Fee, A.P. Mills Jr., E.D. Shaw, R.J. Chichester, D.M. Zuckerman, S. Chu and K. Danzmann, Phys. Rev. A 44 , R5 (1991).

8) D.J. Kligler and C.K. Rhodes, Phys. Rev. Lett. 40, 309 (1978).

9) E.E. Marinero, R. Vasudev and R.N. Zare, J. Chem. Phys. 78, 692 (1983).

10) E.E. Eyler, J. Gilligan, E. McCormack, A. Nussenzweig and E. Pollack, Phys. Rev. A 36, 3486 (1987). 
11) a) W.M. Huo, K.-D. Rinnen and R.N. Zare, J. Chem. Phys. 95, 205 (1991).

b) K.-D. Rinnen, M.A. Buntine, D.A.V. Kliner, R.N. Zare and W.M. Huo, J. Chem. Phys. 95, 214 (1991).

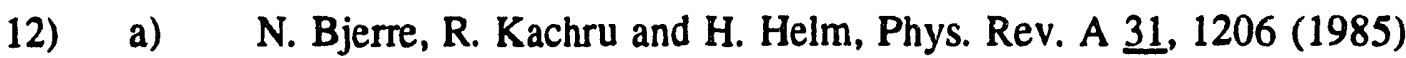

b) E.Y. Xu, H. Helm and R. Kachru, Phys. Rev. A 프, 3979 (1989).

13) a) W.L. Glab and J.P. Hessler, Phys. Rev. A. $\underline{35}, 2102$ (1987); ibid $\underline{42}$, 5486 (1990).

b) W.L. Glab and J.P. Hessler, Phys. Rev. Lett. 62, 1472 (1989).

14) For a review of the perturbation theory for one- and two-photon transitions see W.M. McClain and R.A. Harris in "Excited States", Vol. 3, edited by E.C. Lim, (Academic, 1977), p. 1-56.

15) D. Kligler, J. Bokor and C.K. Rhodes, Phys. Rev. A 21, 607 (1980).

16) a) E.F. Cromwell, PhD thesis, University of Califomia at Berkeley (1991).

b) E.F. Cromwell, private communication.

17) E. Cromwell, T. Trickl, Y.T. Lee and A.H. Kung, Rev. Sci. Instrum. 60, 2888 (1989).

18) J.M. Khosrofian and B.A. Garetz, Appl. Opt. 22, 3406 (1983).

19) a) K.R. Lykke and B.D. Kay, J. Chem. Phys. 95, 2252 (1991).

b) M.J.J. Vrakking, A.S. Bracker and Y.T. Lee, J. Chem. Phys. 96, 7195 (1992).

20) R.A. Wick, BioTechniques I, 262 (1989).

21) P.F. Liao and J.E. Bjorkholm, Phys. Rev. Lett. $\underline{34}, 1$ (1975).

22) J.P. Hessler, private communication.

23) a) T. Srinivasan, H. Egger, H. Pummer and C.K. Rhodes, I.E.E.E. Journal of Quantum Electronics, $\mathrm{OE}-19,1270$ (1983).

b) H. Pummer, H. Egger, T.S. Luk, T. Srinivasan and C.K. Rhodes, Phys. Rev. A 28, 795 (1983).

24) R.L. Summers, NASA Technical Note TN D-5285.

25) CRC Handbook of Chemistry and Physics. 
26) N.R. Daly, Rev. Sci. Instrum. 31, 264 (1960).

27) J.W. Hepburn, in "Vacuum Ultraviolet Photoionization and Photodissociation of Molecules and Clusters", edited by C.Y. Ng (World Scientific, 1991), p. 435485.

28) A.H. Kung and Y.T. Lee, ibid., p. 487-502.

29) H. Abgrall, F. Launay, E. Roueff and J.-Y. Roncin, J. Chem. Phys. $\underline{\text { 87, } 2036}$ (1987). 


\section{CHAPTER III}

\section{DESIGN OF A NEW CROSSED MOLECULAR BEAM EXPERIMENT}

TO MEASURE FULLY STATE-RESOLVED ANGULAR DISTRIBUTIONS FOR THE HYDROGEN EXCHANGE REACTION 


\begin{abstract}
ABSTRAC' $\Gamma$
In this chapter a description will be given of the design of a new experiment aimed at measuring fully state-resolved differential cross sections for the hydrogen exhange reaction $\mathrm{D}+\mathrm{H}_{2}-\rightarrow \mathrm{DH}(\mathrm{v}, \mathrm{j})+\mathrm{H}$. This experiment is a crossed molecular beam experiment, in which DH molecules formed in the reaction are ionized using the Doppler-free $(2+1)$ Resonance-Enhanced Multi-Photon Ionization (REMPI) technique described in Chapter II, and detected using a position-sensitive ion detector. Following a brief introduction outlining the scientific interest in studying this reaction, a detailed description of the experimental approach and the feasibility of the experiment are presented.
\end{abstract}




\section{Introduction}

Over the past decade the progress that has been made by both theoreticians and experimentalists in studying the hydrogen exchange reaction $\mathrm{H}+\mathrm{H}_{2}(\mathrm{v}, \mathrm{J}) \rightarrow->\mathrm{H}_{2}\left(\mathrm{v}^{\prime}, \mathrm{J}^{\prime}\right)$ $+\mathrm{H}$ (and its isotopic variants) has been nothing short of sensational. On the theoretical front new techniques developed simultaneously by Zhang and Miller, and by Truhlar and Kouri and co-workers ${ }^{1)}$, have enabled the first fully converged quantum mechanical scattering calculations for a number of simple systems. What makes the hydrogen exchange reaction particularly noteworthy in this regard is the fact that it remains the only reaction to date for which there a potential energy surface available ${ }^{2}$, which is believed to be 'chemically accurate'. Therefore it is of great interest to study this reaction as detailed as possible at the experimental level.

The most detailed conceivable experimental study of the hydrogen exchange reaction would consist of a measurement of the state-to-state differential cross section (preferably as a function of collision energy). This kind of experiment has not been possible to date, though recent progress has been impressive ${ }^{3)}$. Much of this progress has been due to the recent availability of high power laser systems. Whereas earlier studies of reactions involving unstable species like $\mathrm{H}$ atoms had to rely on techniques like pyrolysis and flash photolysis, it has become possible, using high power excimer or Nd:YAG lasers, to generate $D / H$ atoms by photolysis of a suitable precursor molecule in sufficient amounts to carry out a host of reactive studies. Most remarkable in this sense was certainly the measurement of the differential cross section for the $D$ $+\mathrm{H}_{2}-->\mathrm{DH}+\mathrm{H}$ reaction by Continetti et. al."). In this experiment a pulsed beam of D atoms, generated by photolysis of deuterium iodide (DI), was crossed with a pulsed beam of $\mathrm{H}_{2}$, and $\mathrm{DH}$ molecules formed in the reaction were measured using a time-offlight electron bombardment mass spectrometer, allowing for a measurement of the differential cross section with vibrational state resolution. The resolution in this experiment was limited by the pay-off that exists in this kind of experiment between the minimum detection efficiency required to obtain a detectable signal and the finite size of the electron bombardment ionizer required to achieve this. 
Lasers have also figured extensively in the detection of molecules formed in the hydrogen exchange reaction. Using the techniques ${ }^{5)}$ of coherent anti-Stokes Raman scattering (CARS) and the more sensitive technique of resonance-enhanced multiphoton ionization (REMPI) Valentini and co-workers, and Zare and co-workers were able to determine final ro-vibrational product state distributions for the various isotopic variants of the hydrogen exchange reaction at a large number of collision energies ${ }^{3}$. The next level of detail could be achieved by a synthesis of the experimental techniques employed by Continetti et. al. and the schemes used by Zare and Valentini, i.e. a measurement of the fully-state resolved differential cross sections in a crossed molecular beam experiment with laser-based detection of the reaction products. With a laser detection scheme resolution of the final ro-vibrational state is inherent and, additionally, as will be demonstrated below, by applying the Doppler-free $(2+1)$ REMPI scheme discussed in the previous chapter, it will prove to be possible to increase the detection efficiency of the reaction products, which is rather low when employing an electron bombardment technique. For example, in the experiment of Continetti et. al., only about $0.2 \mathrm{DH}$ molecules were detected per reaction cycle ${ }^{6}$ since the ionization efficiency of the ionizer was about $610^{-6}$.

In the remainder of this chapter the design of an experiment will be discussed which aims at measuring the fully state-resolved differential cross sections for the hydrogen exchange reaction. A scheme will be described where the angular distribution for a selected ro-vibrational state will be determined in a crossed molecular beam experiment employing a combination of $(2+1)$ REMPI and positionsensitive ion detection. First however, a few more introductory remarks will be made.

Observations by Valentini et. al.") of structure in the collision energy dependence of the total cross section for formation of specific final ro-vibrational product states sparked an intense debate as to whether Feshbach-type dynamical resonances play a role in the hydrogen exchange reaction. The rationale for the occurrence of these resonances is that, whereas the reaction is predominantly vibrationally adiabatic, there does exist some coupling between the vibrationally adiabatic ground state surface, correlating to $\mathrm{H}+\mathrm{H}_{2}(\mathrm{v}=0)$, and the vibrationally 
adiabatic surface correlating to $\mathrm{H}+\mathrm{H}_{2}(\mathrm{v}=1)$. If the total energy of the triatomic system coincides with a quasi-bound vibrational state on the latter surface, the reactive cross sections can be strongly affected ${ }^{8}$. Valentini's results strongly contradicted the converged quantum calculations by Zhang and Miller ${ }^{9}$, which did not show any marked structure in the energy dependence of the integral cross section. After their publication the validity of Valentini's experimental results was convincingly refuted by Zare et. al. ${ }^{10)}$, but the question as to whether dynamical resonances do play a role remains a very interesting one. In fact, a more detailed inspection of the theoretical results of Zhang and Miller brought out previously overlooked features in the state-tostate differential cross sections which could be attributed to dynamical resonances ${ }^{11)}$. It was clear from these theoretical results that, rather than in the integral cross section, the only way in which the influence of dynamical resonances can be demonstrated experimentally is through a measurement of the state-resolved differential cross section. The state-resolved differential cross sections can be thought of as the sum of a set of angular distributions corresponding to different values of the total angular momentum of the triatomic system. In the absence of molecular rotation, this angular momentum is closely related to the impact parameter for the collision. As the collision energy is tuned in the energy range where the resonance occurs, different angular momentum states come into resonance when the sum of the (vibrational) resonance energy and the rotational energy of the complex matches the total energy. At this point the angular distribution can show a marked change, reflecting a change in the contribution of the resonant angular momentum state(s). Specifically, Miller has shown that in addition to the dominant backscattered molecular product (with respect to the initial $\mathrm{D} / \mathrm{H}$ atom direction in the center-of-mass frame) resulting from the direct reaction, the occurrence of a dynamical resonance leads to the formation of a small second peak in the angular distribution which moves to forward angles as the energy (and the angular momentum state which causes the resonance) is tuned. On. if the main goals of the experiment described in this chapter is to explore if, as predicted by the theory, at specific collision energies the angular distributions will show the influence of dynamical resonances. 
Another potentially very interesting aspect of the experiments discussed in this chapter evolves around the observability of the geometric phase (sometimes referred to as Berry's phase) in reactive systems ${ }^{12}$. As discussed by Herzberg and LonguetHiggins, the electronic wavefunction changes sign over a path in nuclear configuration space that goes around a conical intersection ${ }^{13)}$. This means that when the BornOppenheimer separation of nuclear and electronic variables is carried out and the equation for the nuclear wavefunction is solved, the nuclear wavefunction is not a continuous and single valued function, unless a vector potential ten is added to the Schrodinger equation ${ }^{14)}$. This is of interest in the case of $D / H+H_{2}$, because the ground and first electronically excited state are part of a ${ }^{2} E$ ' state whose degeneracy is removed by the Jahn-Teller effect, resulting in a conical intersection in equilateral triangular geometries. Kupperman and co-workers have performed converged quantum calculations exploring the consequences of inclusion of the geometric phase ${ }^{15)}$. They have shown $\mathrm{t}$ at for $\mathrm{H}+$ para- $\mathrm{H}_{2}-\rightarrow \mathrm{H}+$ para- $\mathrm{H}_{2}$ and $\mathrm{H}+$ ortho- $\mathrm{H}_{2}-\rightarrow \mathrm{H}+$ ortho- $\mathrm{H}_{2}$, where the thre $\mathrm{e}$ atorns are indistinguishable, the interference in the differential cross section between direct (non-reactive) and exchange (reactive) collisions is inverted when the geometric phase is included. For the case of $D+\mathrm{H}_{2}$, one might anticipate an interference at relatively high collision energies of perhaps $2 \mathrm{eV}$, when reactive trajectories encircling the conical intersection become feasible. A new and very exciting result has been reported in studies of $D+\mathrm{H}_{2}(v=1)$, where discrepancies between experimental and theoretical product state distributions have been removed by inclusion of the geometric phase ${ }^{16)}$.

Before proceeding to a detailed description of the proposed technique, attention needs to be called to a complementary experiment which is currently in progress. In Welge's group in Germany, work is currently under way to measure fully stateresolved differential cruss sections for the $\mathrm{H}+\mathrm{D}_{2}--\rightarrow \mathrm{DH}+\mathrm{D}$ reaction. ${ }^{17}$ ) Similar to Continetti et. al. ${ }^{4}$ ) this study is a crossed molecular beam experiment with a time-offlight technique for the energy analysis of the products. Instead of electron bombardment ionization of the molecular fragment however, the $D$ atom that results from the reaction is excited to a high-n Rydberg state using two-color vacuum- 
ultraviolet and ultraviolet excitation, and subsequently field-ionized after having travelled to the detector. Following from the geometry of the detection scheme, the intrinsic energy resolution that can be obtained in this scheme is much better than a conventional time-of-flight detector with electron bombardment $(\Delta E / E \approx 0.003$ at $E=1$ $\mathrm{eV}$ ), and Welge et.al. have been able to resolve structure in the $\mathrm{D}$ atom time-of-flight that reflects the rotational and vibrational energy of the DH molecule after the reaction.

2. Description and feasibility analysis of the proposed technique to measure fully state-resolved angular distributions

In this section the basic principle of the new experiment to measure fully-state resolved angular distributions will be discussed and a calculation will be presented which was done to assess the feasibility of the experiment. In as far as the crossed molecular beam set-up is concerned, the experimental approach leans heavily on the experience gained by Continetti et.al. ${ }^{4.6}$, indeed the crossed molecular beam set-up is little more than a scaled down version of the former experiment. The new ingredient in the current experiment is the detection scheme discussed in chapter II. After discussing the general principle of the experiment in section 2.1, the achievable production of $\mathrm{DH}$ from the reaction of $\mathrm{D}+\mathrm{H}_{2}$ in will be calculated in section 2.2. Section 2.3 discusses how the experiment becomes feasible when Doppler-free $(2+1)$ REMPI using counterpropagating ultraviolet lasers is employed.

\subsection{The basic principle and the experimental set-up}

In Figure 1 a schematic representation of the experiment is shown. The set-up is basically a crossed molecular beam arrangement in which pulsed beams of $D$ atoms and $\mathrm{H}_{2}$ molecules cross at right angles and $\mathrm{DH}$ molecules formed in the reaction are ionized by a laser at some distance from the crossing point. The ions are mapped onto a position-sensitive detector. Before going into the details of the crossed molecular 


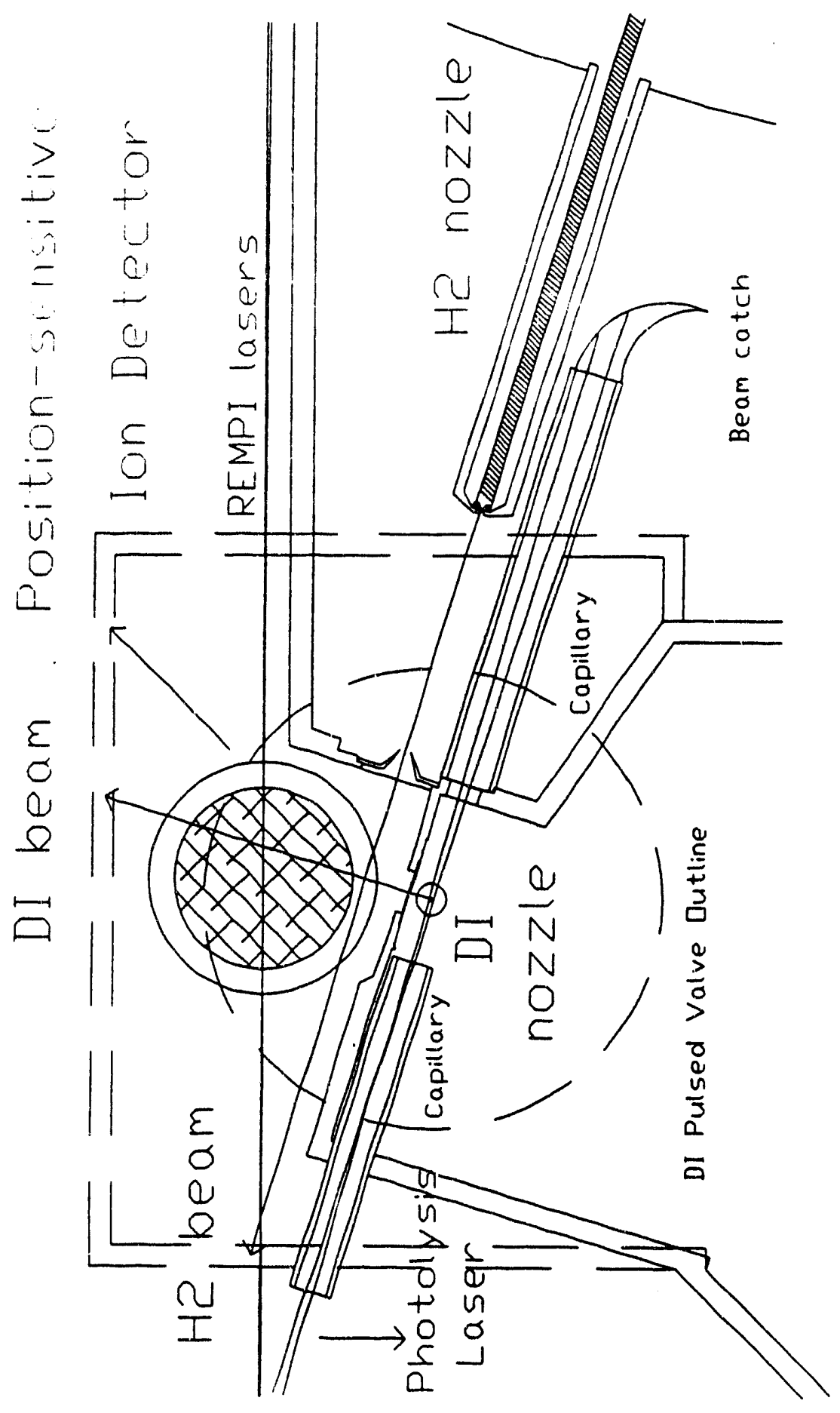

Figure 1 Schematic representation of the experiment designed to measure rovibrationally state-resolved differential cross sections for the $\mathrm{D}+\mathrm{H}_{2}-\rightarrow$ $\mathrm{DH}(v, \mathrm{j})+\mathrm{H}$ reaction. See text for details. 
beam arrangement, we will discuss how a set-up like the one sketched above allows for a measurement of the state-resolved angular distributions.

The principle of the angular distribution measurement for a particular rovibrational product state is explained in Figure 2 . In velocity space the $D$ atom velocity and the $\mathrm{H}_{2}$ velocity combine to give a center-of-mass velocity and a relative velocity, with the latter determining the collision energy. After reaction, for a given ro-vibrational product state $\mathrm{DH}(\mathrm{v}, \mathrm{J})$, the internal energy is a constant and thus the energy partitioned into the relative motion of the $\mathrm{DH}$ and $\mathrm{H}$ is fixed. Therefore in the center-of-mass frame the magnitude of the $\operatorname{DH}(\mathrm{v}, \mathrm{J})$ velocity is fixed and in the laboratory the DH products are found on an expanding globe moving around the center-of-mass direction, with cylindrical symmetry of the product distribution around the direction of the relative velocity vector of the $D$ and $\mathrm{H}_{2}$ beams. A complete angular distribution can be obtained from an experiment where the distribution of the products is measured in a plane including the relative velocity vector of the two beams. In most conventional crossed molecular beam experiments this is achieved by recording time-of-flight mass spectra in the plane of the two beams at different angles with respect to the center-of-mass direction. This means that in velocity space the measurement consists of successive intersections of the product circle with lines passing through the origin. In the current scheme a laser axis is defined at some distance from the crossing point of the beams and the measurement of the angular distribution is carried out as follows. A choice of the time-delay between the photolysis laser and the detection laser selects a value $v_{x}$ for the $\mathrm{DH}$ velocity perpendicular to the laser axis, since, due to the monochromaticity of the $D$ atom velocity, the moment at which reactions take place is well-defined. Measurement of the position of detected product molecules along the laser axis, using a positionsensitive ion detector, allows for a determination of the second planar velocity component $v_{y}$, and thus, by varying the time-delay between the two lasers, a complete mapping of the product distribution $I\left(v_{x}, v_{y}\right)$ is possible, giving a very direct measurement of the angular distribution. Comparing this method with the way the angular distribution is measured in a conventional experiment, we see that.the current 
Figure 2 Principle of the state-resolved angular distribution measurements for the $D+\mathrm{H}_{2} \rightarrow \mathrm{DH}(\mathrm{v}, \mathrm{j})$ reaction proposed in this chapter. The Newton diagram under typical experimental conditions is shown on the left. It illustrates how the D- and $\mathrm{H}_{2}$ velocities combine to give a center-ofmass velocity, and how, in the experimental plane, the $\mathrm{DH}$ product molecules in a selected final ro-vibrational product state lie on a circle around the center-of-mass velocity vector. The figure on the right shows how the distribution of DH product molecules on this circle is determined by measuring the ion distribution along the laser detection axis as a function of the time delay between the firing of the detection laser and the time of reaction. 


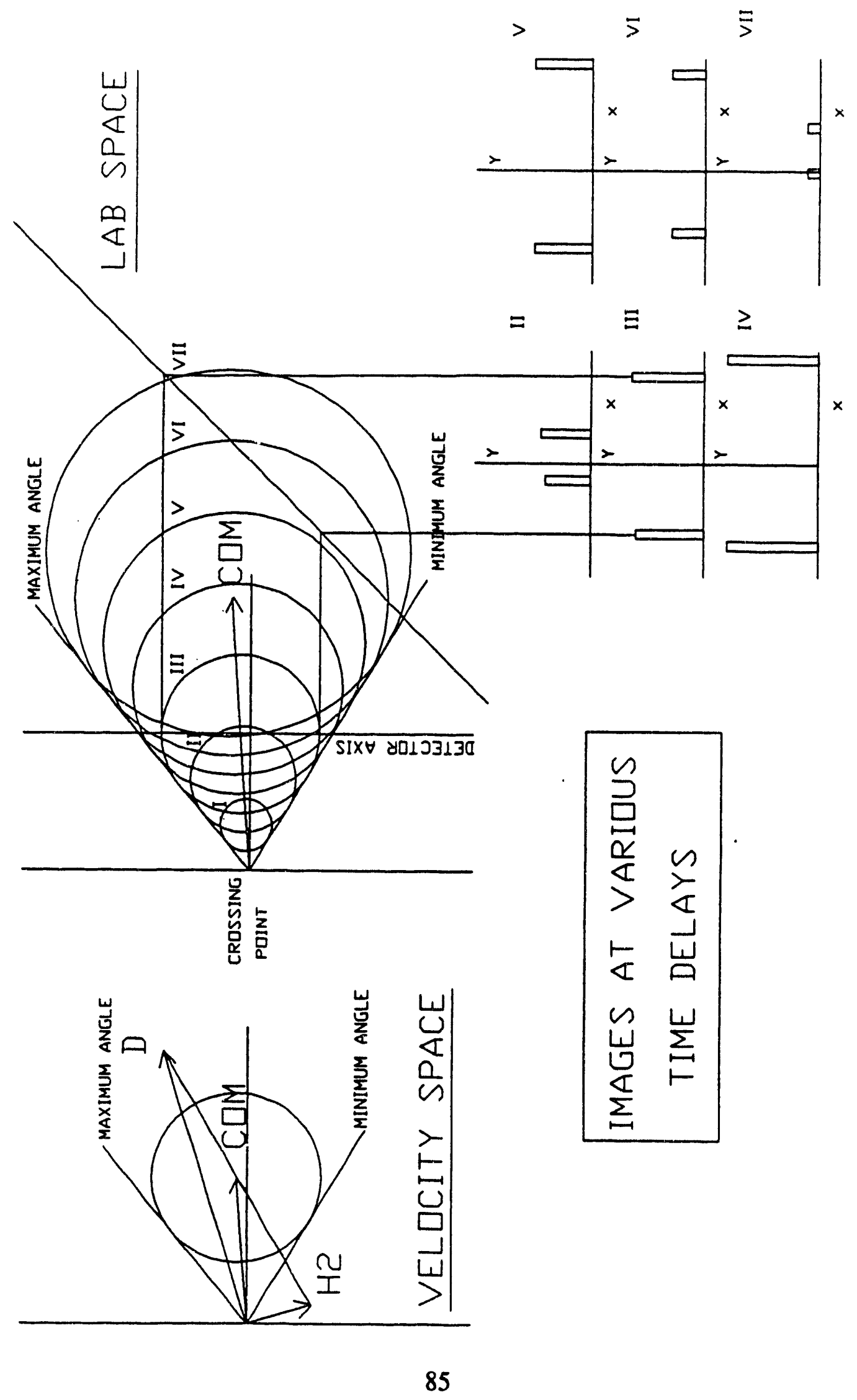


measurement corresponds to making successive intersections with the product circle along lines that run parallel with eachother. It should be pointed out that in the analysis of an actual experiment the velocity components $v_{x}$ and $v_{y}$ need not necessarily be explicitly evaluated, but a combination of linear and non-linear least squares routines are used to determine the angular distributions and the parameters describing the experimental conditions, which best reproduce the measured set of onedimensional ion distributions along the laser axis.

In order to carry out an experiment using the scheme described above, a vacuumchamber was constructed, consisting of three separate chambers, namely, i) the DI chamber, in which the D atoms are generated, ii) the $\mathrm{H}_{2}$ chamber, in which the $\mathrm{H}_{2}$ beam is generated, and iii) the main chamber, in which the $\mathrm{D}$ and $\mathrm{H}_{2}$ beams cross, and the reaction products are ionized and subsequently detected on the position-sensitive ion detector.

In the DI chamber deuterium atoms are generated at the intersection of a pulsed beam of DI (perpendicular to the plane of Figure 1) and the photolysis laser. The pulsed DI beam is generated using a home-built piezoelectric pulsed valve, which is a modified version of the design of Proch and Trickl ${ }^{18}$. This valve works on the principle that a $0.5 \mathrm{rm}$ diameter nozzle is opened for approximately $250 \mu \mathrm{sec}$ when an $\mathbf{O}$-ring seal, mounted on a plunger attached to a piezoelectric crystal (Model $\mathbf{P}$ 910.160 disk translator, Physik Instrumente $\mathrm{GmbH}$ ), is pulled back. Because of geometrical constraints in the experiment, the nozzleplate in Proch and Trickl's design is replaced by a $5 \mathrm{~cm}$ long tube with a nozzle opening at the end. The pulsed valve is typically mounted with the nozzle about $0.7-1.2 \mathrm{~mm}$ below the reaction plane. Because of the corrosive properties of DI, it is necessary to protect the piezoelectric crystal, which is achieved by the application of Fomblin perfluorinated grease. Like all other pulsed components of the experiment, the $\mathrm{D}$ atom generation is performed at a repetition rate of $10 \mathrm{~Hz}$.

The DI chamber is pumped by a Varian HS-6 diffusion pump. In addition, to avoid corrosion of the mechanical pump backing the diffusion pump, the $\mathrm{DI}$ is cryopumped on a series of $\mathrm{LN}_{2}$-cooled cryopanels facing the beam. From a 
comparison between operation of the valve with DI and operation with $\mathrm{N}_{2}$, it is estimated that in excess of $98 \%$ of the DI is frozen in on the cryopanels. The DI is made following the method described in reference 6 .

An ultra-violet photolysis laser is required to dissociate the DI molecule $\left(D_{0}=\right.$ $3.094 \mathrm{eV}^{19)}$ ) and to give the $\mathrm{D}$ atom sufficient energy to allow the $\mathrm{D}+\mathrm{H}_{2}$ reaction, which has a threshold of about $0.3 \mathrm{eV}$, to occur. In the photodissociation of DI the iodine atom is formed in two spin-orbit states

$$
\begin{aligned}
& D I \stackrel{h v}{\longrightarrow} D+I\left({ }^{2} P_{3 / 2}\right) \quad \text { \& transition } \\
& D I \stackrel{h v}{\longrightarrow} D+I \cdot\left(P_{1 / 2}\right) \quad \text { transition }
\end{aligned}
$$

which are separated by $0.943 \mathrm{eV}^{20)}$. In the initial experiments the 4th harmonic of a Nd:YAG laser (266 nm) is used to photodissociate the DI, giving fast D atoms with a kinetic energy of $1.54 \mathrm{eV}$, and slow D atoms with a kinetic energy of $0.61 \mathrm{eV}$. By choosing the polarization of the photolysis laser perpendicular to the plane of reaction the fast $D$ atoms are preferentially moving in the plane of reaction (perpendicular to the laser polarization direction), whereas the slow D atoms are preferentially directed out of the plane of reaction along the laser polarization direction. As can be seen in the Newton diagram in Figure 2, if a photolytic D atom source is used the collision energy for the $\mathrm{D}+\mathrm{H}_{2}$ reaction is mostly determined by the $\mathrm{D}$ atom velocity. Therefore, the collision energy dependence of the differential cross sections, and the possible occurrence of dynamical resonances, can conveniently be studied by using a readily available tunable photolysis laser.

Existing geometrical constraints demand that the photolysis laser has to pass through the main chamber before accessing the DI beam. Since there are no window materials that can withstand the laser power levels required in the photolysis 
process, the photolysis laser enters the DI chamber through a $5 \mathrm{~cm}$ long $0.5 \mathrm{~cm}$ ID tube. The conductance of this tube is only $0.25 \mathrm{l} / \mathrm{s}$ and therefore this tube has only a negligible influence on the pressure differential between the main chamber and the DI chamber. A similar tube, ending in a woodshorn, separates the DI chamber from the $\mathrm{H}_{2}$ chamber. The photolysis laser is focussed using a $50 \mathrm{~cm}$ fused silica lens with the focus a few centimeters before the DI beam. The location of the focus is manipulated so that the size of the laser beam matches the size of the expanding DI jet. Careful adjustment is important for two reasons. If the laser beam is larger than the DI pulsed jet, valuable photons are wasted and the fraction of $D$ atoms dissociated and the intensity of the $D$ atom beam are smaller than they could be. On the other hand, if the laser beam is smaller than the DI pulsed jet, the D atoms will encounter an area of undissociated DI on their way to the main chamber, which will hurt the collimation and the monochromaticity of the $\mathrm{D}$ atom beam.

The $\mathrm{D}$ atoms enter the main chamber through a variable size aperture located $3.6 \mathrm{~mm}$ away from the center of the DI nozzle and cross the $\mathrm{H}_{2}$ beam at a distance of $10.1 \mathrm{~mm}$ from this point. The pulsed valve used for generating the $\mathrm{H}_{2}$ beam is very similar to the one used for the DI beam. The $\mathrm{H}_{2}$ jet is skimmed by an electro-formed nickel skimmer (Beam Dynamics). Some experiments were also done with a quasidoubly differential pumping arrangement, where the $\mathrm{H}_{2}$ jet was skimmed first by an electro-formed nickel skimmer, mounted on a small cone, which was displaced by about $1 \mathrm{~cm}$ from the wall which separates the $\mathrm{H}_{2}$ chamber from the main chamber. A home-machined aluminum skimmer was mounted on this second wall. The rationale for this arrangement was that in initial experiments, with a single skimmer, some nonresonant noise signal could be detected when the detection laser and the $\mathrm{H}_{2}$ beam were operated. This was thought to be due to effusion of $\mathrm{H}_{2}$ molecules from the $\mathrm{H}_{2}$ chamber, which greatly increased the $\mathrm{H}_{2}$ pressure in the detector volume. Introducing a second differential wall reduces the pressure build-up during the pulse in front of the final skimmer, since the molecules which do not pass through the first skimmer are effectively blocked from reaching the second skimmer on the timescale of the experiment. As discussed in the next chapter however, it was not possible to obtain 
sufficiently intense $\mathrm{H}_{2}$ beams with this arrangement. The $\mathrm{H}_{2}$ chamber is pumped by a water-cooled Varian HS-10 diffusion pump.

In the design of the experiment as it is shown in Figure 1, the perpendicular displacement of the detection laser axis from the crossing point is $15 \mathrm{~mm}$. There are several reasons for the choice of this dimension. On one hand one needs sufficient distance between the crossing point and the detector axis, to allow for the product circle to expand to the point where the different $\left(v_{x}, v_{y}\right)$ components can be wellresolved spatially. At a distance of $15 \mathrm{~mm}$, for a typical ro-vibrational product state, the average point on the product circle is probed when the diameter of the circle is about $1 \mathrm{~cm}$. As will be shown later in the signal calculation, a reasonable first order experiment is possible under this condition with what will be considered a minimum acceptable resolution, where all resolution defining elements (nozzles, skimmers) are on the order of $1 \mathrm{~mm}$. In order to allow for improvements in the resolution of the experiment once the basic geometry has been established, it is necessary that the minimum acceptable resolution can be achieved under conditions where in subsequent experiments it is still relatively easy to decrease the size of the resolution defining elements.

There is also an upper limit on the distance between the crossing point and the detector axis. First of all, the density of the number of product molecules in the viewline of the laser decreases with the square of the radius of the product circle. In addition, probing of larger product circles requires larger position-sensitive detectors and gating of the microchannel plates used in the detectors, which is crucial to inhibit detection of ions at masses other than the desired one, becomes increasingly hard, since the capacitance of the microchannel plates used in the detector increases with the square of the diameter of the plates. The current design employs a position-sensitive detector with a diameter of $32 \mathrm{~mm}$, where gating with a rise- and fall times of about 5 nsec is still achievable.

As shown in Figure 1, the $D$ and $\mathrm{H}_{2}$ beam are oriented at an angle of $17^{\circ}$ with respect to the detection laser axis. There are several reasons for choosing this orientation. First of all, this orientation serves to rotate the main body of the $\mathrm{H}_{2}$ pulsed 
valve out of the way of the detector laser axis. However, there is a another, considerably more important reason for this choice, which may not be immediately apparent. The choice of a $17^{\circ}$ angle orients the center-of-mass velocity axis for the $D$ $+\mathrm{H}_{2}$ reaction close to perpendicular with the detection laser axis for typical D atom kinetic energies, and this means that the range of angles of the $\mathrm{DH}$ product trajectories with respect to the detection laser axis is minimized. In other words, in this orientation the range of the laser axis where $\mathrm{DH}$ molecules cross the axis is minimized. As a consequence the crossing point of the beams can be put relatively farther away from the center of the position-sensitive detector and, therefore, closer to the DI nozzle. If the center-of-mass velocity axis were to be rotated significantly with respect to its current near-perpendicular geometry, it would be necessary to locate the crossing point inside the position-sensitive detector in order to keep all $\mathrm{DH}$ product trajectories inside the range of the detector. As a consequence the $D$ atom flux at the interaction region would be lower and, also, concerning the resolution of the experiment, it would be harder to get sufficient growth of the product circle prior to probing.

As discussed in chapter 2 , and in section 2.3 of this chapter, state-selective ionization of DH molecules formed in the reaction is achieved employing Doppler-free $(2+1)$ REMPI using a counterpropagating laser arrangement. The final part of this section will be devoted to a description of the design of the position-sensitive detector that is used to the measure the $\mathrm{DH}^{+}$ions.

The basic principle of the position-sensitive ion detector used, which is shown schematically in Figure 3, is image intensification of a spatial ion distribution by the application of microchannel plates. By using two-stage image intensification, a single ion leads to the formation of a shower of electrons, which, after being accelerated towards a phosphor screen, lead to a photon signal which is strong enough to be recorded by a CCD camera. The signal on the camera is transferred to an image processor. Unlike more conventional ion detectors the application of microchannel plates leaves the spatial information of the ion signal intact, and in the last ten years these devices have become a very valuable tool in physical chemistry experiments ${ }^{21)}$. The position-sensitive ion detector used in the current $\mathrm{D}+\mathrm{H}_{2}$ experiment was the 


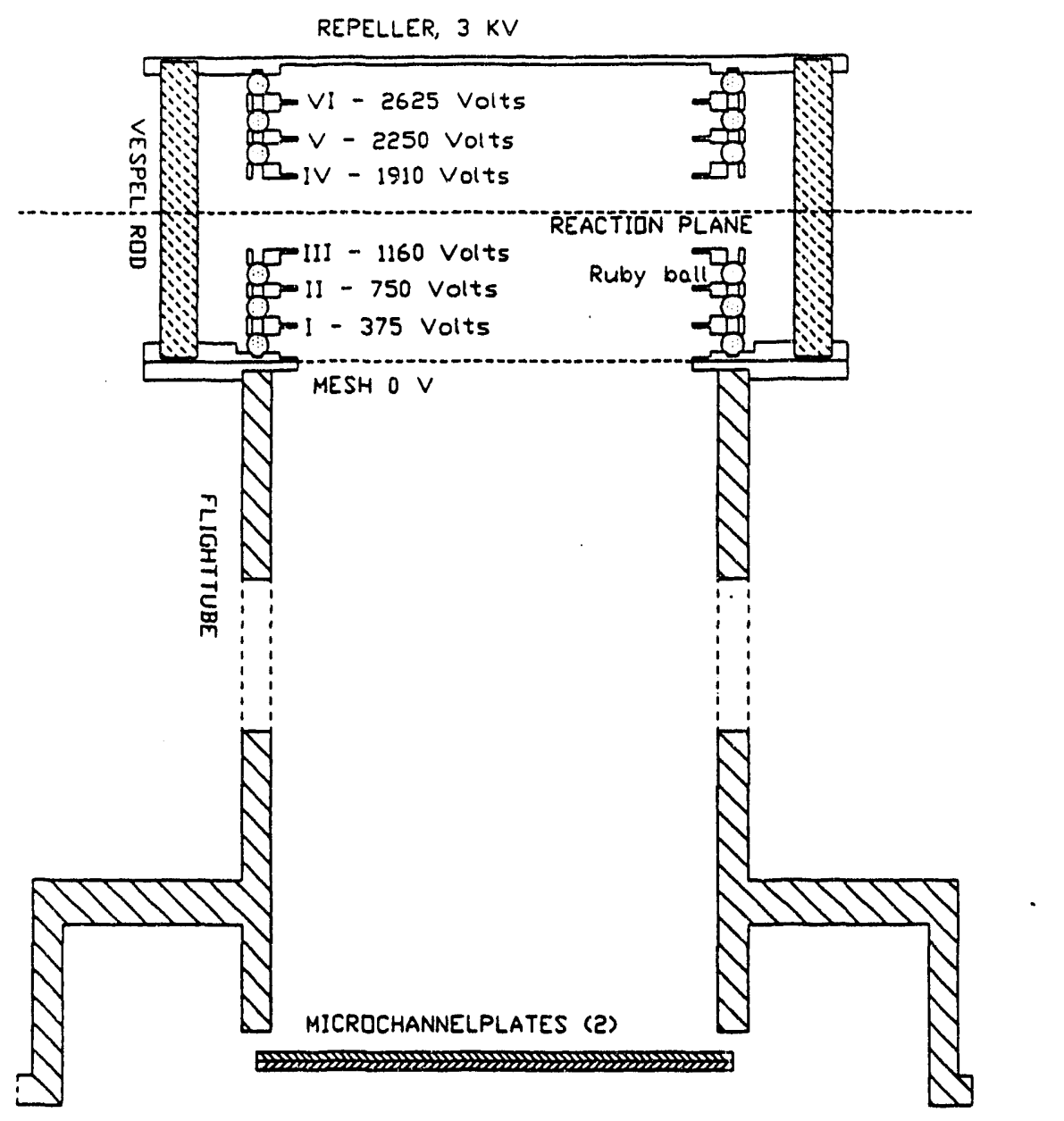

Figure 3 Design of the position-sensitive ion detector used in the $\mathrm{D}+\mathrm{H}_{2}$ experiment. The extraction region consists of a stack of electrodes designed to give a very homogeneous extraction field which leaves the spatial distribution of the ions intact. Through a fine mesh the ions enter a drifttube which provides the time-of-flight separation required for mass-selected ion detection. The ion signal is amplified using two microchannel plates followed by a phosphor screen and detected using a video system described in more detail in the text. 
result of a cooperation with Hamamatsu Photonics, and has several desirable characteristics which set it apart from most position-sensitive ion detectors currently used. First of all, the detector employs time-of-flight separation of the different ion masses and gating of the high voltage which is applied to the microchannel plates enabling an experiment where detection is possible for a single mass. In the $\mathrm{D}+\mathrm{H}_{2}$ experiment this is very crucial, since at the laser powers required for detection of the DH molecules, many background gasses in the main chamber are efficiently ionized, leading to an undesirable ion signal which can easily be six orders of magnitude larger than the $\mathrm{DH}^{+}$signal of interest. Fortunately, this undesirable ion signal appears almost exclusively at higher masses $(m>25)$, and therefore an experiment with gated detection is feasible. The flighttime of the $\mathrm{DH}^{+}$should be long enough to allow for sufficient separation between mass $3\left(\mathrm{DH}^{+}\right)$and masses $2\left(\mathrm{H}_{2}^{+}\right)$and $4\left(\mathrm{D}_{2}{ }^{+}\right)$, respectively, and was chosen to be $500 \mathrm{nsec}$. With this flighttime for mass 3, masses 2 and 4 will have flighttimes of approximately $410 \mathrm{nsec}$ and $580 \mathrm{nsec}$ respectively, and application of a $50 \mathrm{nsec}$ gate on the microchannel plate operation will guarantee detection of mass 3 only. A choice of a flighttime longer than $500 \mathrm{nsec}$ is not only unnecessary, but also undesirable, since the $\mathrm{DH}$ product molecules which are detected near the edge of the detector (i.e. at the largest laboratory frame angles) can have a radial velocity component (directed at the edge of the detector) of up to $5000 \mathrm{~m} / \mathrm{sec}$, corresponding to a $2.5 \mathrm{~mm}$ displacement parallel to the reaction plane during the timeof-flight. With a longer time-of-flight these molecules might fly out of the effective area of the microchannel plates. In the current design the desired $\mathrm{DH}^{+}$flighttime is realized by accelerating the ion to $1.5 \mathrm{kV}$ over a $1 \mathrm{~cm}$ acceleration length, followed by a $13.5 \mathrm{~cm}$ driftlength. At $1.5 \mathrm{kV}$ of kinetic energy the microchannel plates approach their maximum efficiency of ion detection, which is about $60 \%{ }^{22}$. The inicrochannel plates used have a $32 \mathrm{~mm}$ effective area and a channel length-to-diameter ratio of 40 , giving a single stage gain of $10^{4}$ at $900 \mathrm{~V}$ between the cathode and the anode, and a gain of $10^{6}-10^{7}$ for the two stage assembly used in the experiment. In the gated operation the microchannel plates are set up as part of a $50 \Omega$ transmission line, with the cathode held at ground potential and a $50 \mathrm{nsec}$ long pulse of up to $1.8 \mathrm{kV}$ 
travelling over the anode surface. The phosphor screen is held at a voltage of $2.5-3$ $\mathrm{kV}$ with respect to the microchannel plate anode surface.

As the signal calculations in the next sections will show the signal level in the $\mathrm{D}+\mathrm{H}_{2}$ experiment is on the order of a few ions per laser shot and therefore single ion detection capability is absolutely necessary. In most position-sensitive detectors which are in use data is acquired by accumulating images on a CCD camera for a relatively long time (seconds or minutes), and for signal levels as low as the ones in this experiment the CCD camera has to be thermoelectrically or cryogenically cooled, in order to reduce the thermal noise. At the onset of data acquisition a single ion could readily be recognized above the thermal noise on most available camera systems, but, due to the finite resolution of the experiment, successive detected ion peaks are distributed over a finite area, and in time, unless a cooled camera is used, the signal would rapidly be lost $i$ the thermal noise, which is rising uniformly. In the current position-senslive det cooled camera is rendered unnecessary by application of the video microscopy technique ${ }^{23)}$. This is essentially a ion/photon counting imaging technique in which the images on the camera are read and digitized at a relatively high clockrate $(30 \mathrm{~Hz})$ and single ion events can be measured with excellent signal-to-noise of about 100 . In fact, the camera noise is completely filtered out in the digitization process. In order to eliminate cross-talk between the pulsed operation of the microchannel plates and the CCD camera, the experiment and the camera readout can be synchronized.

In the $\mathrm{D}+\mathrm{H}_{2}$ experiment the spatial resolution of the position-sensitive detector need not be extremely high since the velocity component $v_{x}$ perpendicular to the laser axis follows from the accurately known time delay between the photolysis laser and the detection laser, and the corresponding $v_{y}$ from the well-known energetics for the $D$ atom generation and the reaction itself. More specifically, the resolution of the camera needs to allow for a separate measurement of the intensity of the two peaks corresponding to the points where the product circle intersects the laser axis, but the actual position of these peaks on the detector surface is not so crucial. Still, considerable effort was put into designing a position-sensitive detector with good 
Figure $4 \quad$ Results of trajectory calculations performed to optimize the ion extraction electrode geometry used in the $\mathrm{D}+\mathrm{H}_{2}$ experiment. The absolute value of the angular deviation of a $\mathrm{DH}^{+}$ion with no initial velocity is shown for a number of geometries as a function of the radial position in the detection plane. Curves I - III show how the addition of electrodes at intermediate voltages between the repeller and the mesh at the entrance of the drift tube improves the resolution significantly. Further improvement is achieved by increasing the difference between the inner and outer diameter from 4 to $8 \mathrm{~mm}$ (IV) and by shaping the electrodes as shown in Figure 3, i.e. thin at the inner diameter and thick at the outer diameter (VI). Curves I - VI are for extraction geometries where the additional electrodes are evenly spaced between the repeller and the mesh. In the calculation of curves VII and VIII, as in the actual experiment, the gap between the two central electrodes is opened up to $5 \mathrm{~mm}$, and curve VIII shows how in this case the penetration of the ground plane of the $\mathrm{D}+\mathrm{H}_{2}$ source plane geometry can be reduced by changing the voltages on the two central electrodes from those determined based on their geometrical location, as shown in Figure 3. 


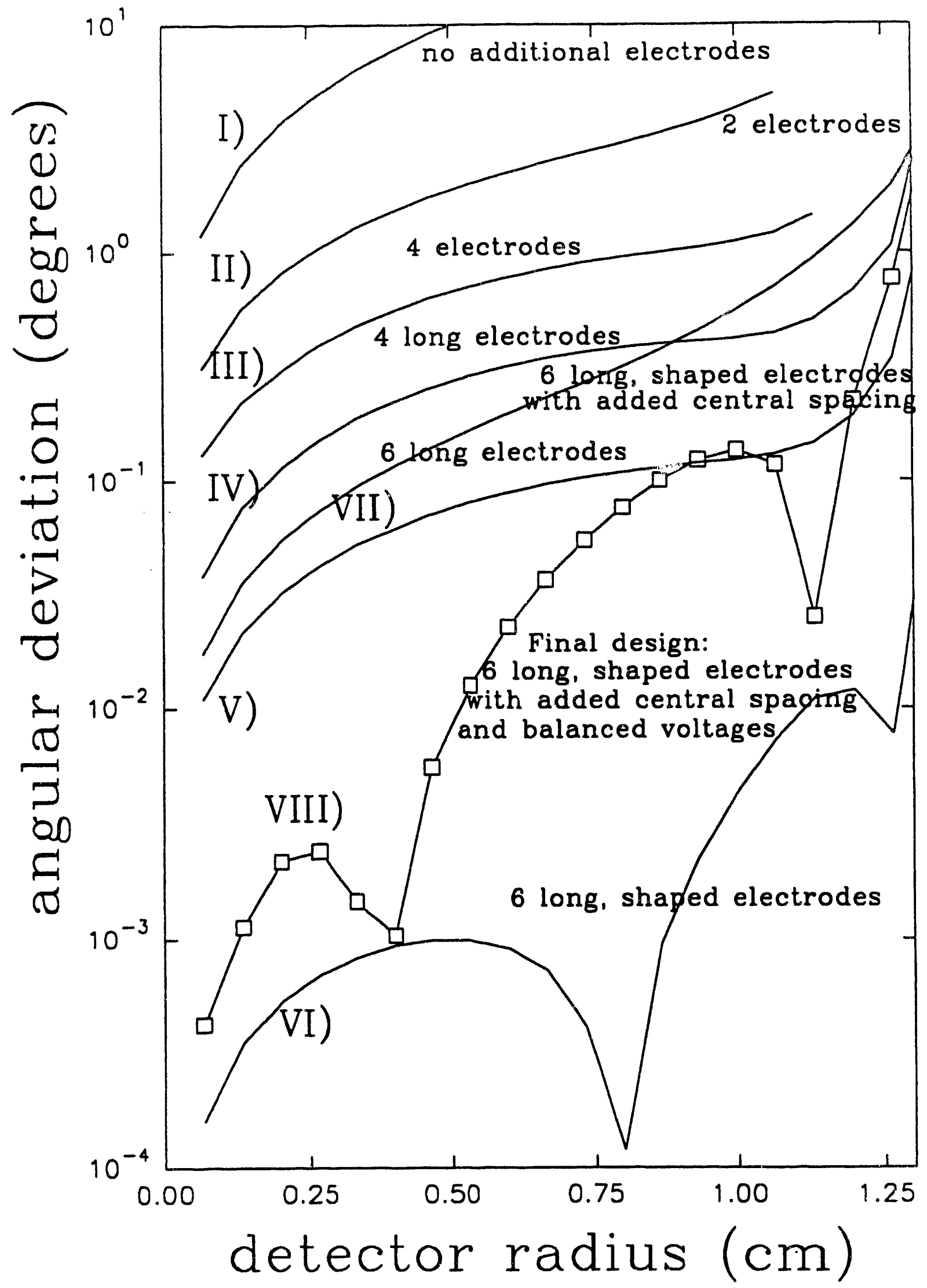




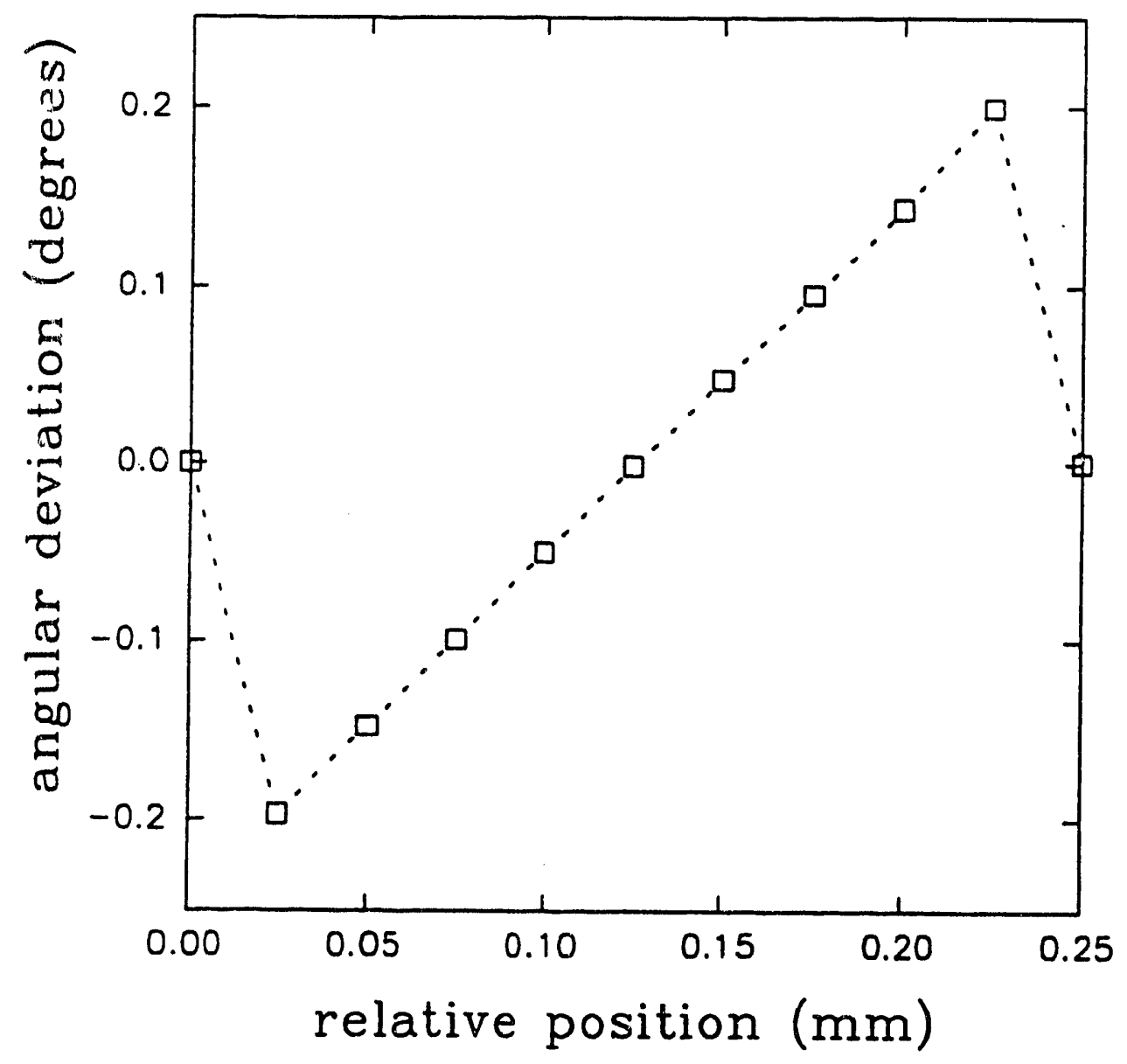

Figure 5 Influence of the passage of the $\mathrm{DH}^{+}$ions through a mesh at the entrance to the drift tube with a $0.25 \mathrm{~mm}$ mesh spacing. The angular deviation of the $\mathrm{DH}^{+}$ion is shown as a function of the position between two consecutive meshwires. 
spatial resolution.

The spatial resolution of the position-sensitive ion detector depends almost exclusively on the quality of the extraction field and perturbations in the ion trajectories as the ion passes from the extraction field to the field-free drift area through a fine wire mesh; the image intensification of the MCP-phosphorscreen assembly plays only a negligible role in the resolution of the detector. In the extraction field the ions are accelerated and acquire a velocity component perpendicular to the plane of reaction. Ideally, the equipotential surfaces of the extraction field run perfectly parallel to the reaction plane, but particularly near the edge of the detector this situation is difficult to realize. In order to shield the acceleration region from surrounding ground voltages in the experimental apparatus, a total of six acceleration electrodes were added between the repeller plate and the fine wire mesh, as shown in Figure 3, and set at intermediate voltages. In order to obtain the best performance these electrodes were shaped in the following way: while on the outside the electrodes are reiatively thick and closely spaced, in order to provide minimal penetration of surrounding voltages, on the inside the electrodes are only $0.5 \mathrm{~mm}$ thick, in order to minimize the range where the potential energy is held constant because of the finite size of the electrode. The space between the two central accelerator electrodes was held rather large at $5 \mathrm{~mm}$, in order to minimize the detrimental effects of scattered light from the high power ultraviolet lasers used for the DH detection. Extensive calculations were performed to optimize the extraction field using charged particle trajectory simulation program SIMION ${ }^{24)}$, the results of which are shown in Figure 4. Due to geometrical constraints in the experiment, the outer diameter of the electrodes is limited to $34 \mathrm{~mm}$, whereas, in the $\mathrm{D}+\mathrm{H}_{2}$ experiment, good extraction is required within the central $20 \mathrm{~mm}$ of the detector. With the extraction geometry shown in Figure 3, extraction is possible where within the central $22 \mathrm{~mm}$ range of the $26.6 \mathrm{~mm}$ ID electrodes the distortions of the trajectories measured at the first microchannel plate are $0.5 \mathrm{~mm}$ or less.

The dominant spatial resolution limiting effect in the current position-sensitive ion detector is the distortion of the ion trajectories acquired when passing through the 
fine wire mesh separating the extraction field from the flight tube. As shown in Figure 5 , the ion trajectories are bent, depending on the location of the ion trajectory with respect to adjacent mesh wires, and a blurring of the overall image takes place. Trajectory calculations indicated that for a $0.25 \mathrm{~mm}$ mesh wire spacing the trajectories açuire a distribution of distortion angles with a FWHM of 0.45 degrees, corresponding to a blurring of the image to $1.1 \mathrm{~mm}$ FWHM at the detector. In the existing detector a $90 \%$ transmitting $\mathrm{Cu}$ mesh with a mesh wire spacing of $0.27 \mathrm{~mm}$ (Buckbee-Mears Co. Part.no. MC-20) is implemented. Clearly the blurring caused by this mesh not negligible but it is small enough compared to the resolution of the experiment and clearly meets the resolution requirements described above. Improvement in the resolution by the use of a mesh with a smaller wire spacing is only possible at the expense of significant ion transmission losses.

The image processing is carried out in the following way: Light emitted from the phosphor screen (P-20) is coupled out of the apparatus through a fiberoptic, and is imaged onto a CCD camera (Hamamatsu model C3077) using a $4.3: 1$ telescope. The camera is interfaced to an image processor (Hamamatsu model DVS-3000), where the signal is accumulated. This image processor, in tum, is interfaced to an IBM PC, where the main $x_{i}$ accumulation and processing occurs.

As discussed in some detail in chapter 2, several precautions were taken to reduce the influence of extraneous signals in the experiment. As pointed out the gating of the microchannel plates plays an important role in eliminating signals from undesired ions. However, this technique only discriminates against ions formed directly by the laser, which are accelerated to a well-defined, uniform kinetic energy. If, as the direct or indirect result of scattered light, an ion is formed at a position outside the reaction plane, its flighttime is modified and may overlap with the $\mathrm{DH}^{+}$ signal of interest. Thus it is important to have the laser beams pass through the chamber without causing too much scattered light. To achieve this, light baffles were placed on the entry and exit tubes of the detection laser and on the entry tube for the photolysis laser. To minimize reflections all laser entrance and exit windows were placed at Brewster's angle. The cross-section for a typical electron impact ionization 
event is about $10^{-16} \mathrm{~cm}^{2}$ ), so at a pressure of $10^{-6} \mathrm{Torr}$, about $310^{-6}$ ions are formed per $\mathrm{cm}$ of electron travel. Therefore, the production of ions from electron impact can be held to a minimum quite easily. Another matter is the detection of the photoelectrons themselves. In the extraction region the electric field directs the electrons away from the entrance of the flighttube, so one need not worry, however, photoelectrons created inside the flighttube are in a field-free area where they can drift for a considerable amount of time before possibly being detected. To inhibit the detection of these photoelectrons, the flighttube is held at a +60 Volts bias with respect to the cathode surface of the microchannel plates.

In initial experiments some extraneous signals could be observed resulting from electrons being formed in the DI photolysis region. Placing an approximately $+100 \mathrm{~V}$ mesh above the reaction plane (facing the DI nozzle) eliminated most of this effect.

Having eliminated the appearance of extraneous signals in the manner described above, the video microscopy technique in combination with the synchronization of the experiment to the camera, secures noise-free signal detection.

\subsection{Yield of reactive product for $\mathrm{D}+\mathrm{H}_{2} \rightarrow \mathrm{DH}+\mathrm{H}$}

In this section a calculation will be presented for the number of DH molecules which can be produced in the crossed molecular beam experiment which was outlined in section 2.1. The calculation resembles a treatment in reference 6 , and proceeds by the following steps. First, an analysis will be presented of the production of $D$ atoms through the photolysis of DI. The transport of $D$ atoms to the interaction region will he discussed, followed by a calculation of the amount of $\mathrm{DH}$ produced when the pulsed beam of $D$ atoms is crossed with a pulsed beam of $\mathrm{H}_{2}$ molecules.

Before going into the details of the calculations it is useful to discuss qualitatively a few limiting factors that will be encountered, that determine the outcome of these calculations to a large extent. First of all, in the photolysis of the DI and the generation of the $D$ atom beam, there will be a limit on the DI pressure in the photolysis volume. As the DI pressure is increased, a point is reached where, prior to 
entering the main chamber, a large fraction of the $D$ atoms undergo an inelastic collision with residual DI or with I atoms. At this point the monochromaticity of the D atom velocity, which is absolutely crucial in this experiment, will be spoiled. A similar effect occurs in the interaction region. As the $\mathrm{H}_{2}$ pressure in the interaction region is increased, a point is reached where the experimental angular distribution of the $\mathrm{DH}$ molecules formed in the reaction will show the influence of velocity-changing secondary collisions with ambient $\mathrm{H}_{2}$ molecules. While both these factors limit the signal which can be obtained in the experiment, it should be noted that they also facilitate reproduction of the kind of conditions under which Continetti et.al. did their successful experiment ${ }^{4,6)}$. The observation of velocity-changing collisions of the $D$ atoms in conjunction with a measurement of the power dependence of the $D$ atom beam intensity is a good method to assess whether a sufficiently intense $D$ atom beam has been established, and observation of secondary collisions of the $\mathrm{DH}$ formed in the reaction or attenuation of the $\mathrm{D}$ atom beam by the $\mathrm{H}_{2}$ beam, can be used as an indicator for the amount of $\mathrm{DH}$ produced in the reaction.

Other limitations encountered in the calculation relate mainly to the resolution which is required in a meaningful experiment. In order to have a well-defined relative velocity vector for the colliding partners there has to be a minimum ratio between the distance from the photolysis volume to the reaction volume on one hand and the size of these volumes on the other. Similarly there is a requirement for the minimum ratio between the size of the product circle as it is probed by the detection laser and the size of the reaction volume.

\section{D atom production}

In the description of the $\mathrm{D}$ atom production multi-photon absorption leading to ionization of the DI molecules will be neglected. The photolysis of the DI molecules is then described by 


$$
\dot{N}=\sigma_{\text {absorption }} F N
$$

where $\mathrm{N}$ is the number of DI molecules in the photolysis volume, $\sigma_{\text {absoption }}\left[\mathrm{cm}^{2}\right]$ is the DI absorption cross section at the photolysis wavelength and $\mathrm{F}\left[\mathrm{cm}^{-2} \mathrm{~s}^{-1}\right]$ is the instantaneous photolysis laser photon flux. Integration over the time duration of the laser pulse gives

$$
N_{\text {des }}=N\left(1-\exp \left[-\sigma_{\text {absorption }} F \Delta T\right]\right)
$$

where $\Delta T$ [sec] is the length of the photolysis laser pulse. If a cubic photolysis laser volume $\mathrm{V}\left[\mathrm{cm}^{3}\right]=l_{0}^{3}\left[\mathrm{~cm}^{3}\right]$ is assumed, the instantaneous photon flux can be written as

$$
\begin{gathered}
F=\frac{E[\mathrm{~J} / \mathrm{pulse}] * 5.0310^{22}\left[\mathrm{~cm}^{-1} / J\right]}{\left(10^{7} / \lambda[\mathrm{mm}]\right)\left[\mathrm{cm}^{-1} / \mathrm{photon}\right] * L_{0}^{2}\left[\mathrm{~cm}^{2}\right] * \Delta T[\mathrm{sec}]} \\
-5.0310^{24} \frac{E[\mathrm{~J} / \mathrm{pulse}] * \lambda[\mathrm{nm}]}{\zeta_{0}^{2}\left[\mathrm{~cm}^{2}\right] * \Delta T[\mathrm{nsec}]}\left[\text { photons } \mathrm{cm}^{-2} \mathrm{sec}^{-1}\right]
\end{gathered}
$$

and the number of $D$ atoms produced is given as

$$
N_{d x s}=n_{0} l_{0}^{3}\left[1-\exp \left(-5.0310^{15} \frac{\sigma_{\text {absorpaton }} E \lambda}{l_{0}^{2}}\right]\right.
$$

where $\mathrm{n}_{0}\left[\mathrm{~cm}^{-3}\right]$ is the density of DI molecules in the photolysis volume. As discussed in the introduction to this section there is an upper limit to the DI density in the photolysis area because the $\mathrm{D}$ atoms can undergo velocity-changing collisions with residual DI molecules or I atoms. Following a Beer's law type argument there is an 
upper limit on the product of the DI density $\mathrm{n}_{0}$ and the photolysis volume width $l_{0}$. Continetti determined experimentally that a reasonable $D$ atom beam quality is obtained as long as ${ }^{6}$

$$
n_{0} * l_{0} \leq 2.410^{14}\left[\mathrm{~cm}^{-2}\right]
$$

Using the upper limit defined by this relation, Equation 6 changes to

$$
N_{\text {des }}-2.410^{14} l_{0}^{2}\left[1-\exp \left(-5.0310^{15} \frac{\sigma_{\text {aboorpdon }} E \lambda}{l_{0}^{2}}\right]\right.
$$

It is instructive to look at the behaviour of Equation [8] for large and small values of the photolysis area width $l_{0}$. For small values of $l_{0}$, the exponential term in Equation [8] vanishes and the numver of $D$ atoms produced scales with $l_{0}^{2}$. In a small photolysis volume the DI photolysis is readily saturated and the number of $D$ atoms produced is given by the number of DI molecules in the photolysis volume $\mathrm{N}=\mathrm{n}_{0} l_{0}{ }^{3}$, under the restriction that $n_{0} l_{0}$ does not exceed the maximum defined by Equation [7], and therefore effectively an $l_{0}^{2}$ dependence follows. For large values of the photolysis area width, the exponential term in Equation [8] can be Taylor-expandecì as

$$
\exp \left(-5.0310^{15} \frac{\sigma_{\text {absopption }} E \lambda}{l_{0}^{2}}\right) \sim 1-5.0310^{15} \frac{\sigma_{\text {ebsoppton }} E \lambda}{l_{0}^{2}}
$$

and the number of $\mathrm{D}$ atoms produced becomes independent of the width of the photolysis area as

$$
N_{\text {dis }}-1.210^{30} \sigma_{\text {ebsoopdion }} E \lambda
$$

Again in this case the total number of molecules in the photolysis volume equals $\mathbf{N}=$ $\mathrm{n}_{0} l_{0}^{3}$ with the restriction that $\mathrm{n}_{0} l_{0}$ does not exceed the maximum defined in Equation [7], but in this case, where only a small fraction of the DI molecules are dissociated, the effective $l_{0}^{2}$ increase in the number of DI molecules is offset by the fact that the 


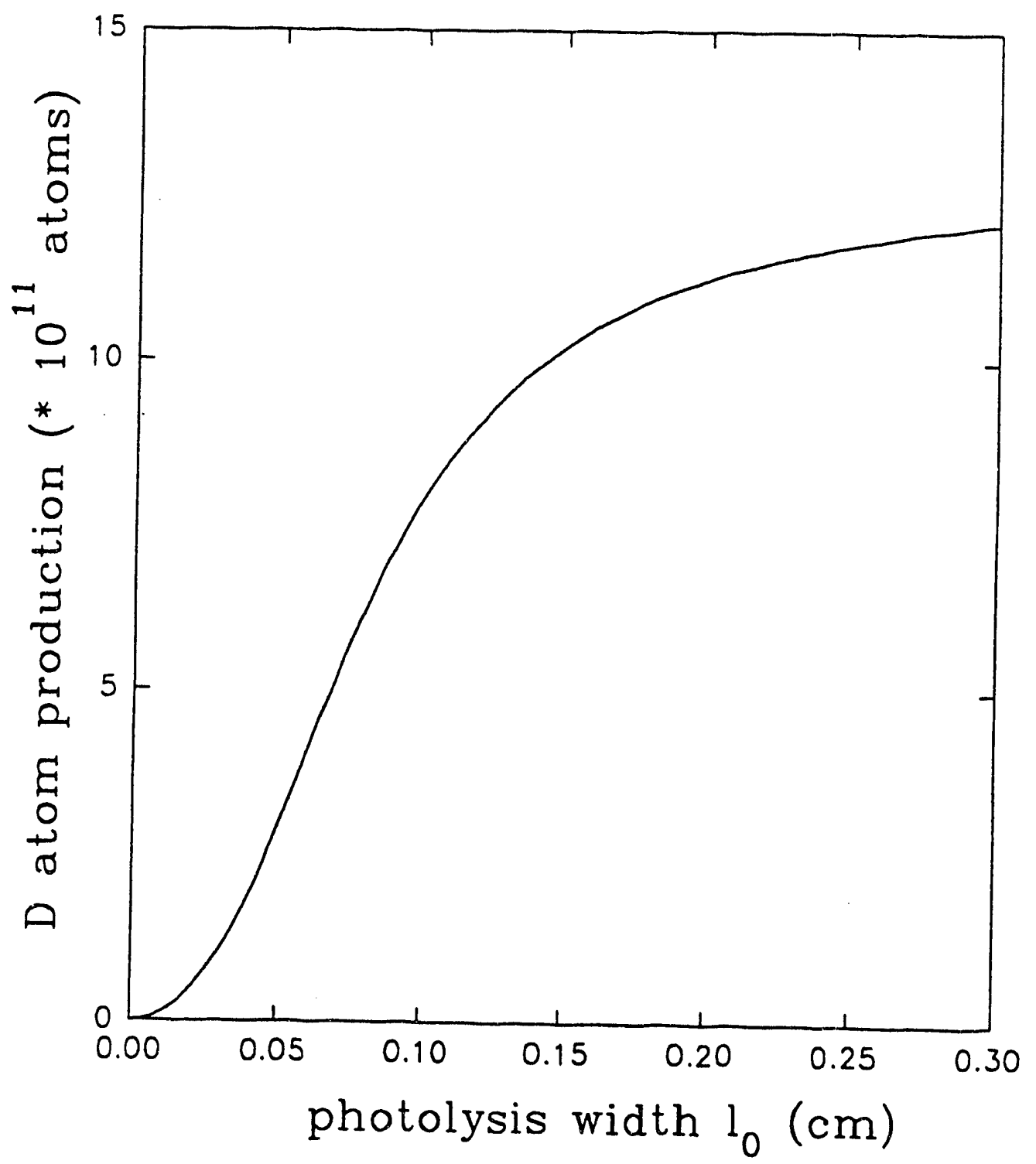

Figure 6 Dependence of the $\mathrm{D}$ atom production on the width of the photolysis area, calculated for a laser power of $50 \mathrm{~mJ} /$ pulse at $266 \mathrm{~nm}$. 
photonflux is inversily proportional to $l_{0}^{2}$.

In their experiments, Continetti et. al. focussed $110 \mathrm{~mJ} /$ pulse of $248 \mathrm{~nm}$ radiation into a $3 \mathrm{~mm}$ wide photolysis volume ${ }^{4,6)}$. This corresponded to a condition where about $45 \%$ of the DI molecules in the photolysis volume were dissociated. As discussed later, the detection scheme in the current experiment requires the use of a significantly smaller photolysis volume and therefore, although the photolysis laser powers used are much lower, the fraction of molecules that are dissociated tends to be higher.

In considering the number of $\mathrm{D}$-atoms that can be used in the experiment it is necessary to substract the $\mathrm{D}$ atoms which undergo velocity-changing collisions before leaving the photolysis volume from the numbers calculated above. If we assume that on average the $\mathrm{D}$ atom has to travel a distance $l_{0} / 2$ in order to leave the photolysis volume, then, using a quenching cross section $\sigma_{\text {quench }}$ of $3510^{-16} \mathrm{~cm}^{26}$ ) the fraction of D atoms that leave the photolysis volume without secondary collisions is given as

$$
f_{\text {upperturbed }} \sim \exp \left[-n_{0} l_{0} \sigma_{\text {quanch }} / 2\right]-0.65
$$

In addition the two polarizations of the DI absorption have to be taken into account. At a wavelength of $266 \mathrm{~nm}$ the ratio between the partial cross sections for the perpendicular and parallel transitions is about $3: 1^{16)}$, so about $75 \%$ of the $\mathrm{D}$ atoms are produced with the correct velocity. In conclusion, about $50 \%$ of the D-atoms that are truly available for use in the subsequent experiments.

Choosing as the photolysis laser the 4th harmonic of a Nd:YAG laser at 266 $\mathrm{nm}$, where $\sigma_{\text {absorption }}$ is equal to $1.610^{-19} \mathrm{~cm}^{2}$ 27), and performing the calculation with a laser pulse energy of $50 \mathrm{~mJ} /$ pulse, Figure 6 shows the dependence of the number of $D$ atoms produced as a function of the width of the photolysis area $l_{0}$. Under typical experimental conditions about $7.510^{11}$ useful $\mathrm{D}$ atoms can be produced per lasershot. 
D atom transport to the interaction region and calculation of reactive product

The angular distribution for direct dissociation of a diatomic molecule via a perpendicular transition is given $b^{28)}$

$$
I(\theta)-\frac{1}{4 \pi}\left[\frac{3}{2}-\frac{3}{2} \cos ^{2} \theta\right]
$$

where $\theta$ is the angle between the laser polarization axis and the velocity vector of the recoiling photofragments, which is $\pi / 2$ in the current experiment. If a cubic interaction region $\mathrm{V}\left[\mathrm{cm}^{3}\right]=l_{13}\left[\mathrm{~cm}^{3}\right]$ and a distance $d_{1}[\mathrm{~cm}]$ between the photolysis region and the interaction region are assumed, then the solid angle subtended by any point in the DI photolysis volume can be approximated by

$$
\Delta \Omega-\left[\frac{l_{1}}{d_{1}}\right]^{2}
$$

and the number of $D$ atoms passing through the interaction region is given by

$$
N_{D, \text { warection }}=N_{D, \text { phowotysts }} \frac{3}{8 \pi}\left[\frac{l_{1}}{d_{1}}\right]^{2}
$$

As will be shown later, under typical conditions, about $0.12 \%$ of the $D$ atoms produced in the photolysis volume will pass through the interaction region.

In the interaction region the $\mathrm{D}$ atoms undergo reactive and (in)elastic collisions with the $\mathrm{H}_{2}$ molecules

$$
\begin{aligned}
& D(\vec{u})+H_{2}(v, J) \rightarrow D H\left(v^{\prime}, J^{\prime}, \vec{u}^{\prime}, \theta^{\prime}\right)+H \\
& D(\vec{u})+H_{2}(v, J) \rightarrow D\left(\vec{u}^{\prime}\right)+H_{2}\left(v^{\prime}, J^{\prime}\right)
\end{aligned}
$$


and the DH molecules formed in the reaction can undergo further collisions with the $\mathrm{H}_{2}$ molecules as well

$$
D H\left(v^{\prime}, J^{\prime}, \vec{u}^{\prime}, \theta^{\prime}\right)+H_{2} \rightarrow D H\left(v^{\prime \prime}, J^{\prime \prime}, \vec{u}^{\prime \prime}, \theta^{\prime \prime}\right)+H_{2}
$$

Continetti ${ }^{6)}$ concluded that the latter process can best be described hy a hard-sphere cross section of about $1210^{-16} \mathrm{~cm}^{2}$, and determined experimentally that reliable angular distributions for the $\mathrm{DH}$ product molecules could be determined as long as

$$
n_{H_{2}} * l_{1} \leq 310^{13}\left[\mathrm{~cm}^{-2}\right]
$$

Assuming that on average a $\mathrm{DH}$ molecule formed in the reaction has to travel a distance $l_{1} / 2$ in order to leave the interaction region, Equation [17] corresponds to a condition where $1.8 \%$ of the DH molecules formed in the reaction undergo a hardsphere collision with ambient $\mathrm{H}_{2}$ molecules.

The perpendicular component of photolysis of DI at $266 \mathrm{~nm}$ leads to formation of $D$ atoms with a kinetic energy of $1.54 \mathrm{eV}$. The $\mathrm{H}_{2}$ velocity is about $2930 \mathrm{~m} / \mathrm{sec}$ $\left(\mathrm{E}_{\text {kin }}=0.090 \mathrm{eV}\right)$, and therefore the collision energy at this photolysis wavelength is $0.81 \mathrm{eV}$. At this collision energy the reactive cross section is equal to $0.8110^{-16} \mathrm{~cm}^{2}$ 29) and, under the restriction set by Equation [17], the fraction of $D$ atoms that react to form a $\mathrm{DH}$ molecule is given by

$$
f_{\text {recocion }}-1-\exp \left[-n_{H_{2}} l_{1} \sigma_{\text {recciton }}\right] \sim 0.0024
$$

By combining the equations from this section one can now write down the following equation for the total number of DH molecules produced per lasershot 


$$
\begin{gathered}
N_{D H}=n_{0} l_{0}^{3}\left[1-\exp \left(-5.0310^{15} \frac{\sigma_{\text {aboorpton }} E \lambda}{l_{0}^{2}}\right)\right] * \\
{\left[\frac{I_{1}}{I_{\perp}+I_{l}}\right] \exp \left(-n_{0} l_{0} \sigma_{q \text { qunch }} 2\right) *} \\
\frac{3}{8 \pi}\left[\frac{l_{1}}{d_{1}}\right]^{2}\left[1-\exp \left(-n_{H_{2}} l_{1} \sigma_{\text {recoction }}\right)\right]
\end{gathered}
$$

with the dimensions of the parameters as defined in the text, and the restrictions on $\mathrm{n}_{0}$, $l_{0}, \mathrm{n}_{\mathrm{H} 2}$, and $l_{1}$ as defined by Equations [7] and [17]. To obtain a minimum acceptable resolution for the experiment one might choose a geometry where $l_{0}=l_{1}=d_{1} / 10$. Under these conditions it is observed that with a choice of $l_{0}=1 \mathrm{~mm}$ about $7.510^{11}$ useful D atoms can be generated per lasershot. About $0.12 \%$ of these D atoms cross the interaction region, and of these atoms about $0.24 \%$ will undergo reaction, leading to a total of about $2.210^{6} \mathrm{DH}$ molecules formed per lasershot. These $\mathrm{DH}$ molecules are distributed over a number of ro-vibrational quantum states and in order to be able to do a useful experiment we will make it our goal to be able to detect the angular distribution for a state that carries at least $2 \%$ of the population. To measure the angular distribution we need to let the product globe expand prior to detection. Assuming that a minimal globe diameter $\mathrm{d}_{\mathrm{g}}=10 l_{0}$ is required to obtain a minimum acceptable resolution in the experiment, and assuming at this moment for simplicity that the distribution of DH molecules on the product globe is homogeneous, a detection technique is required that is capable of detecting a surface density

$$
\sigma_{D H, \min }=\frac{N_{D H\left(N_{N}\right), \operatorname{mm}}}{\pi d_{g}^{2}} \sim 1.410^{4}\left[\text { molecules } \mathrm{cm}^{-2}\right]
$$

As will be demonstrated in the next section, the only way to detect this DH surface density is by application of the Doppler-free $(2+1)$ REMPI technique discussed in chapter II. 


\subsection{Laser detection of reaction products from $\mathrm{D}+\mathrm{H}_{2} \cdots \mathrm{DH}+\mathrm{H}$}

In the previous section the conclusion was reached that if a mearingful crossed molecular beam experiment for the reaction of $\mathrm{D}+\mathrm{H}_{2}-\mathrm{DH}+\mathrm{H}$ with positionsensitive detection of molecular reaction product is to be carried out, a detection technique is required which is capable of probing the globe on which all the product molecules lie when the product molecule surface density equals approximately $1.410^{4}$ [molecules $\mathrm{cm}^{-2}$ ]. In this section it will be shown that this requirement is met by the Doppler-free $(2+1)$ REMPI technique discussed in chapter II.

The $\mathrm{D}+\mathrm{H}_{2}$ experiment discussed in this chapter is an excellent example of the situation discussed in chapter II, where a chemically interesting system displays a very large effect: ve Doppler width. In the $\mathrm{D}+\mathrm{H}_{2}$ experiment there is a Doppler width of up to $3 \mathrm{~cm}^{-1}$ associated with the motion of the product molecules with respect to the center-of-mass velocity, which cannot be compensated for by choosing any particular laser geometry in a detection scheme that relies on a single-photon resonance and this makes it impossible to use the $(1+1)$ REMPI technique, as we will now proceed to show in more detail.

As mentioned in chapter II the highest sensitivity reported for $(1+1)$ REMPI of hydrogen is $10^{4}$ molecules/cc for molecules with a Dopplershift within the bandwidth of a near transform-limited $210 \mathrm{MHz}$ FWHM xuv laser ${ }^{30}$. With a Doppler width of 3 $\mathrm{cm}^{-1}$ (90 Ghz) one would need

$$
-10^{4} \frac{\text { molecules }}{\mathrm{cm}^{3} \text { state }} * \frac{90 \mathrm{GHz}}{0.21 \mathrm{GHz}}-4.310^{6} \frac{\text { molecules }}{\mathrm{cm}^{3} \text { state }}
$$

in order to obtain a detectable signal which is clearly out of reach. One could present the argument that in the current experiment product molecules having the same velocity vector are spacially grouped together. If the detection laser has a diameter $d_{\text {laser }}$ at the intersection with the product globe, then the Doppler width associated with the spread in velocities within the laser volume is given by 


$$
\Delta v=v_{0}\left[\frac{u_{D H_{\text {recoul }}}}{c}\right]\left[\frac{2 d_{\text {laser }}}{d_{8} \tan (\alpha)}\right]
$$

where $\mathrm{u}_{\mathrm{DH}}$ is the recoil velocity of the $\mathrm{DH}$ molecule in the center-of-mass frame and $\alpha$ is defined as the angle between the laser and the product globe. Choosing $d_{\text {laser }}$ to be $0.2 \mathrm{~mm}$ Equation [22] shows that at $\alpha=45^{\circ}$ the Doppler width is reduced by a factor of 25 to about $3.6 \mathrm{GHz}$, requiring only about $1.710^{5}$ molecules $/ \mathrm{cm}^{3}$. In section 2.2 it was found that about $4.410^{4}$ molecules/lasershot will be produced in a product state that accounts for $2 \%$ of the total reaction product. With this product distributed in some fashion on a $1 \mathrm{~cm}$ diameter product globe, the situation is equivalent to having a density of $4.410^{4}$ molecules $/ \mathrm{cm}^{3}$, which therefore is beyond the sensitivity of the detection scheme. Indeed, the only way in which $(1+1)$ REMPI detection would be sensitive enough to detect the $\mathrm{DH}$ products formed in the reaction, would be by probing the product molecules before they have spread out spatially, thus increasing the density of molecules having similar Doppler shifts. The most favourable approach here would be to the crossing region directly. With a $1 \mathrm{~mm}^{3}$ interaction region the local density of molecules in a given ro-vibrational product state is calculated to be $4.410^{7}$ molecules $/ \mathrm{cm}^{3}$, which is feasible given the detection limit of $4.310^{6}$ molecules $/ \mathrm{cm}^{3}$ that applies in this situation. In the current experiment it was chosen not to use this technique, since measuring an angular distribution would require scanning the xuv laser and there was concern that the experimental angular distributions might be influenced by space charge effects, resulting from background molecules ionized by the uv ionization laser. An alternative technique would be to use the Doppler shift to extract the angular information, a technique which obviously is not influenced by space charge. In the Zare group at Stanford aitempts are currently under way to measure $\mathrm{H}_{2}$ angular distributions resulting from photodissociation, by using a $(1+1+1)$ REMPI scheme, using the B- and E- states as the resonant intermediates ${ }^{31)}$. In this scheme the extreme ultra-violet $X \rightarrow B$ transition and the infrared $B \rightarrow E$ transition (which is pumped with a laser which is set up perpendicular to the xuv laser) provide two Doppler shifts from which the $\mathrm{H}_{2}$ velocity 
vector can be determined.

In order to discuss the applicability of Doppler-free $(2+1)$ REMPI to the D + $\mathrm{H}_{2}$ experiment we briefly reiterate the main conclusions from the previous chapter. It was shown that if two $7 \mathrm{nsec}$ long $0.5 \mathrm{~mJ} /$ pulse counterpropagating laser beams were focussed down to a $300 \mu \mathrm{m}$ waist, then in the absence of Stark broadening about $45 \%$ of the molecules in the detection volume would be ionized, corresponding to a single shot detection sensitivity of $1.310^{3}$ molecules/cc for $\mathrm{DH}(\mathrm{v}=0)$. Experimentally some Stark broadening was observed and this accounted for the experimental detection limit of $6.810^{3}$ molecules/cc. Because of the finite quantum efficiency of the microchannel plates used, and transmission losses through the mesh separating the extraction region from the drift tube, the experimental detection efficiency was found to be $1.210^{4}$ molecules/cc in this experimental set-up, or about $5 \%$ of the molecules in the detection volume.

In section 2.2. it was calculated that for a quantum state that carried $2 \%$ of the population, the average surface density on the product globe at the time of detection would be $1.410^{4}$ molecules $/ \mathrm{cm}^{2}$. For a practical situation where $d_{g} \gg d_{\text {laser }}$ the molecules that can be detected by the laser have to within a detector laser radius $d_{\text {lase }} / 2$ from the midplane of the experiment and of these molecules on average a fraction $d_{l a s e} / d_{g}$ will lie in the viewline of the laser. With a $5 \%$ detection efficiency this then leads to the prediction that, on average

$$
1.410^{4} * 0.05 * \pi d_{d} d_{\text {beor }} * \frac{d_{\text {laver }}}{d_{8}}-2.210^{3} d_{\text {laver }}^{2}=2
$$

ions would be formed per lasershot, which, in the absence of any background ions, would certainly be sufficient to do a good experiment.

At this point it is appropriate to spend a few words on the estimated reliability of the results calculated above. As far as the parameters for the crossed molecular beam arrangement are concerned, it is not expected that there are major deviations between the calculations and what can be achieved experimentally. The crossed molecular beam arrangement is very similar to the arrangement used by Continetti et. 
al. ${ }^{4.6)}$ and the levels of DH production in his experiment were succesfully reproduced in a calculation similar to the one given in section 2.2. Thus the results of section 2.2 are expected to be reasonably accurate. The $\mathrm{DH}$ detection efficiency using the Doppler-free $(2+1)$ technique is an experimental result and should be quite accurate as well. While the signal level calculated is not large, the conclusion is that with the proposed technique it should be possible to measure a reasonably accurate angular distribution in no more than a few hours.

Sofar the calculations presented in this section and in the previous one have been primarily applied to the situation of an approximately $1 \mathrm{~mm}$ diameter photolysis area, with all other values related to this one through arguments pertaining to the minimum resolution of the experiment. We are now in a position to further justify this choice. Combining Equations [19] and [20], and using the theoretical (2+1) REMPI efficiencies described by Equations [15] and [20] from chapter 2, the following equation can be written for the average number of detected ions per lasershot, assuming that the ionization of the two-photon excited state is saturated:

$$
\begin{aligned}
& N_{D H(\nu, A)}^{+}-n_{0} l_{0}^{3}\left[1-\exp \left(-5.0310^{15} \frac{\sigma_{\text {abcorpstan }} E \lambda}{\zeta_{0}^{2}}\right)\right] * \\
& {\left[\frac{I_{\perp}}{I_{\perp}+I_{1}}\right] \exp \left(-n_{0} l_{0} \sigma_{\text {qunct }} / 2\right) \quad *} \\
& \frac{3}{8 \pi}\left[\frac{l_{1}}{d_{1}}\right]^{2}\left[1-\exp \left(-n_{H_{2}} l_{1} \sigma_{\text {mection }}\right)\right] f(v, J)\left[\frac{1}{\pi d_{g}^{2}}\right] \pi d_{\text {laser }}^{2}
\end{aligned}
$$

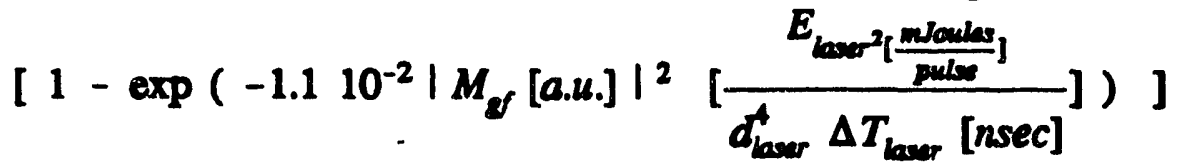

Inspection of Equation [24] shows that, as long as $d_{8} \gg d_{\text {lese }}$ the optimization of the diameter of the detection laser does not depend parametrically on any dimensions for the crossed molecular beam experiment. Thus, having achieved the optimum conditions for the detection, we can now optimize the experimental geometry. According to Equation [24], when comparing geometries that give a constant experimental resolution according to 


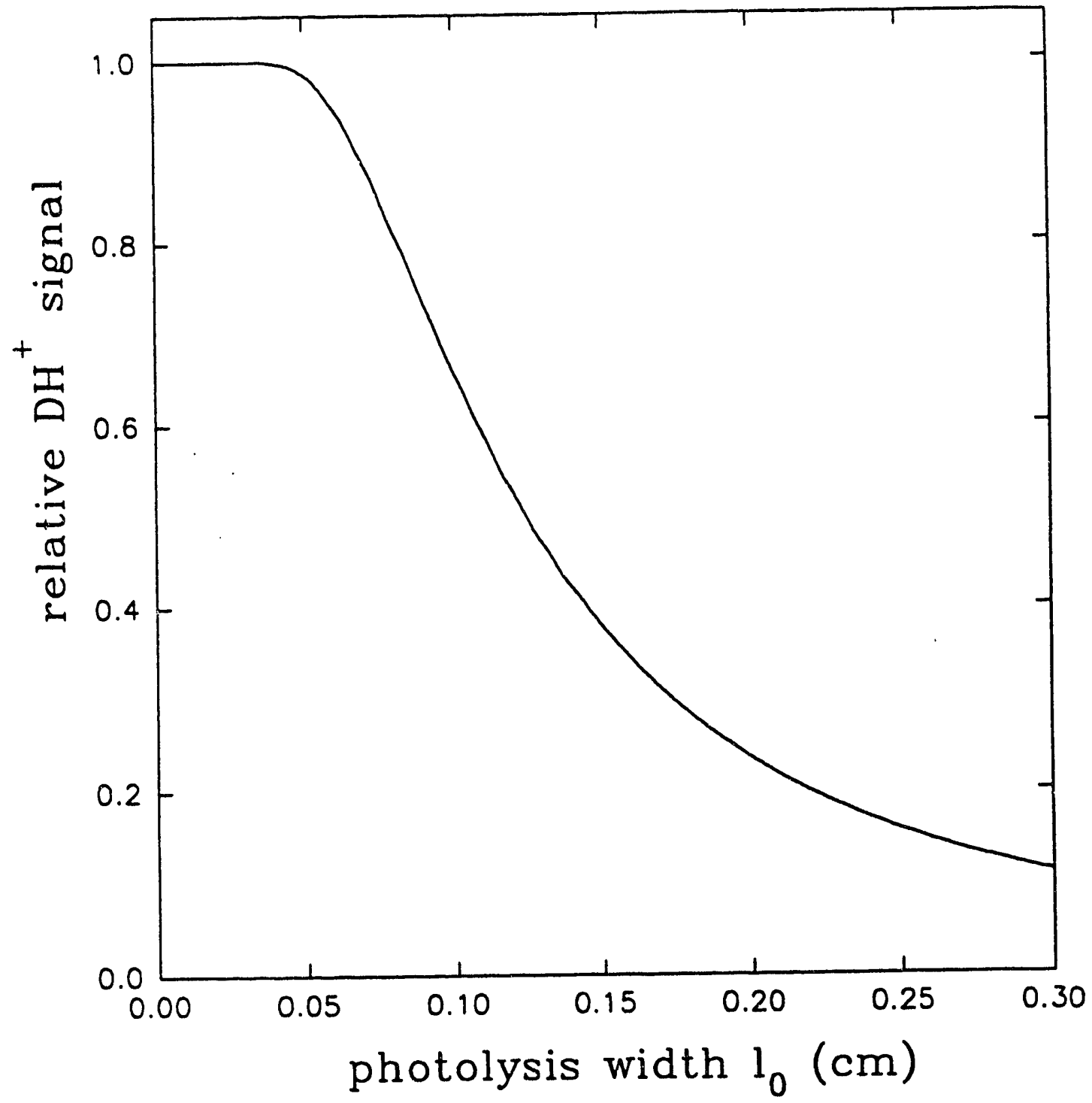

Figure 7 Dependence of the $\mathrm{DH}^{+}$signal on the width of the photolysis area, where all dimensions in the experiment have been scaled in a constant ratio to that width. See text for details. 


$$
l_{0}=c * d_{1}=c^{\prime} l_{1}-c^{\prime \prime} d_{g}
$$

(where typically $c^{\prime} \approx 1$ and $c \approx c^{\prime \prime} \approx 0.1$ ) the obtained ion signal scales with $l_{0}$ as follows:

$$
\begin{aligned}
N_{D H\left(V_{-}\right)}^{+} & -n_{0} l_{0}^{3}\left[1-\exp \left(-\frac{\beta}{l_{0}^{2}}\right)\right] \exp \left(-n_{0} l_{0} \sigma_{\text {quenck }} / 2\right)\left[\frac{c l_{0}}{c^{\prime} l_{0}}\right]^{2}\left[\frac{c^{\prime \prime}}{l_{0}^{2}}\right] \\
& \sim\left[1-\exp \left(-\frac{\beta}{l_{0}^{2}}\right)\right]
\end{aligned}
$$

where the restrictions on $\mathrm{n}_{0}$ and $l_{0}$, and $\mathrm{n}_{\mathrm{H} 2}$ and $l_{1}$ (Equations [7] and [17]) have been applied. Using the values for the photolysis laser power and wavelength and the absorption cross section given below Equation [11], Figure 7 shows that the amount of signal stays nearly constant for values of $l_{0}$ below $0.5 \mathrm{~mm}$, and begins to drop rapidly as $l_{0}$ exceeds $1 \mathrm{~mm}$. This behaviour for small values of $l_{0}$ reflects the offsetting effects of the $l_{0}^{2}$ increase in the production of $\mathrm{D}$ atoms and the $l_{0}^{-2}$ decrease in the density of product molecules in the detection laser volume. As the size of the photolysis region, and thus the entire experiment, becomes bigger, the photolysis will no longer be saturated and the $D$ atom production levels off, whereas the detection efficiency retains the same $l_{0}^{-2}$ dependence, leading to a reduction in signal. In the design of the current experiment $l_{0}$ was chosen to be $1 \mathrm{~mm}$ (i.e. the crossed molecular beam arrangement was constructed with a distance from the photolysis region to the interaction region of $10 \mathrm{~mm}$ ), since significant further reduction in the size of the experimental geometry would significantly have increased the difficulty of constructing the experimental arrangement, and Figure 7 shows that the amount of signal gained would not have been very large.

\section{$\underline{\text { 3. Conclusions }}$}

In this chapter a description has been given of the design philosophy and construction of a new crossed molecular beam experiment to measure angular distributions for the hydrogen exchange reaction. It has been demonstrated how the combination of a 
Doppler-free $(2+1)$ REMPI technique employing the use of a counterpropagating laser beam arrangement and a position-sensitive ion detector allows a determination of the fully state-resolved differential cross sections under single collision conditions. It has been shown how signal calculations for the production and the detection of the product molecules determine the experimental geometry and the details of the application of the laser scheme.

Though the feasability of the experimental scheme was only determined in this chapter for the hydrogen exchange reaction, it is reasonable to expect that application of this scheme to other reactions may be possible as well. Extension of the technique to other hydrogen atom reactions resulting in the formation of molecular hydrogen should be almost trivial, but one need not necessarily stop here. There seem to be two main requirements that a reaction needs to satisfy in order to be suitable for this technique. First of all, it is necessary that the reaction is initiated by a proces involving a laser, in order to meet the timing requirements in the position-sensitive detection scheme. Most often this would be a photolysis proces, like in the current experiment, but one could also conceive an experiment with an optically pumped metastable species or with vibrationally excited reagents. More often than not the deciding factor will be the second requirement, and that is that there is a REMPI scheme (and preferably, a $(2+1)$ REMPI scheme) available for the detection of reaction products. Though more REMPI schemes are rapidly becoming available, this requirement currently limits this technique to a relatively small number of molecules. 


\section{REFERENCES}

1) For a recent review, see W.H. Miller, Ann. Rev. of Phys. Chem. $\underline{41}, 245$ (1990).

2) a) P. Siegbahn and B. Liu, J. Chem. Phys. $\underline{68}, 2457$ (1978).

b) D.G. Truhlar and C.J. Horowitz, J. Chem. Phys. $\underline{68}, 2466$ (1978), $\underline{71}$, 1514(E) (1979).

3) For a recent review of experimental work on the hydrogen exchange reaction, see J.J. Valentini and D.L. Phillips, in "Advances in Gas-Phase Photochemistry and Kinetics - Bimolecular Collisions", edited by M.N.R. Ashfold and J.E. Baggott, (1989), p. 1-51.

4) R.E. Continetti, B.A. Balko and Y.T. Lee, J. Chem. Phys. 93, 5719 (1990).

5) See, for example, W. Demtrodder, "Laser Spectroscopy", Springer (1981).

6) R.E. Continetti, PhD thesis, University of California at Berkeley (1989).

7) a) J.-C. Nieh and J.J. Valentini, Phys. Rev. Lett. 60 , 519 (1988).

b) J.-C. Nieh and J.J. Valentini, J. Chem. Phys. 22, 1083 (1990).

8) a) E. Pollak and M.S. Child, Chem. Phys. 60 , 23 (1981).

b) E. Pollak, J. Chem. Phys. 76, 5843 (1982).

9) J.Z.H. Zhang and W.H. Miller, Chem. Phys. Lett. 153, 465 (1988).

10) a) D.A.V. Kliner, D.E. Adelman and R.N. Zare, J. Chem. Phys. 94,1069 (1990).

b) W.J. van der Zande, R. Zhang and R.N. Zare in "Spectral Line Shapes" (American Institute of Physics, New York, 1990) Vol. 6, p. 301.

11) a) R.E. Continetti, J.Z.H. Zhang and W.H. Miller, J. Chem. Phys. 93 , 5356 (1990).

b) W.H. Miller and J.Z.H. Zhang, J. Phys. Chem. 95, 12 (1991).

12) M. V. Berry, Proc. R. Soc. London A $\underline{392,} 45$ (1984).

13) G. Herzberg and H.C. Longuet-Higgins, Discuss. Faraday Soc. 35,77 (1963).

14) C.A. Mead and D.G. Truhlar, J. Chem. Phys. 70, 2284 (1979).

15) a) B. Lepetit, Z. Peng and A. Kuppermann, Chem. Phys. Lett. 166, 572 
(1990).

b) B. Lepetit and A. Kuppermann, Chem. Phys. Lett. 166, 581 (1990).

c) Yi-Shuen Wu, A. Kuppermann and B. Lepetit, Chem. Phys. Lett. 186, 319 (1991).

16) R.N. Zare, private communication.

17) a) L. Schnieder, K. Seekamp-Rahn, F. Liedeker, H. Steuwe and K.H. Welge, to be published.

b) K.H. Welge, private communication.

18) D. Proch and T. Trickl, Rev. Sci. Instrum. 60, 713 (1989).

19) K.P. Huber and G. Herzberg, "Constants of Diatomic Molecules", Van Nostrand (1979).

20) Ch. Moore (ed.), "Atomic Energy Levels", Nat. Stand. Ref. Data Ser., Nat. Bur. Stand. 35/V (1971).

21) See, for example

a) D.P. de Bruyn and J. Los, Rev. Sci. Instrum. 53, 1020 (1982).

b) D.W. Chandler, J.W. Thoman, M.H.M. Janssen and D.H. Parker, Chem. Phys. Lett. 156, 151 (1989).

c) L. Huewel, A.M. Wodtke, P. Andresen and H. Voges, AIP Conf. Proc., 205 (Phys. Electron. At. Collisions), 337 (1990).

22) Hamamatsu Photonics MCP Assembly, Technical Information, (sept. 1991).

23) R.A. Wick, BioTechniques I. 262 (1989).

24) D.A. Dahl and J.E. Delmore, Idaho National Engineering Laboratory (1987).

25) Y.T. Lee, in "Atomic and Molecular Beam Methods", Vol. 1, edited by G. Scoles, Oxford University Press (1988), p. 554.

26) R.D. Clear, S.J. Riley and K.R. Wilson, J. Chem. Phys. 63, 1340, (1975).

27) J.F. Ogilvie, Trans. Farad. Soc. 67, 2205 (1971).

28) See, for example, R. Bersohn, in "Advances in Gas-Phase Photochemistry and Kinetics - Molecular Photodissociation Dynamics", edited by M.N.R. Ashfold and J.E. Baggott, (1987), p. 1-30.

29) D.L. Phillips, H.B. Levene and J.J. Valentini, J. Chem. Phys. 90, 1600 (1989). 
30) E.F. Cromwell, $\mathrm{PhD}$ thesis, University of California at Berkeley, (1991).

31) Neil Shafer, private communication. 


\section{CHAPTER IV}

EXPERIMENTAL PROGRESS IN STUDIES OF

FULLY STATE-RESOLVED DIFFERENTIAL CROSS SECTIONS

FOR THE D + $\mathrm{H}_{2}$ REACTION 


\begin{abstract}
The status of the crossed molecular beam experiment to measure rotationally stateresolved differential cross sections for the $\mathrm{D}+\mathrm{H}_{2}$ reaction is described. Tests results are reported related to the operation of the $D$ and $\mathrm{H}_{2}$ beams, the attenuation of the $D$ atom beam by the $\mathrm{H}_{2}$ beam, and sources of $(\mathrm{DH})$ background under full experimental conditions. Some preliminary reactive signal is reported also.
\end{abstract}




\section{Introduction}

In this chapter the progress will be reported that has been achieved to date in our experimental study of the fully state-resolved differential cross sections for the D $+\mathrm{H}_{2}$ reaction. Unfortunately a real angular distribution measurement has eluded us sofar, however, some good progress towards these measurements has been made, and it is with some confidence that one might anticipate the succesfull completion of these experiments in the very near future. The tests that have been carried out sofar relate to the operation of the $\mathrm{D}$ and $\mathrm{H}_{2}$ beams, the attenuation of the $\mathrm{D}$ atom beam by the $\mathrm{H}_{2}$ beam and sources of (DH) background under full experimental conditions. These tests will be discussed in this chapter. Some preliminary reactive signal which has been observed will be reported also.

Two different ion detectors were used in the tests described in this chapter. Most of the results were obtained with the position-sensitive microchannel plate detector described in the previous chapter. However, in the course of the experiments, this detector broke down several times, causing unfortunate delays in the experiment, and during some periods of time, when the position-sensitive detector was unavailable, a more conventional scintillation detector (Daly-detector) was used.

\subsection{Diagnostics for the $D$ atom beam}

In the experiments that have been carried out sofar, the photolysis source has been the 4th harmonic of a Nd:YAG laser at $266 \mathrm{~nm}$. Photolysis of DI at this wavelength produces $\mathrm{D}$ atoms with two velocities. As determined by Clear et. al. ${ }^{1)}, 74 \%$ of the DI molecules absorb through a perpendicular transition, producing a fast $D$ atom with a kinetic energy of $1.54 \mathrm{eV}$, and $26 \%$ of the DI molecules absorb through a parallel transition, producing a slower $\mathrm{D}$ atom with a kinetic energy of $0.61 \mathrm{eV}$. With reference to Figure 1 of the previous chapter, the fast $D$ atoms will preferentially recoil with a velocity vector in the reaction plane, if the photolysis laser is vertically polarized (i.e. out of the plane of Figure 1), and in this case a large number of fast $D$ 
atoms will enter the main chamber. The slower $D$ atoms recoil out of the reaction plane and only a small fraction of them will enter the main chamber.

Ry tuning the REMPI laser to the $3 \mathrm{~s}<-1$ s transition of the D atom, and varying the time delay between the photolysis and REMPI lasers, the time-of-flight spectrum of the D atoms entering the inain chamber can be recorded, as shown in Figure 1. The time-of-flight spectrum is dominated by a narrow peak due to the fast $D$ atoms, which reach the REMPI laser axis after about 2000 nanoseconds. The width of this peak is approximately 100 nanoseconds, and is primarily due to the size of the photolysis region and the angular divergence of the $D$ atom beam. It does not reflect the speed ratio of the beam, which cannot be measured very easily. At a time delay of about 3250 nanoseconds a small second peak appears, due to $\mathrm{D}$ atoms produced through the parallel transition. The observation of this peak is indicative of the occurrence of (in)elastic collisions in the photolysis region, as can be confirmed by comparing the top and bottom panel of Figure 1. In the uppar panel the DI pulsed valve was triggered $210 \mu$ seconds before the Q-switching of the photolysis laser, corresponding to a situation where th- photolysis laser interacted with the risiıg edge of the DI gas pulse. In the bottom panel the DI pulsed valve was triggered 40 useconds earlier, which means that the photolysis lasur encountered a more intense portion of the gas pulse, where the DI pressure was higher. Not only is the number of fast $D$ atoms produced higher in this case, but it can be seen that at the higher pressure the ratio between the peaks corresponding to fast and slow $\mathrm{D}$ atoms decreases, due to the increased possibility of velocity-changing collisions.

The time-of-flight spectra shown in Figure 1 were recorded using the positionsensitive microchannel plate detector, and are quite different from time-of-flight spectra which were recorded under similar conditions using the Daly-detector. In the latter experiments, after observation of the fast $\mathrm{D}$ atom peak around 2000 nanoseconds, a substantial tail was observed, with an intensity of as much as $25 \%$ of the fast $D$ atom peak. While these results were very similar to the results reported by Continetti ${ }^{2)}$, we now believe that these measurements may not be reliable due to saturation of the Daly-detector. In measuring these D atom time-of-flight spectra, an 


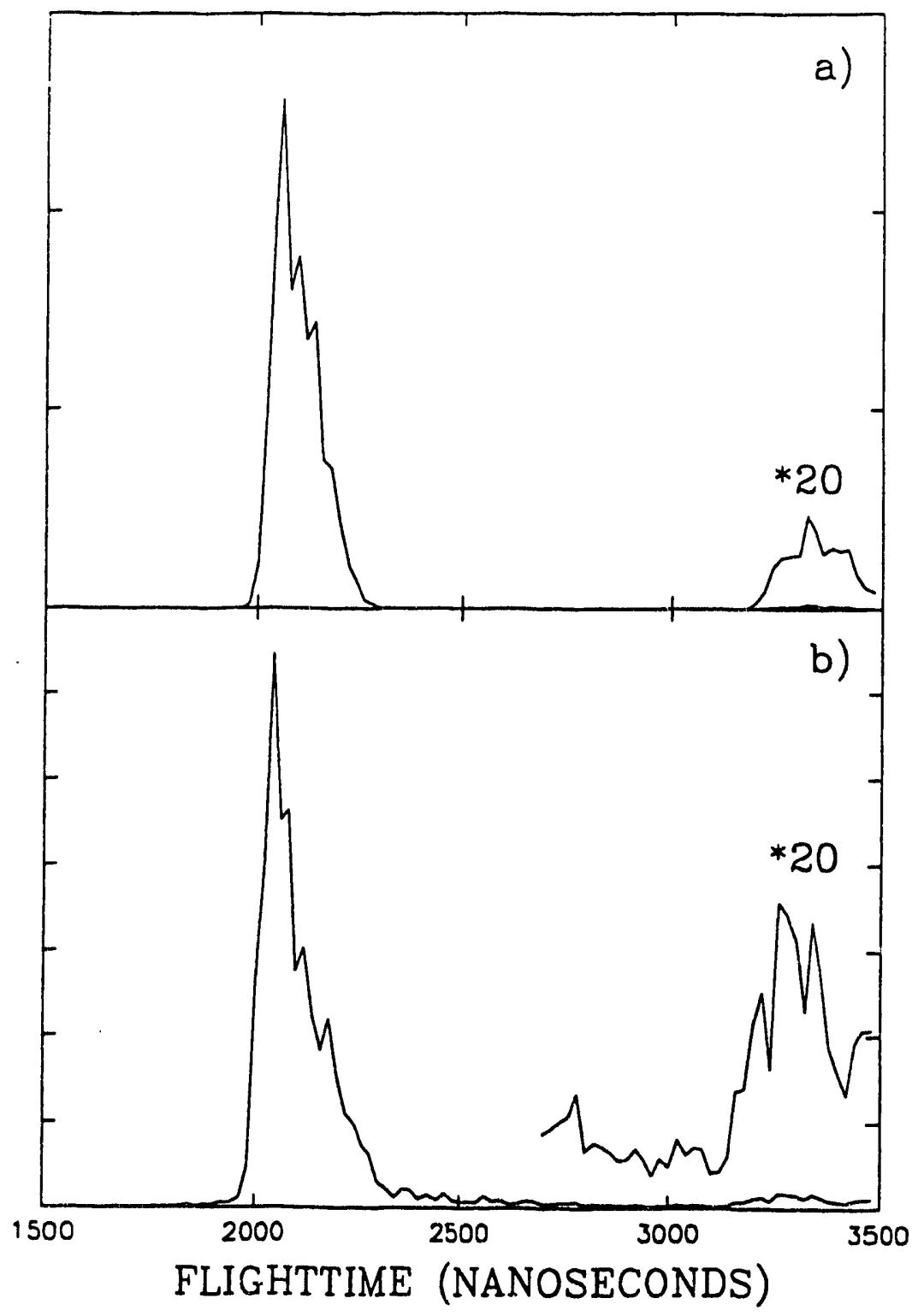

Figure 1 Time-of-flight spectra of photolytically produced D atoms with the DI pulsed valve trigger $210 \mu$ seconds (a), resp. $250 \mu$ seconds (b) prior to the Q-switching of the photolysis laser. The DI pressure in these measurements was approximately 200 Torr, and the $0.5 \mathrm{~mm}$ nozzle of the DI pulsed valve was mounted $1.0 \mathrm{~mm}$ below the reaction plane. 


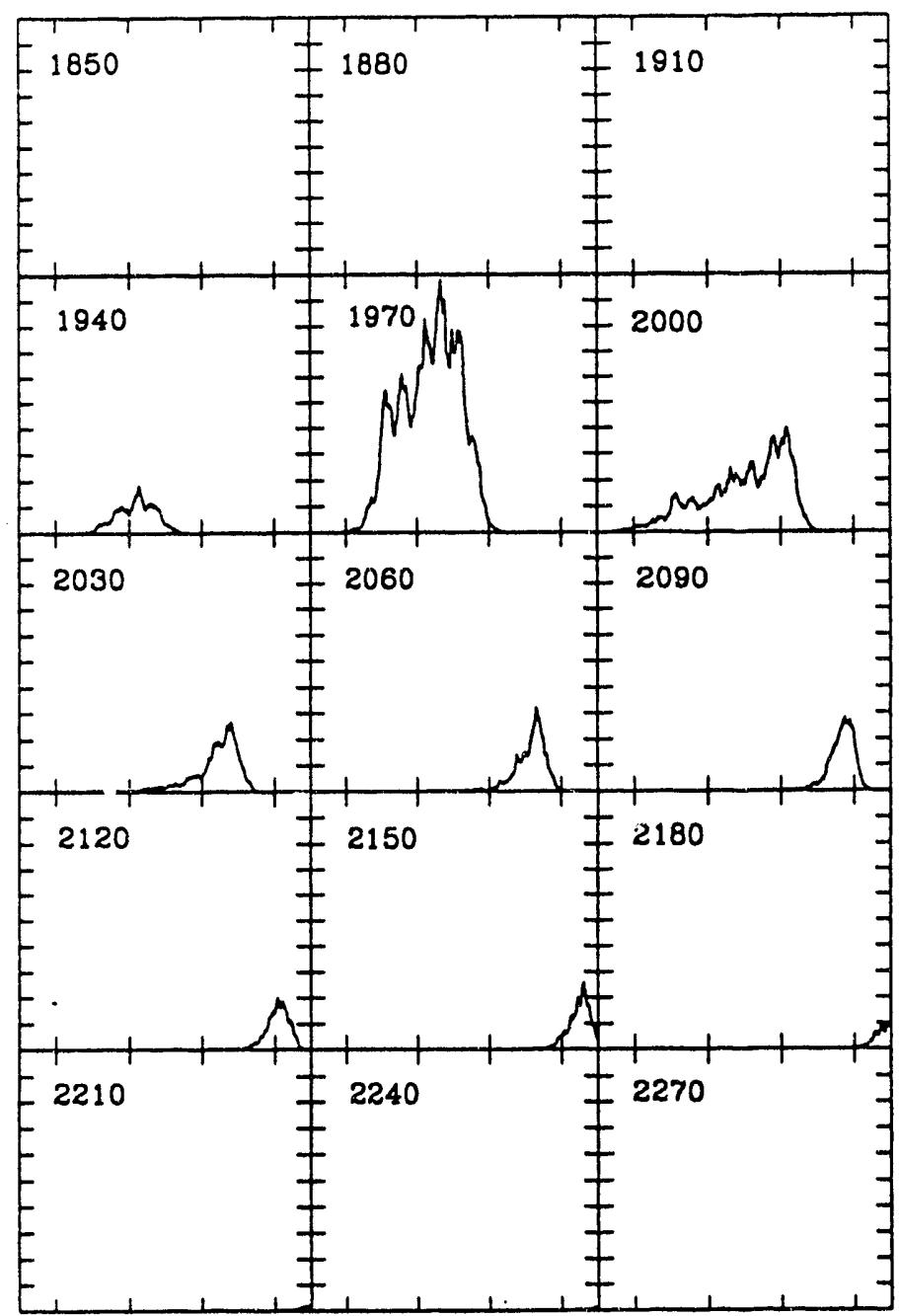

POSITION ALONG REMPI LASER AXIS

Figure 2 Distribution of $\mathrm{D}$ atoms along the REMPI laser axis as a function of the timedelay between the photolysis laser and the REMPI laser (in nanoseconds). The DI pressure in these measurements was approximately 120 Torr, and the $0.5 \mathrm{~mm}$ nozzle of the DI pulsed valve was mounted $1.0 \mathrm{~mm}$ below the reaction plane. 
estimated $10^{6}-10^{7}$ ions are formed per lasershot, which reach the detector in an approximately 20 nanosecond interval. For the Daly-detector this means that the photomultiplier is exposed to an instantaneous photon flux of as much as $10^{15}$ photons/second, which equals the photon flux of a small Helium Neon laser. While care was taken to ensure that the gain in the dynode chain of the photomultiplier was not saturated during these experiments, it seems reasonable to suspect that the photocathode might saturate at these high instantaneous photon fluxes, leading to unreliable results. Since the effective cathode area of the microchannel plate detector is significantly larger, saturation effects are expected to play a smaller role.

With the use of the position-sensitive detector it is possible to obtain some information about the spatial distribution of the $D$ atom beam. Figure 2 shows the distribution of $D$ atoms along the REMPI laser axis as a function of the time delay between the photolysis and REMPI lasers. With reference to Figure 1 of the previous chapter, it is observed that the initial D atom signal is due to $D$ atoms which travel almost perpendicular to the REMPI laser axis, and which therefore travel the shortest distance. As the time delay between the lasers is increased, $\mathrm{D}$ atoms travelling at the $17^{\circ}$ angle of the experimental design reach the laser axis and a strong increase in the signal is observed. Finally $D$ atoms at angles larger than $17^{\circ}$ are detected also. Figure 2 allows one to estimate the angular divergence of the $D$ atom beam at approximately $15^{\circ}$. In future experiments this angular divergence can be reduced by decreasing the size of the aperture separating the DI chamber from the main chamber, which was 0.7 $\mathrm{mm}$ in the current experiments, and by redusing the distance from the DI nozzle to the reaction plane. Figure 2 further illustrates that a significant portion of the broadening of the fast $D$ atom peak in Figure 1 is due to the angular divergence of the $D$ atom beam.

In Figure 3 an actual image is shown, as it appears on the video camera. In interpreting this image, it is important to realize that there is a time delay of about 500 nanoseconds between the ionization of the $\mathrm{D}$ atoms and the arrival of the $\mathrm{D}^{+}$ions at the microchannel plate. Consequently, depending on the velocities of the $D$ atoms parallel to the detector surface, the detected ions will show a displacement from the 


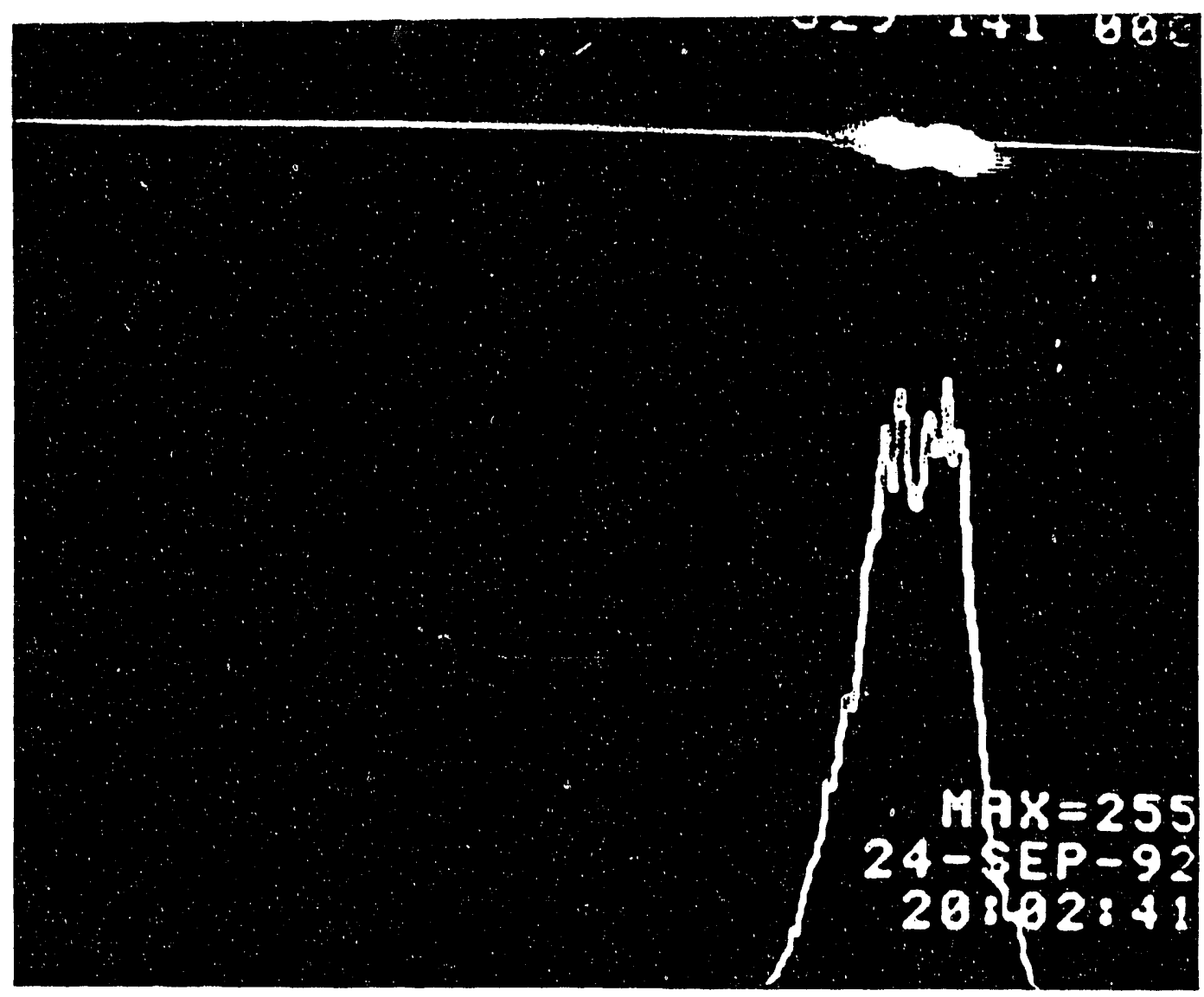

Figure 3 Typical image of the $\mathrm{D}$ atom beam recorded on the video camera, photographed at a time delay between the photolysis laser and the REMPI laser of 2070 nanoseconds. 
laser axis where they are produced. In this case, the $\mathrm{D}^{+}$ions retain the large in-plane velocity of the D atom beam, and, as expected from Figure 1 of the previous chapter, are detected in the top right portion of the detector.

As suggested by Figure 1, the timing between the triggering of the DI pulsed value and the photolysis laser plays a critical role in determining the characteristics of the $D$ atom beam. This point is further illustrated by Figure 4, which shows, for a number of DI backing pressures, the number of $D$ atoms detected at the time delay corresponding to the fast $\mathrm{D}$ atom peak, as a function of the delay between the triggering of the DI pulsed valve and the Q-switching of the photolysis laser. At the lowest pressures, the appearance of the fast $D$ atoms closely follows the DI gas pulse, indicating that most of the $\mathrm{D}$ atoms escape the photolysis region without secondary collisions. As the DI backing pressure is increased to approximately 150 Torr however, a dip appears at the center of the DI gas pulse, where the DI pressure is highest. In this regime a substantial fraction of the $D$ atoms produced undergo velocity changing collisions. This dip becomes even more pronounced as the pressure is increased further. At the higher pressures a loss of $D$ atom production due to the formation of DI clusters is expected also.

As the DI backing pressure is increased, there appears a second peak at long time delays between the DI gas pulse and the photolysis and REMPI lasers, which only depends on the presence of the REMPI laser. This peak is due to the fact that the operation of the DI pulsed valve leads to a modest build-up of the DI partial pressure in the main chamber. The $205 \mathrm{~nm}$ REMPI laser, which ionizes the D atoms, efficiently dissociates DI molecules, resulting in the detection of additional signal. The REMPI laser induced signal provides a convenient method to monitor the isotopic purity of the DI sample. Typically, after the passivation of the DI gas lines and the DI pulsed valve is completed, the isotopic purity of the DI gas is estimated to be at least $98 \%$. In appendix $\mathrm{A}$ a further analysis of the REMPI laser induced signal is presented.

An additional diagnostic for the timing dependence between the DI pulsed valve and the photolysis laser is presented in Figure 5. Similar to Figure 2, the distribution of $\mathrm{D}$ atoms along the laser axis is shown in this figure, as a function of 


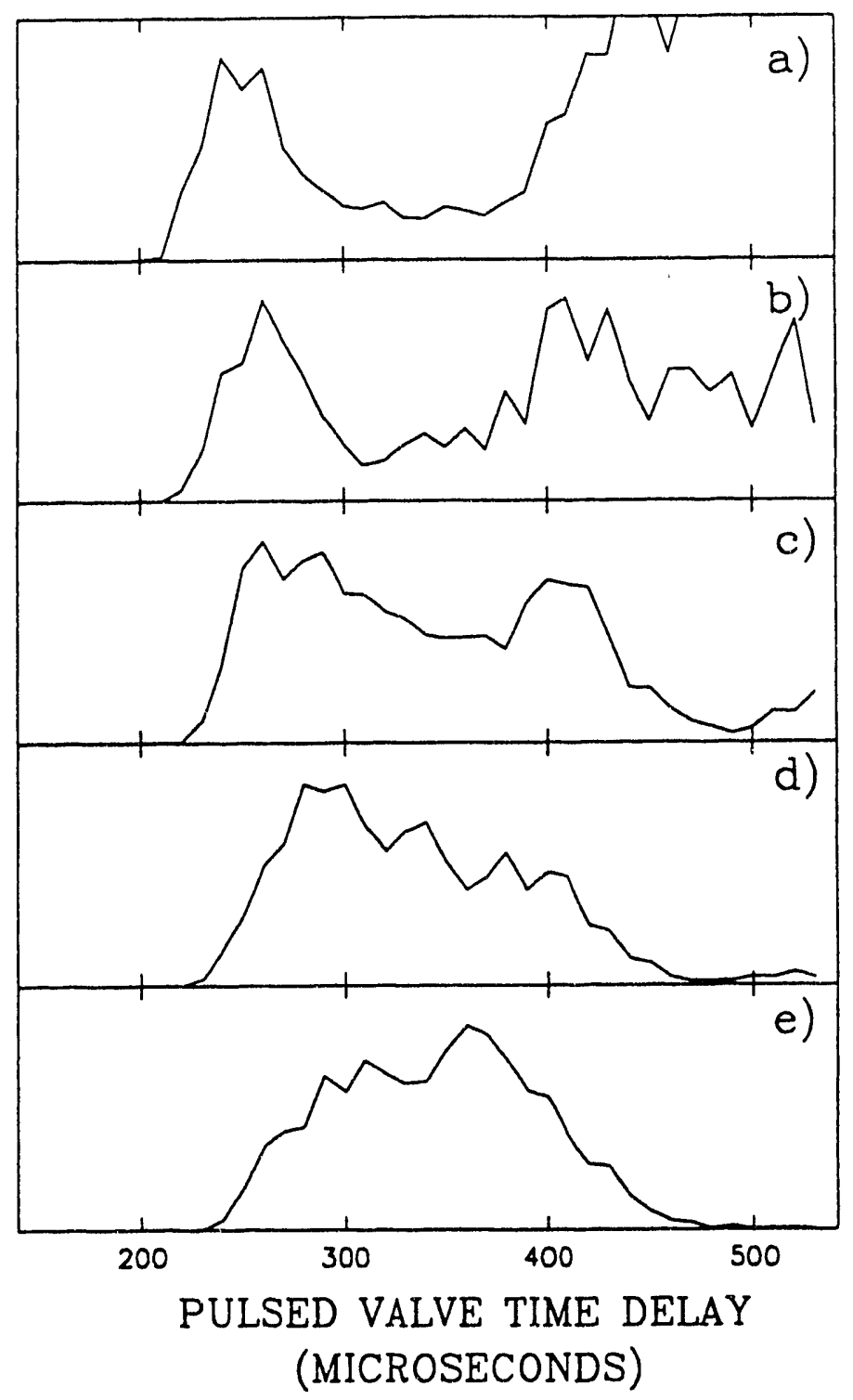

Figure 4 Dependence of the number of $D$ atoms detected on the fast $D$ atom peak as a function of the delay between the trigger pulse for the DI pulsed valve and the Q-switching of the photolysis laser. The time delay between the photolysis and REMPI lasers was fixed at 2070 nanoseconds. The experiments were performed with the $0.5 \mathrm{~mm}$ nozzle of the DI pulsed valve mounted $1.0 \mathrm{~mm}$ below the reaction plane. The DI backing pressures were a) 470 Torr, b) 260 Torr, c) 165 Torr, d) 125 Torr and e) 90 Torr. 


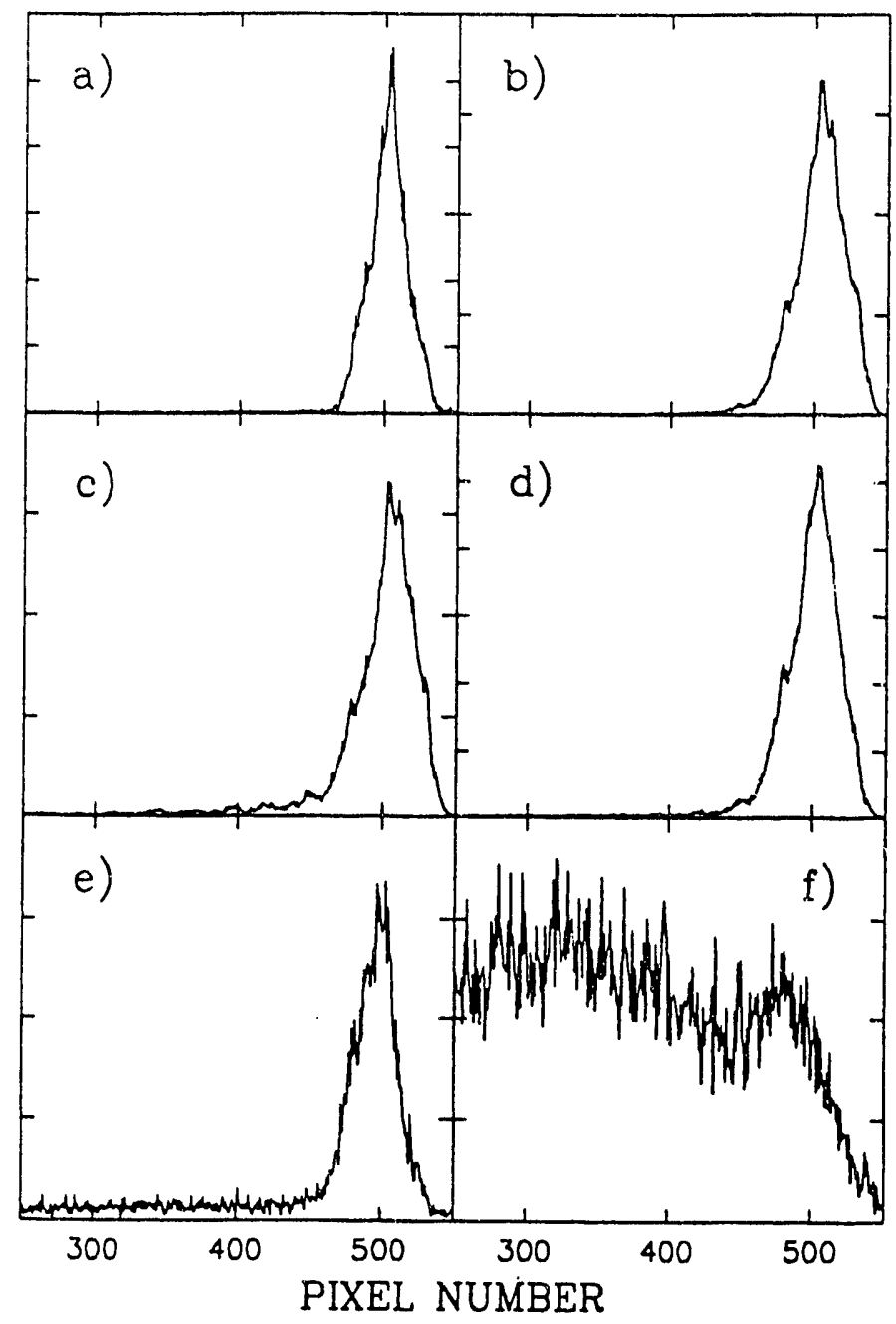

Figure 5 Distribution of $\mathrm{D}$ atoms along the REMPI laser axis, as a function of the time delay between the triggering of the DI pulsed valve and the Qswitching of the photolysis laser. The conditions are those of Figure 4c. The time delays between the triggering of the DI pulsed valve and the Q-switching of the photolysis laser were a) $230 \mu$ seconds, b) 290 $\mu$ seconds, c) $350 \mu$ seconds, d) $410 \mu$ sec unds, e) $470 \mu$ seconds and f) $530 \mu$ seconds. 
the time delay between the triggering of the DI pulsed valve and the Q-switching of the photolysis laser, for the measurement with the DI backing pressure at 165 Torr (Figure 4c). In Figure 5 the time delay between the photolysis and REMPI laser is fixed at 2070 nanoseconds. It is observed that the occurrence of the dip in Figure 4c corresponds to a regime where the D atom spatial profile shows collisional broadening, which at this time delay between the photolysis and REMPI lasers narticularly shows up on the left side of the main $D$ atom peak. At the longer time delays shown in the two bottom panels, the influence of the REMPI laser induced D atom production is clearly seen, which creates a uniform background. Based on the results shown in Figures 4 and 5, the DI pulsed valve is typically operated at a backing pressure of approximately 100 Torr and a time delay between the pulsed valve trigger and the photolysis laser Q-switch of about 250 useconds.

Finally, brief mention should me made of a study of the dependence of the D atom production on th. power of the $266 \mathrm{~nm}$ photolysis laser. Initial measurements, using the Daly-d'tector, showed a leveling-off of the $D$ atom beam intensity at a photolysis laser power of approximately $25 \mathrm{~mJ} /$ pulse. However, in light of the aforementioned concems about saturation of the Daly-detector, it may be necessary to repeat these experiments with the position-sensitive ion detector.

\subsection{Diagnostics for the $\mathrm{H}_{2}$ beam}

Initial tests with the pulsed valve used to generate the $\mathrm{H}_{2}$ beam were performed on one of the universal crossed molecular beam machines in the Lee-group. These tests showed that the skimmed $\mathrm{H}_{2}$ beam moved with a very narrow velocity distribution, where all of the initial rotational energy had been converted to translational energy. At typical backing pressures, these measurements put an instrument-limited lower limit on the speed ratio of the $\mathrm{H}_{2}$ beam of about 12 .

Further tests on the $\mathrm{H}_{2}$ beam characteristics were done in situ by placing a fast ionization gauge according to the design of Gentry and Giese ${ }^{3)}$ in the main chamber, approximately $4 \mathrm{~cm}$ downstream from the crossing point with the $\mathrm{D}$ atom beam. 
Results of these tests are shown in Figures 6 and 7.

In Figure 6 the dependence of the intensity of the $\mathrm{H}_{2}$ gas pulse on the voltage applied to the piezocrystal is shown. In the homebuilt pulse valve used to create the $\mathrm{H}_{2}$ gas pulse, a $150 \mu$ seconds long voltage pulse is applied to a piezocrystal, supporting an O-ring seal covering the nozzle, which had a diameter of $0.75 \mathrm{~mm}$ in these experiments. With the first part of the voltage pulse the compression on the $\mathrm{O}$ ring seal is removed and subsequently the seal is pulled back in order to achieve nozzle-limited flow. Figure 6 shows how, with the valve adjusted for a threshold around 250 Volts, the valve provides near nozzle-limited flow when supplied with voltage pulses above 600 Volts, generating an approximately $300 \mu$ seconds long pulsed $\mathrm{H}_{2}$ beam. Above 600 Volts, the force exerted on the piezocrystal during the reclosing of the seal induces a second bounce appearing about $500 \mu$ seconds after the initial gas pulse, in agreement with the fact that the piezocrystals used (Model P 910.160, Physik Instrumente $\mathrm{GmbH}$ ) have an eigenfrequency of approximately 2.5 kHz.

In Figure 7 the dependence of the pulsed $\mathrm{H}_{2}$ beam on the $\mathrm{H}_{2}$ backing pressure is shown. The peak intensity is nearly linear with the $\mathrm{H}_{2}$ backing pressure, suggesting that the pulsed $\mathrm{H}_{2}$ jet is skimmed without substantial attenuation. The results shown in Figures 6 and 7 were obtained using a $15 \mathrm{~mm}$ nozzle-skimmer distance and a $0.75 \mathrm{~mm}$ diameter $8 \mathrm{~mm}$ long electro-formed skimmer (Beam Dynamics). As shown in Figure 8 , the successfull generation of an intense $\mathrm{H}_{2}$ beam cannot be taken for granted. Along with the results obtained for the $8 \mathrm{~mm}$ long skimmer, this figure shows results obtained with a $0.75 \mathrm{~mm}$ diameter $15 \mathrm{~mm}$ long stainless steel skimmer. The major reduction in the $\mathrm{H}_{2}$ beam intensity observed in this case strongly suggests that with this skimmer the $\mathrm{H}_{2}$ beam intensity was attenuated by collisions inside the narrower skimmer cone and/or increased collisions with $\mathrm{H}_{2}$ molecules bouncing off the skimmer and the walls of the $\mathrm{H}_{2}$ source chamber. The reduction in the $\mathrm{H}_{2}$ beam intensity can not be explained by the fact that the nozzle was slightly farther from the fast ionization gauge in this case. Even more dramatic are the results that were obtained when a second skimmer was placed between the afore-mentioned $8 \mathrm{~mm}$ long electro- 


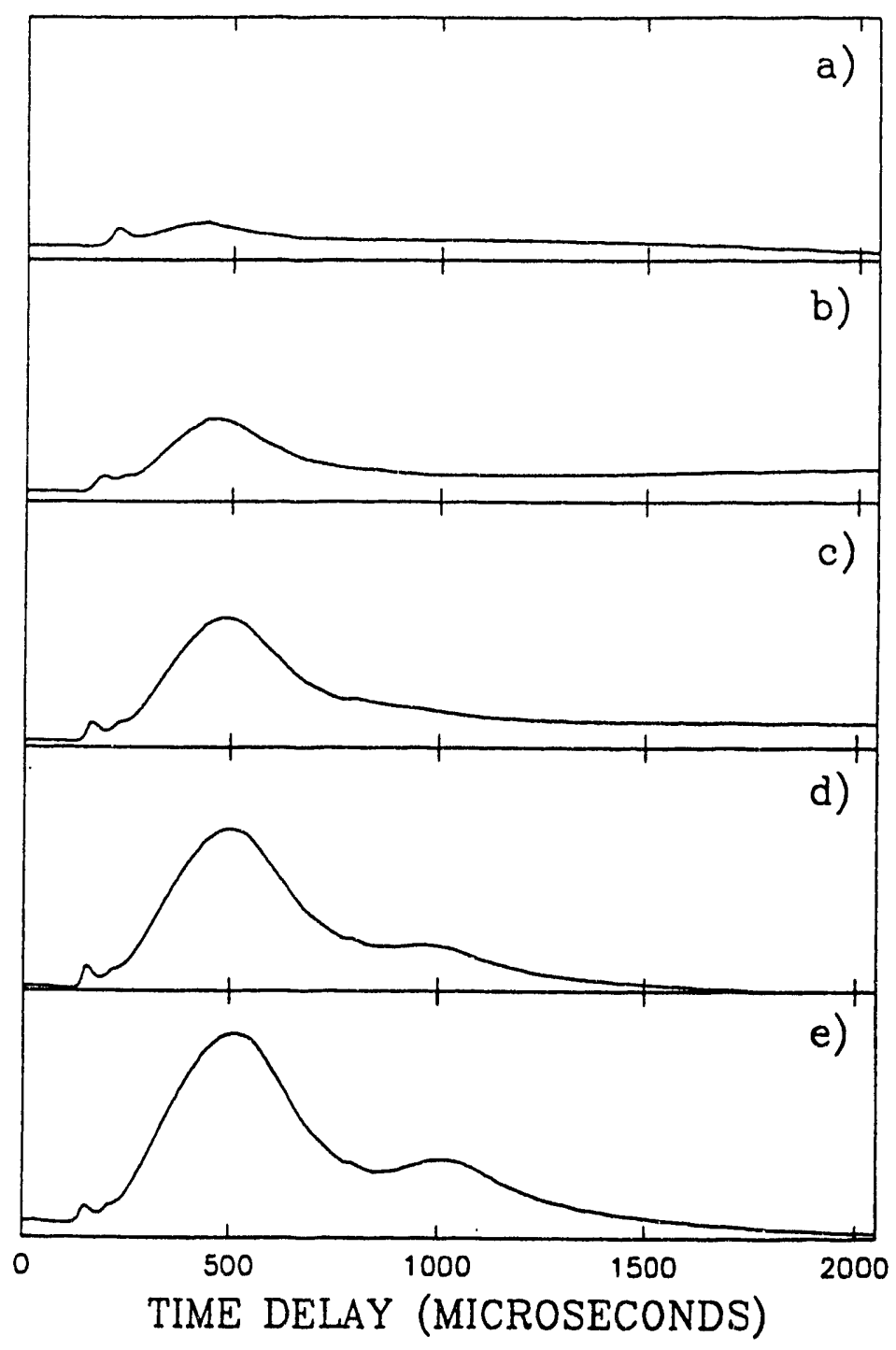

Figure 6 Dependence of the intensity of the pulsed $\mathrm{H}_{2}$ beam on the voltage applied to the piezocrystal of the $\mathrm{H}_{2}$ pulsed valve, measured using a fast ionization gauge. The $\mathrm{H}_{2}$ backing pressure was 25 psig and the $\mathrm{H}_{2}$ nozzle diameter was $0.75 \mathrm{~mm}$. The $\mathrm{H}_{2}$ jet was skimmed $15 \mathrm{~mm}$ from the nozzle with an $8 \mathrm{~mm}$ long, $0.75 \mathrm{~mm}$ diameter electro-formed skimmer. The $150 \mu$ second long voltage pulses were a) 300 Volts, b) 400 Volts, c) 500 Volts, d) 600 Volts and e) 700 Volts high: 


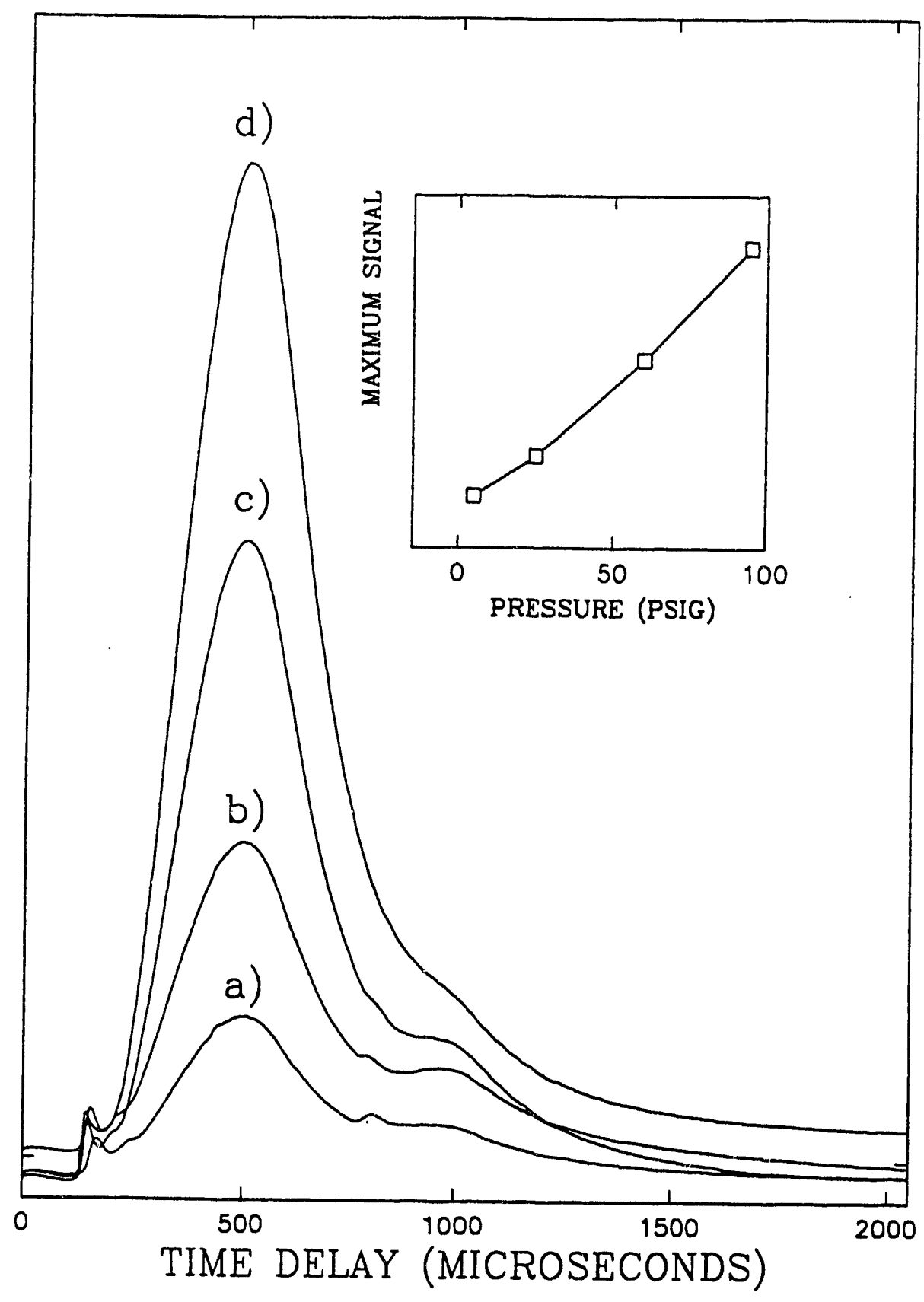

Figure 7 Dependence of the intensity of the pulsed $\mathrm{H}_{2}$ beam on the backing pressure. The experimental conditions were those of Figure 6. The $\mathrm{H}_{2}$ backing pressures used were a) 5 psig, b) 25 psig, c) 60 psig and d) 100 psig, respectively. 


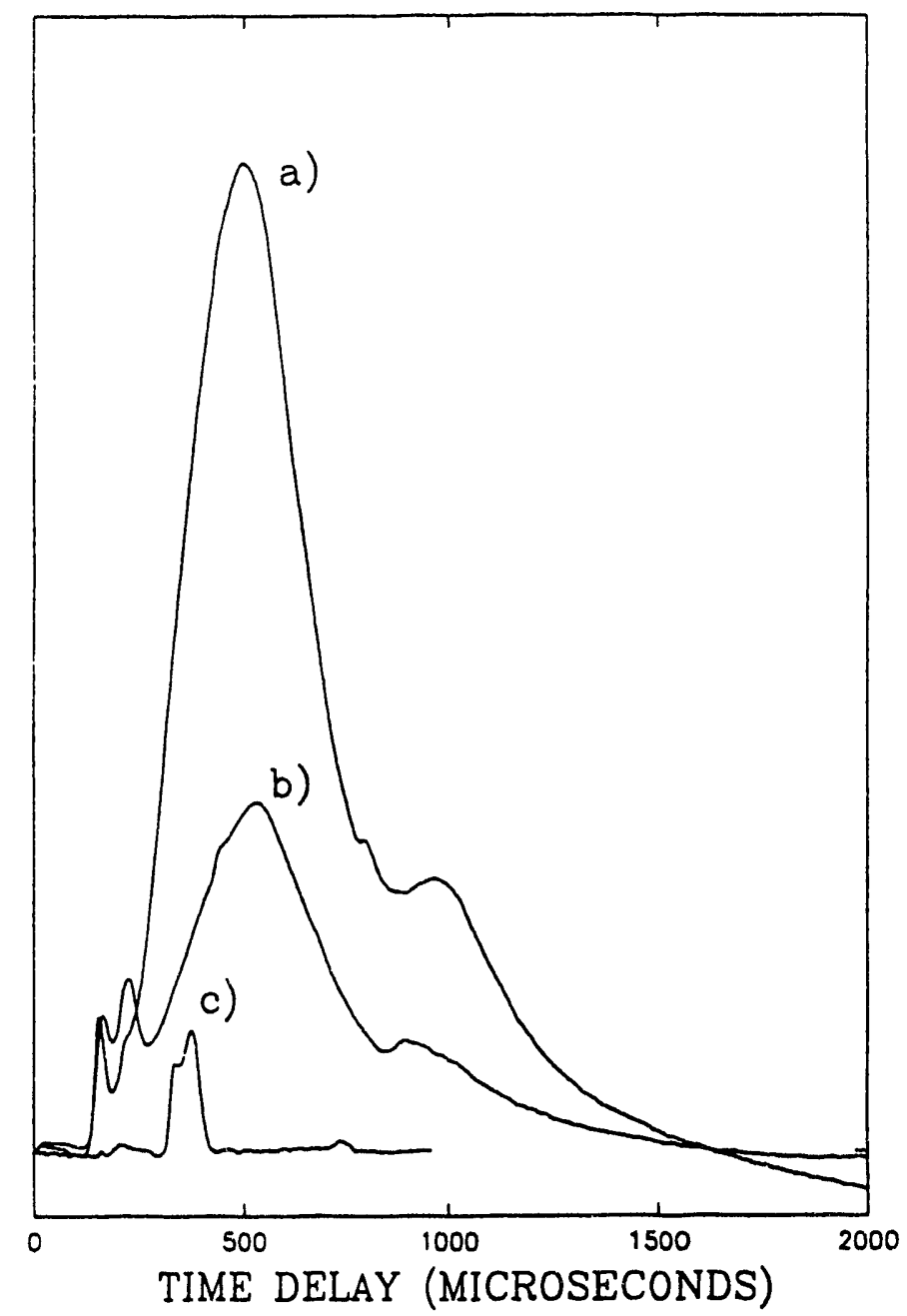

Figure 8 Dependence of the intensity of the pulsed $\mathrm{H}_{2}$ beam on the nozzleskimmer configuration. Trace a) was recorded using an $8 \mathrm{~mm}$ long 0.75 $\mathrm{mm}$ diameter electro-formed skimmer, with a $15 \mathrm{~mm}$ nozzle-skimmer distance, trace b) was recorded with a $15 \mathrm{~mm}$ long $0.75 \mathrm{~mm}$ diameter stainless steel skimmer and a $15 \mathrm{~mm}$ nozzle-skimmer distance, and trace c) was recorded with a doubly skimmed configuration, using the $8 \mathrm{~mm}$ long $0.75 \mathrm{~mm}$ electro-formed skimmer, mounted with a nozzle-skimmer distance of $15 \mathrm{~mm}$, followed $20 \mathrm{~mm}$ downstream by a second $5 \mathrm{~mm}$ long $1.25 \mathrm{~mm}$ diameter skimmer. The backing pressure in these measurements was 25 psig and the $\mathrm{H}_{2}$ pulsed valve was operated with a $150 \mu$ seconds long, 600 Volt pulse. 
formed skimmer, suspended on a separate differential cone, and the main chamber.

This geometry was used in an attempt to improve the collimation of the $\mathrm{H}_{2}$ beam, but the accumulation of $\mathrm{H}_{2}$ gas between the secondary cone and the wall separating the $\mathrm{H}_{2}$ chamber from the main chamber, led to a major attenuation of the beam intensity, completely extinguishing the $\mathrm{H}_{2}$ beam except for the rising and falling edges of the $\mathrm{H}_{2}$ gas pulse, where the $\mathrm{H}_{2}$ pressure is relatively low.

\subsection{Attenuation of the $D$ atom beam}

In order to determine the appropriate conditions for the operation of the $\mathrm{H}_{2}$ beam, where a limited number of the $\mathrm{D}$ atoms (and the $\mathrm{DH}$ molecules formed) collide with $\mathrm{H}_{2}$ molecules, the time delay between the $\mathrm{H}_{2}$ pulsed valve and the Q-switching of the photolysis and REMPI lasers was varied, while monitoring the arrival of fast $D$ atoms at the REMPI laser axis. In these experiments, $\mathrm{H}_{2}$ at a pressure of 95 psig was expanded through a $0.75 \mathrm{~mm}$ nozzle, located $15 \mathrm{~mm}$ from an $8 \mathrm{~mm}$ long $0.75 \mathrm{~mm}$ diameter electro-formed skimmer. Figure 9 shows how the intensity of the $D$ atom beam shows a dip corresponding to the temporal profile of the pulsed $\mathrm{H}_{2}$ beam, and that, if the $\mathrm{H}_{2}$ pulsed valve is triggered 3-400 $\mu$ seconds prior to the generation of the $D$ atom beam, the $\mathrm{H}_{2}$ pressure at the crossing point is high enough to attenuate the $D$ atom beam intensity by as much as $70 \%$. As discussed in the previous chapter, Continetti ${ }^{2}$ carried out his experiments under a condition where about $1.8 \%$ of the DH molecules formed undergo a.hard-sphere collision with an $\mathrm{H}_{2}$ molecule while leaving the crossing region. The result of Figure 9 suggests that in the current experiment, one can afford to pull back the $\mathrm{H}_{2}$ pulsed valve and reduce the $\mathrm{H}_{2}$ nozzle and skimmer sizes significantly, in order to improve the collimation of the $\mathrm{H}_{2}$ beam. 


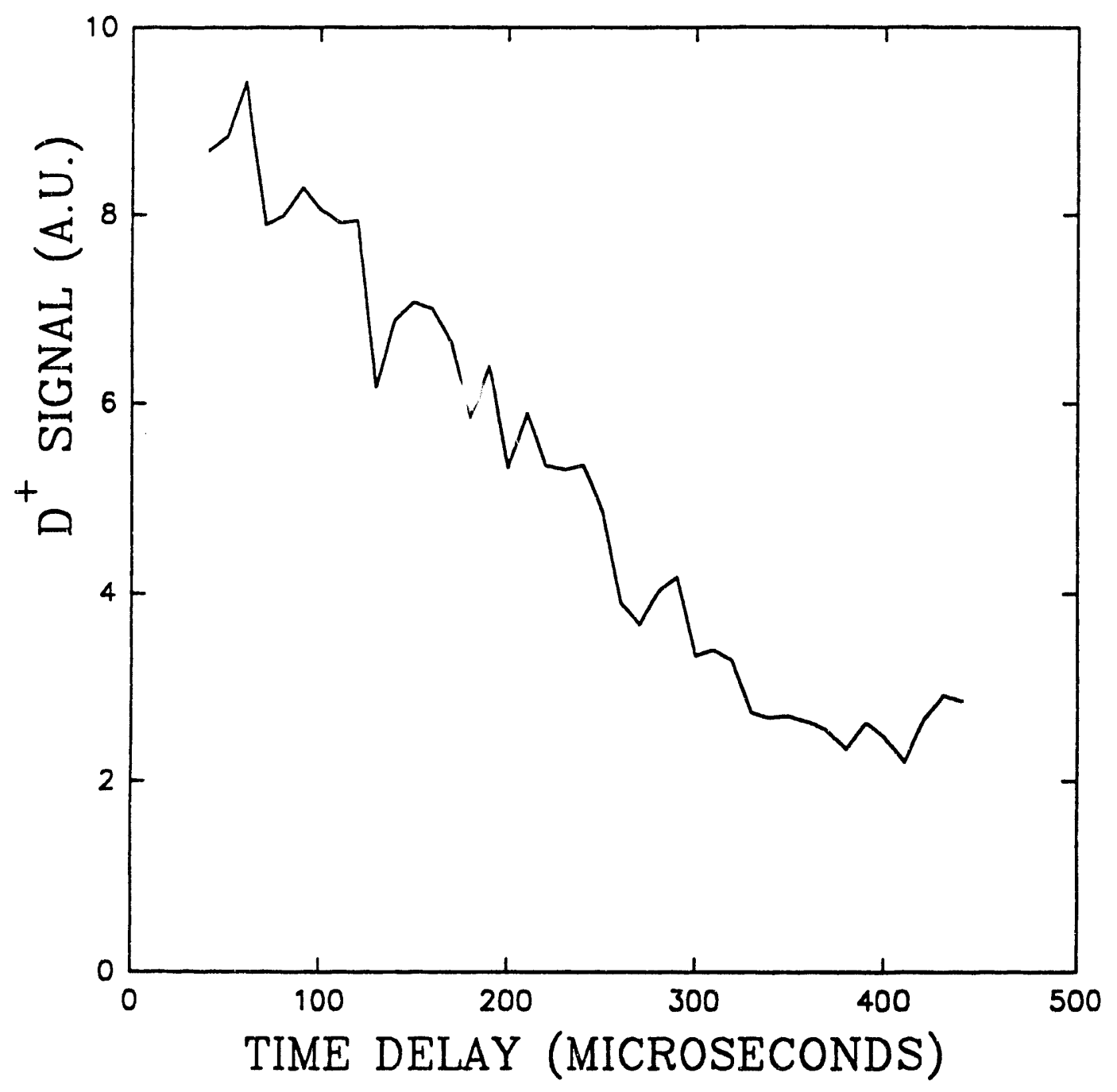

Figure 9 Attenuation of the $D$ atom beam by the $\mathrm{H}_{2}$ beam. The $\mathrm{H}_{2}$ beam was operated by expanding 95 psig of $\mathrm{H}_{2}$ through a $0.75 \mathrm{~mm}$ nozzle, located $15 \mathrm{~mm}$ from an $8 \mathrm{~mm}$ long $0.75 \mathrm{~mm}$ diameter electro-formed skimmer. 


\subsection{Molecular hydrogen in the $\mathrm{D}$ atom beam}

As discussed in the experimental description of Chapter 3, care was taken to design an experiment in which no signal would be recorded, unless DH molecules in a selected ro-vibrational quantum state would be present in the REMPI laser detection volume. In this section some attention will be devoted to a discussion of experimentally observable DH molecules from other sources than reactions with the $\mathrm{H}_{2}$ beam.

The $\mathrm{D}$ atom beam is expected to contain limited amounts of DH. The DI sample used contains impurity HI molecules and, as discussed in the previous chapter, the pressure in the photolysis region is such that about $35 \%$ of the hydrogen atoms formed undergo a collision before leaving the photolysis region, including both $\mathrm{D}+\mathrm{HI}$ and $\mathrm{H}+\mathrm{DI}$ reactive collisions. Signal from these reactions could readily be observed in the crossed molecular beam experiments of Continetti ${ }^{2}$, whose DH time-of-flight spectra showed $\mathrm{DH}$ molecules from both $\mathrm{D}+\mathrm{H}_{2}$ reactions and the reactions discussed above.

In our first attempt to observe reactive signal from the $\mathrm{D}+\mathrm{H}_{2}$ reaction, the detection laser was tuned to $\operatorname{DH}(j=7)$, a state which accounts for about $10 \%$ of the products formed. In addition to signal which depended on both molecular beams and both the photolysis and the REMPI lasers, a rather surprising amount of DH was detected which was part of the $D$ atom beam. For example, with the DI pulsed valve mounted $1 \mathrm{~mm}$ below the reaction plane and operated at a backing pressure of approximately 200 Torr, $\mathrm{DH}^{+}$signals as large as 30 ions/lasershot were detected. These DH molecules move at relatively large velocities, with the peak in the velocity distribution appearing at about $80 \%$ of the $D$ atom beam velocity.

In order to clarify the origin of the formation of this DH signal, a comparison was made with the amount of $D_{2}$ in the $D$ atom beam. Based on a comparison of the REMPI laser induced atomic hydrogen signal resulting from the dissociation of $\mathrm{HI}$ and DI, the isotopic purity of the DI sample is typically estimated to be at least $98 \%$, leading to the prediction that the amount of $D_{2}$ produced should exceed the amount of DH by about a factor of 25 . The signal detected for $\mathrm{DH}(\mathrm{j}=7)$ was compared to that for 
$D_{2}(j=9)$, a state which has a very comparable rotational energy, and which is detected at an almost identical REMPI laser frequency, avoiding possible complications due to the strong wavelength dependence of the REMPI laser power.

Under the experimental conditions described above, the signals obtained for $\mathrm{DH}(\mathrm{j}=7)$ and $\mathrm{D}_{2}(\mathrm{j}=9)$ were found to be almost identical. In addition to this rather surprising result, it was found that the dependence of the $\mathrm{DH}(\mathrm{j}=7)$ signal on the DI backing pressure was radically different from that of the $D_{2}(j=9)$ signal. When the DI backing pressure was reduced from 200 Torr to 50 Torr, the $D_{2}$ signal dropped by almost two orders of magnitude, whereas the DH signal dropped by only a factor of 7 . These observations led to the conclusion that the $\mathrm{DH}$ and $\mathrm{D}_{2}$ signals were produced by two different mechanisms. Whereas the $D_{2}$ is probably exclusively due to gas phase $D$ $+\mathrm{DI}$ collisions, the $\mathrm{DH}$ signal is dominated by $\mathrm{H}$ atom abstraction reactions on surfaces, such as the nozzle plate and the aperture separating the DI chamber from the main chamber. Further evidence for this assertion can be provided in two ways. First of all, increasing the distance from the nozzle plate to the reaction plane from $1 \mathrm{~mm}$ to $2 \mathrm{~mm}$, while maintaining the same pressure in the photolysis volume, reduced the $D H(j=7): D_{2}(j=9)$ ratio by a factor of 15 . And further credibility for a surface mechanism is provided by the fact that the $\mathrm{DH}$ in the $\mathrm{D}$ atom beam is not the only background DH signal that is observed. Figure 10 shows two images of the DH background signal, recorded at different time delays between the Q-switches of the photolysis and REMPI lasers. In Figure 10a the time delay between the photolysis and REMPI lasers is $2.5 \mu$ seconds, and the image shows DH molecules moving along with the $D$ atom beam at large velocities, causing a large displacement between the position where the DH molecules are ionized and the position on the position-sensitive detector where the $\mathrm{DH}^{+}$ions are detected. In Figure $10 \mathrm{~b}$ the time delay between the photolysis and REMPI lasers is $6 \mu$ seconds, and now, in addition to DH molecules moving along the $\mathrm{D}$ atom beam direction, where the REMPI laser time delay now selects somewhat slower $\mathrm{DH}$ molecules, there appears an additional $\mathrm{DH}^{+}$signal below the REMPI laser axis, indicating a $D H$ velocity opposite to the $D$ atom beam velocity. This signal is due to $D$ atoms on the vertical extremes of the $D$ atom beam, which, approximately 3 
$\mu$ seconds after the photolysis, collide with the central extraction electrodes while exiting the detector region, and react to form $\mathrm{DH}$ molecules which bounce back into the detector and are detected a little later.

A number of different approaches were taken to reduce the $\mathrm{DH}$ background signal. As mentioned before, pulling back the nozzle proved to be an efficienct method to reduce the DH signal, however this method is ultimately undesirable, since the divergence of the $\mathrm{D}$ atom beam increases if the photolysis volume is extended along the photolysis laser axis. The DI nozzle plate was redesigned, and a $5 \mathrm{~mm}$ long knifeedge capillary tube was attached to the nozzle plate, in order to minimize the effective surface area. Surprisingly, no obvious improvement was found. Therefore, using infrared heating lamps the DI source chamber and the main chamber were baked for about a week, leading to an estimated reduction of the $\mathrm{DH}$ background in the $\mathrm{D}$ atom beam of up to one order of magnitude, i.e. down to a level where gas phase DH production could in principle account for most of the DH observed.

One complication in comparing the $\mathrm{DH}$ background under the various conditions that have been tried, is that, while the Doppler-free $(2+1)$ REMPI detection is invariably very efficient, the actual detection efficiency from one experiment to the next is not very good, and may easily vary by as much as a factor of 2 . This, coupled with the problem of repeatedly reproducing source conditions with the same isotopic purity for the DI sample and the same photolysis region pressure, makes very precise quantitative comparisons hard. Therefore, while at this moment our preliminary conclusion would be that with extensive baking the $\mathrm{DH}$ content in the $\mathrm{D}$ atom beam can be reduced, the DH background needs to be monitored very closely in the future and some of the observations described above may need to be reconsidered. 


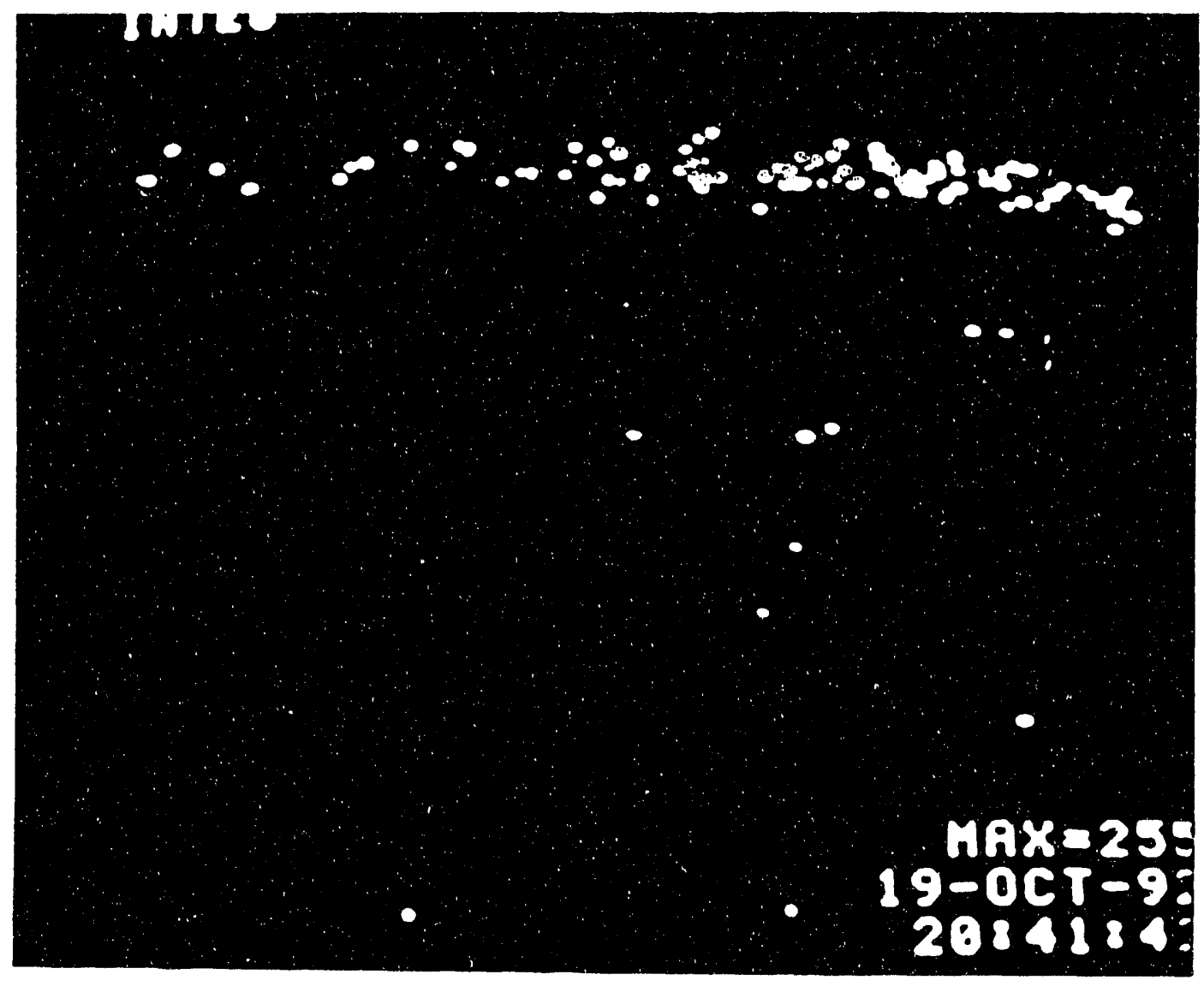

Figure 10a

Figure 10 Images of the DH background signal, observed when only the D atom beam is operated. The time delay between the photolysis laser and the REMPI laser was a) $2.5 \mu$ seconds and b) $6 \mu$ seconds. The DI pressure in these measurements was approximately 100 Torr, and the $0.5 \mathrm{~mm}$ nozzle of the DI pulsed valve was mounted $1.0 \mathrm{~mm}$ below the reaction plane. 


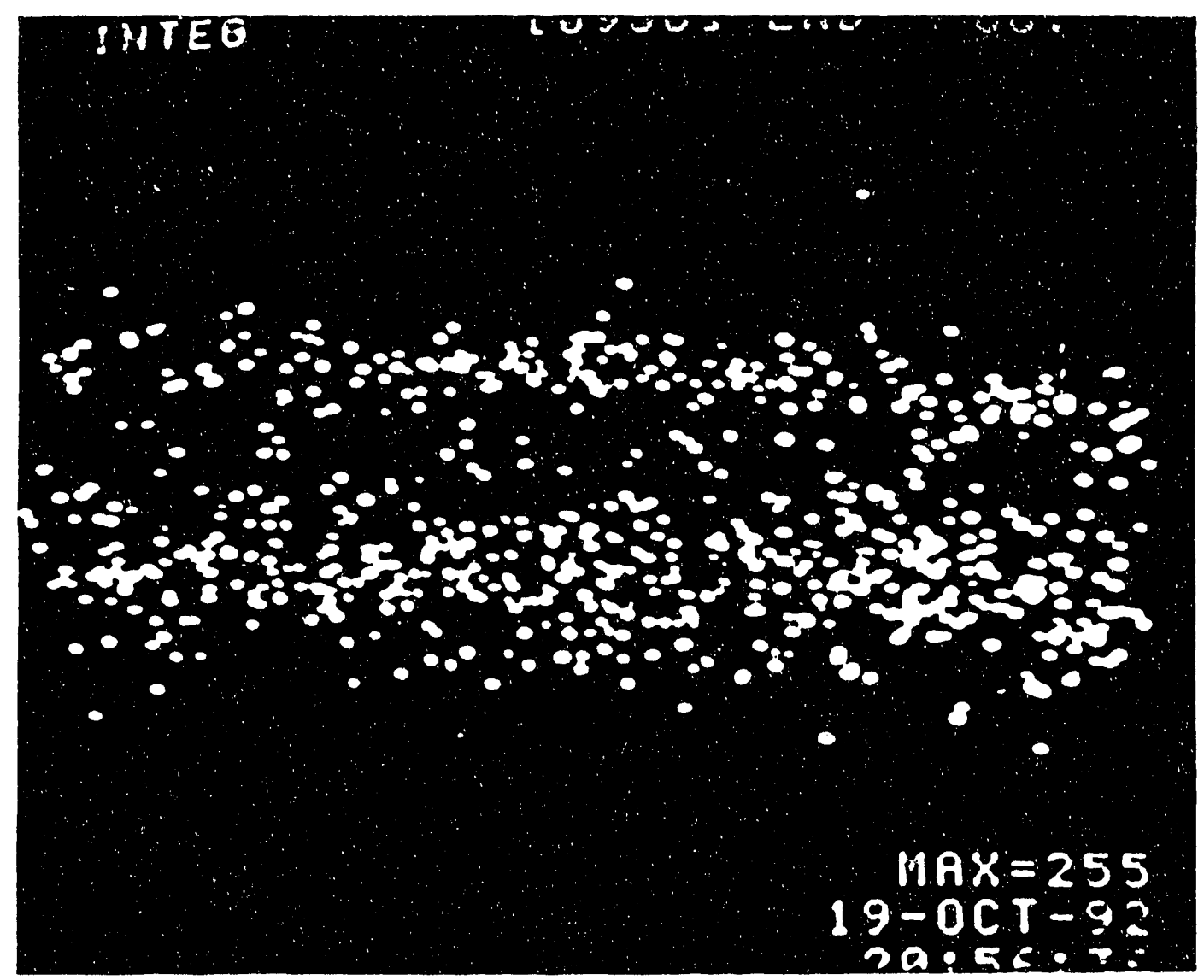

Figure $10 b$ 
2.5 The first observation of possible signal from $\mathrm{D}+\mathrm{H}_{2}$ and outlook for the future

In Figure 11 the first results are shown of an experiment where DH molecules were detected, whose observation depended on all four of the required components in this experiment, namely, the presence of the photolysis laser, Doppler-free detection by the counterpropagating REMPI lasers, operation of the DI pulsed valve and operation of the $\mathrm{H}_{2}$ pulsed valve. With the REMPI laser tuned to detection of $\mathrm{DH}(\mathrm{j}=7)$, a signal of about one ion per lasershot was recorded for time delays between the photolysis and REMPI lasers which corresponded to the detection of forward scattered DH product. While the DH product from the reaction is expected to be predominantly backscattered, it turned out that it was the forward scattered product which could most easily be observed. This is caused by the fact that the forward scattered product appears at time delays where the $\mathrm{DH}$ molecules formed in the reaction with $\mathrm{H}_{2}$, remain somewhat spatially separated on the position-sensitive detector, from the background DH molecules from the sources discussed in the previous section, which in this experiment exhibited countrates as high as 30 counts per lasershot. As seen in Figure $10 \mathrm{~b}$, at longer time delays between the photolysis and REMPI lasers, the DH signal from the afore-mentioned surface reactions increasingly obscures any $\mathrm{H}_{2}$ beamdependent signal, even though Figure 11 suggests that the $\mathrm{H}_{2}$ beam-dependent signal might still continue to increase at these time delays.

While Figure 11 shows a promise that the observation of $\mathrm{DH}$ angular distributions may be within reach shortly, the results of Figure 11 itself should probably not be over-emphasized. Sofar, the $\mathrm{H}_{2}$ beam-dependent signal has not displayed the characteristic 'double peak' structure that is anticipated for the data in this experiment (see Figure 2 of the previous chapter), and some important tests, such as the replacement of the $\mathrm{H}_{2}$ beam by, for example, a Helium beam, have not yet been carried out. Thus, it cannot be completely ruled out yet that the observed DH might be due to (in)elastic scattering of DH molecules in the $\mathrm{D}$ atom beam, although it would seem the $\mathrm{H}_{2}$ beam-dependent $\mathrm{DH}$ signal is too large to be explained by this mechanism. 


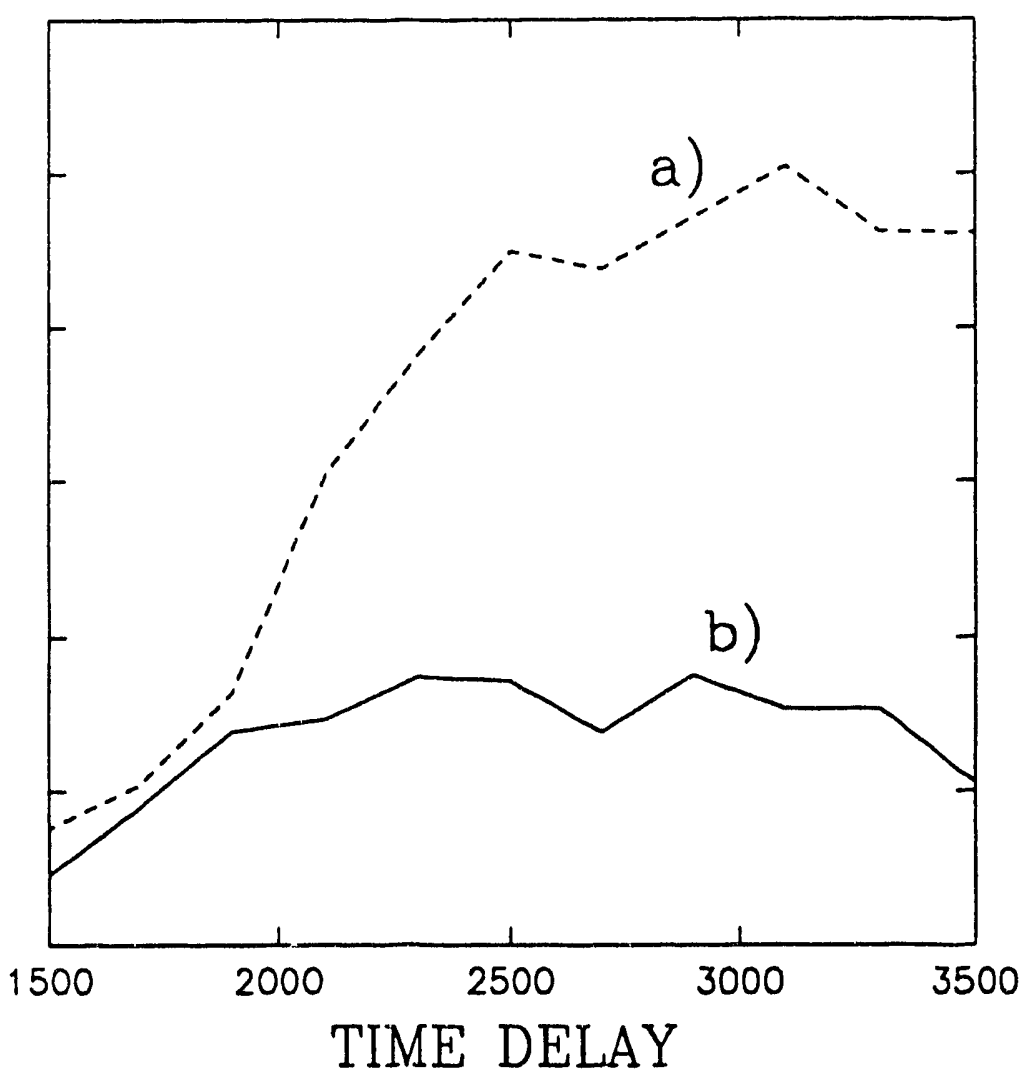

Figure 11 Experimental observation of $\mathrm{DH}(\mathrm{j}=7)$ signal which depended on the operation of both the photolysis and REMPI lasers, and both the DI and $\mathrm{H}_{2}$ pulsed valve, shown as a function of the time delay between the photolysis and REMPI lasers. Curve a) shows the DH signal observed with the $\mathrm{H}_{2}$ beam on, and curve b) shows the DH signal without the $\mathrm{H}_{2}$ beam. The signal was obtained by integrating the total signal in a section of the position-sensitive ion detector where the $\mathrm{H}_{2}$ beamdependent signal did not overlap with the DH background signal from the $\mathrm{D}$ atom beam. The $\mathrm{H}_{2}$ beam was operated at a pressure of $25 \mathrm{psig}$, and was triggered $400 \mu$ seconds prior to the generation of the $\mathrm{D}$ atom beam. The DI pulsed valve was operated at approximately 100 Torr and was mounted $1 \mathrm{~mm}$ below the reaction plane. 
Our main emphasis after the initial observations shown in Figure 11, has been on improving our understanding of the characteristics of the $\mathrm{D}$ and $\mathrm{H}_{2}$ beams, and on attempting to reduce the contamination of $\mathrm{DH}$ in the $\mathrm{D}$ atom beam, as described in the previous sections. We currently feel that a reasonably good understanding of the D and $\mathrm{H}_{2}$ beams has been obtained. Our measurements on the $\mathrm{D}$ atom beam production have provided us with a seemingly consistent picture of the dependence of the $D$ atom beam intensity on the conditions in the DI gas pulse, and leave us fairly confident that we are producing the $\mathrm{D}$ atom beam, which we are seeking. The same can be said for the $\mathrm{H}_{2}$ beam, which clearly achieves the required intensities. As far as the molecular beams are concerned, therefore, we feel confident that the required amounts of DH are being produced which, with the detection efficiency reported in chapter 2 , enable a successfull experiment.

More work may be necessary to deal with a few sources of noise in the experiment. As discussed in the previous section, some improvements have been observed in the reduction of background DH molecules, but efforts will certainly continue to improve this situation further. The effects of continued baking on the $\mathrm{DH}$ contamination in the $\mathrm{D}$ atom beam need to be studied, as well as the influence of improving the D atom beam collimation on the 'back-scattered' DH signal, which appears at long time delays between the photolysis and REMPI lasers. Recently some noise counts have been observed when very intense $\mathrm{H}_{2}$ beams were used, due to electron impact ionization, where the electrons are produced by scattered light from the mesh separating the extraction region from the flighttube. We have seen evidence that this noise signal is greatly reduced when the $\mathrm{H}_{2}$ beam collimation is improved, but perhaps improvement of the light baffles for the REMPI lasers is called for as well. All of the noise signals mentioned above are relatively small, most of them significantly less than one count per lasershot, but removal of these noise sources will greatly help in doing a succesfull experiment. 
Appendix A

The $\mathrm{I} / \mathrm{I}^{*}$ ratio in the photodissociation dynamics of DI at $205 \mathrm{~nm}$.

In the photodissociation of DI, the dissociation takes place on one of three potential energy surfaces, two of which correlate with the formation of ground state $I\left({ }^{2} \mathrm{P}_{3 / 2}\right)$, and which are reached by a perpendicular transition, and the other one correlating with the formation of $I^{*}\left({ }^{2} P_{1 / 2}\right)$, which is reached by a parallel transition. These two spin-orbit states of the I atom are separated by $0.94 \mathrm{eV}$. A measurement of the $\mathrm{D}$ atom velocity distribution, displaying a fast component correlating with the formation of $I$ and a slow component correlating with the formation of $\mathrm{I}^{*}$, allows for a determination of the $\mathbf{V}^{*}$ branching ratio and using a time-of-flight mass spectrometer Clear et.al. ${ }^{1)}$ determined the branching ratio for $\mathrm{HI}$ and $\mathrm{DI}$ photodissociation at $266 \mathrm{~nm}$, while Van Veen et.al. ${ }^{4}$ determined the branching ratio for $\mathrm{HI}$ photodissociation at 193, 222 and $248 \mathrm{~nm}$.

Our imaging detector allows for a straightforward determination of the $I / I^{*}$ ratio at $205 \mathrm{~nm}$, the wavelength which we employ for the two-photon resonant $3 \mathrm{~s}<--1 \mathrm{~s}$ excitation of $D$ atoms. If we employ non-Dopplerfree detection of the $D$ atoms resulting from the photodissociation, i.e. with a single laser beam, the REMPI laser selects an atomic velocity component $v_{x}$ in the plane of the detector along the laser axis. Likewise, on the images recorded, the ions show a displacement from the laser axis, which correlates with a velocity component $v_{y}$ in the plane of the detector perpendicular to the laser axis. Our detection scheme does not allow for a measurement of the third velocity component $v_{2}$, perpendicular to the plane of the detector, but in the case of DI photolysis, where the total D atom velocity is limited to two values, a measurement of $v_{x}$ and $v_{y}$, and in particular, the image profile along the $y$-axis, enables us to obtain some interesting results.

The REMPI laser, which is responsible for both the photolysis and the ionization of the $\mathrm{D}$ atoms, has a vertical polarization, i.e. perpendicular to the plane of the detector. Therefore, fast $\mathrm{D}$ atoms produced following a perpendicular transition, 
will have a recoil velocity which is preferentially in the plane of the detector, whereas slow D atoms, produced following a parallel transition, will have a recoil velocity which is preferentially out of the plane of the detector. At a given laser frequency, the images recorded on the detector, show the projection of all $\mathrm{D}$ atoms with a constant velocity

$$
v_{y \bar{z}}-\sqrt{ }\left(v_{\text {total }}^{2}-v_{x}^{2}(\omega)\right)^{2}
$$

on the $y$-axis. For a homogeneous angular distribution of the $D$ atoms the profile measured along the $y$-axis would be given by

$$
h(y) \propto \frac{1}{\sin \theta}
$$

where $\theta$ is the angle between the $y$-axis and the atomic velocity component in the yzplane. For a perpendicular transition this function is multiplied by an angular factor $3 / 2 \cos ^{2} \theta$, and for a parallel transtion this function is multiplied by an angular factor 3 $\sin ^{2} \theta$. For DI photolysis at $205 \mathrm{~nm}$, the theoretical profiles along the $y$-axis as a function of the Doppler shift for both a perpendicular and a parallel transition are shown in Figure 12. As anticipated the profiles for $D$ atoms formed by a perpendicular transition peak near the maximum velocity component $v_{y}$ determined by the choice of $v_{x}$, whereas the profiles for $D$ atoms formed by a parallel transition peak near $v_{y}=0$.

An experimental image showing $D$ atoms detected with the laser tuned to linecenter is shown in Figure 13. In order to record this image, the DI pulsed valve, located inside the DI chamber, was triggered about $400 \mu \mathrm{sec}$ before the laser was fired, allowing for a modest build-up of the DI partial pressure in the main chamber. As is apparant from Figure 13, D atoms are formed along the entire laser axis in this experiment, however, the important information is in the profiles of the D-atom along the $y$-axis and to obtain these profiles it is not necessary to photolyze the DI molecules at a well-defined position. It is immediately apparant from Figure 13 that the DI photolyis is dominated by the perpendicular transition.

Experimental profiles for the DI photolysis obtained at a number of Doppler 


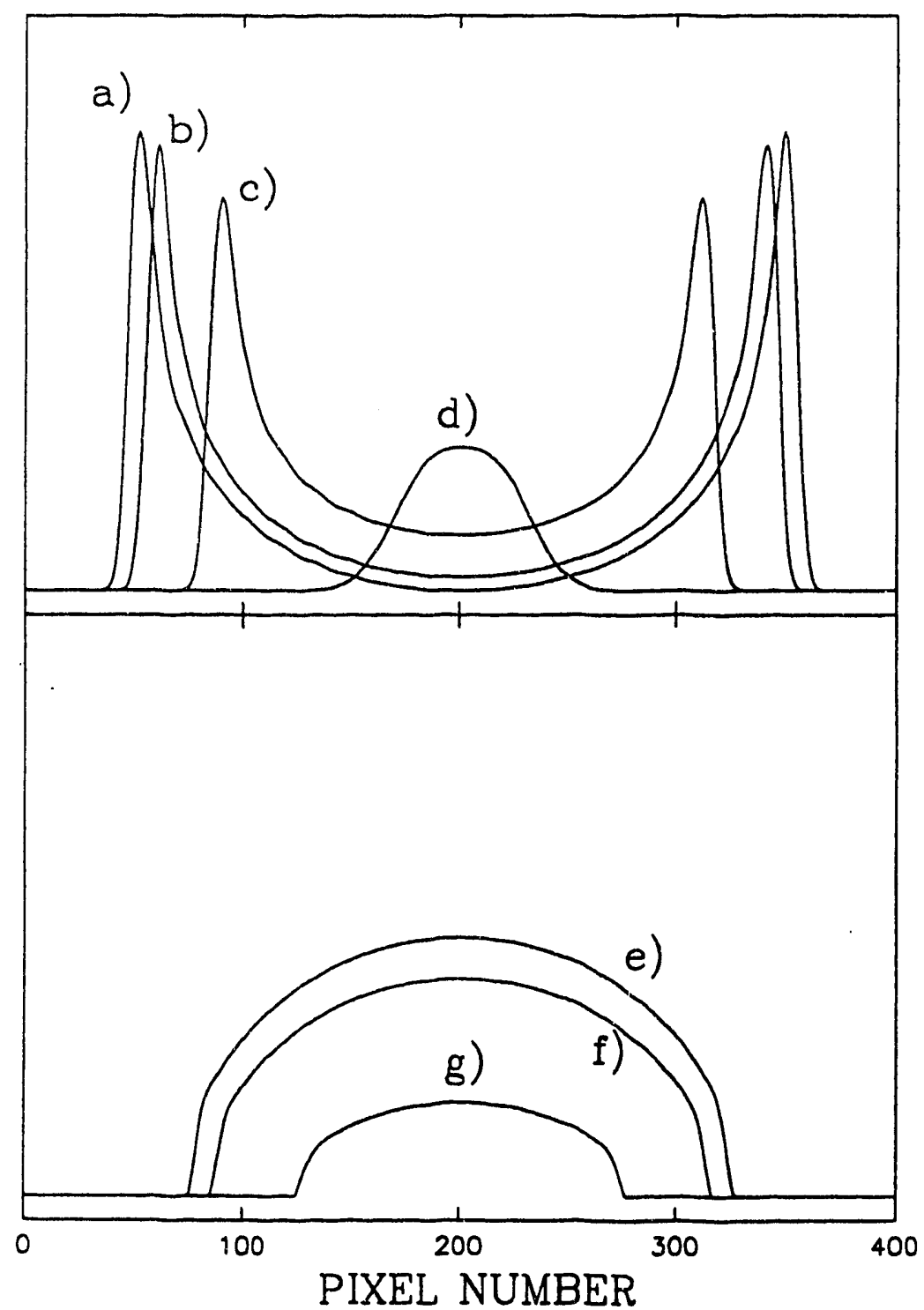

Figure 12 Theoretical $\mathrm{D}^{+}$image profiles, following photodissociation of $\mathrm{DI}$ at 205 $\mathrm{nm}$, as a function of the Doppler shift on the $3 \mathrm{~s}<--1$ transition of the $\mathrm{D}$ atom, for both a perpendicular and a parallel transition. Curves a) through d) show the theoretical curves for a perpendicular transition at Doppler shifts of $0 \mathrm{~cm}^{-1}, 1.8 \mathrm{~cm}^{-1}, 3.6 \mathrm{~cm}^{-1}$ and $5.4 \mathrm{~cm}^{-1}$, respectively, and curves e) through $\mathrm{g}$ ) show the theoretical curves for a parallel transition at Doppler shifts of $0 \mathrm{~cm}^{-1}, 1.8 \mathrm{~cm}^{-1}$ and $3.6 \mathrm{~cm}^{-1}$. 


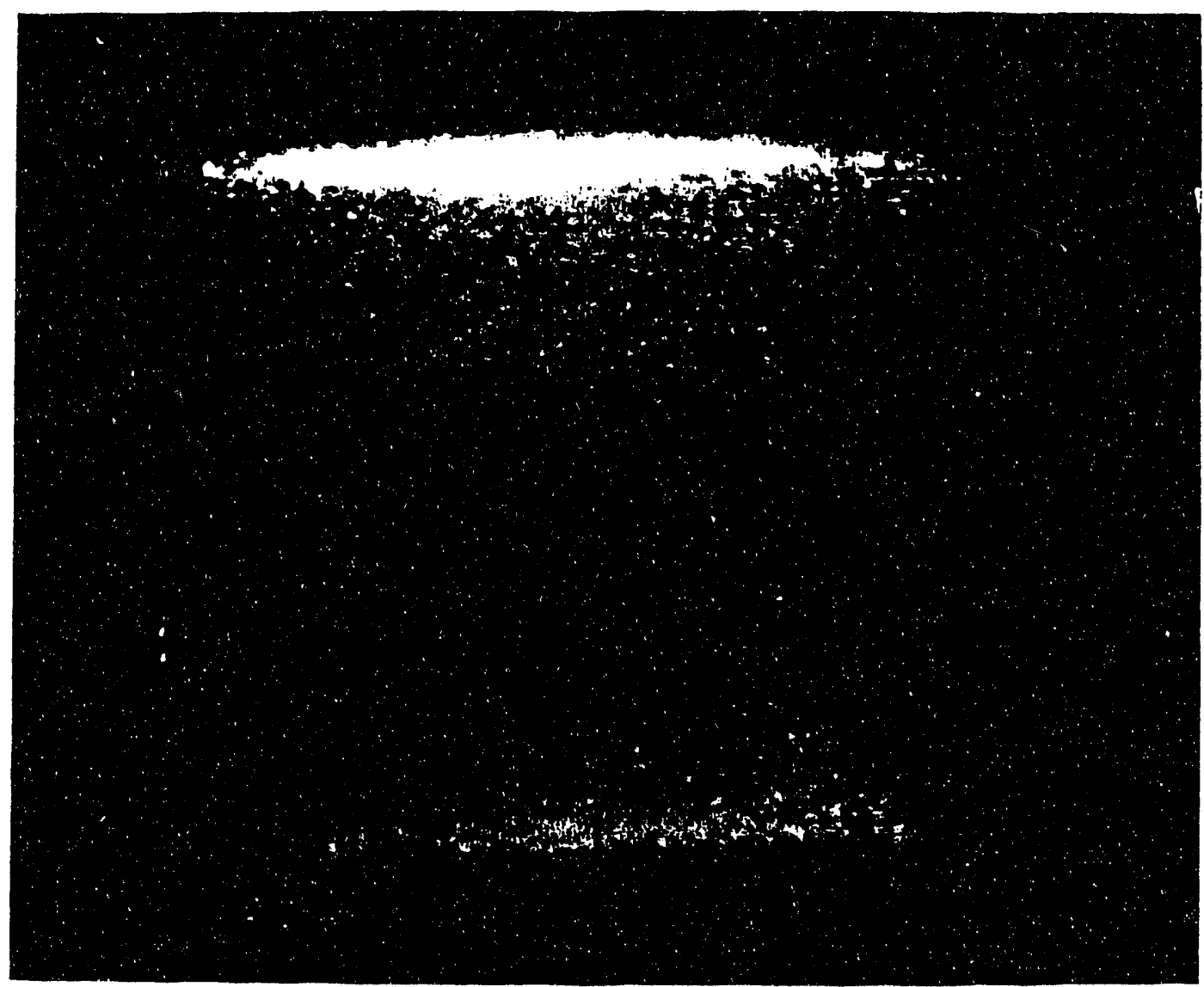

Figure 13 Experimental $\mathrm{D}^{+}$ion image, obtained for REMPI laser induced DI photolysis at $205 \mathrm{~nm}$, with the REMPI laser tuned to line-center of the $3 s$ r- 1 s transition of the D atom. 
shifts are shown in Figure 14. A fit of these profiles to a sum of contributions from the parallel and perpendicular transition revealed that the DI photolysis proceeds for $85 \pm 3 \%$ through the perpendicular transition and for $15 \pm 3 \%$ through the parallel transition. In these fits the only adjustable parameter was a parameter which serves as a resolution factor for the imaging detector and which also takes the thermal velocity of the DI precursor into account. The fits do not depend very sensitively on this parameter, which mostly determines the width of the peaks in the profiles for the DI photolysis.

It is interesting to compare these results to the calculations of Van Veen et.al.4) for the photodissociation of DI. At a wavelength of $205 \mathrm{~nm}$, they predict that $\mathrm{II}^{*}$ ratio should be approximately equal to 3 , which is significantly lower than our value of 5.7 \pm 1.4 . This information could be used to improve the potential energy curves for all the repulsive surfaces that play a role in the dissociation proces.

Since our DI sample contains a small impurity of $\mathrm{HI}$, it is also possible to study the photodissociation of $\mathrm{HI}$ at $205 \mathrm{~nm}$ with the technique described above. Initial results seem to suggest that for $\mathrm{HI}$ the role of the parallel transition is larger than for DI, but the HI data taken sofar are not very reliable due to the existence of a substantial $\mathrm{H}^{+}$background from the dissociation of background molecules in the chamber. At a point in the near future when the DI pulsed valve is being repassivated, the HI photolysis at $205 \mathrm{~nm}$ will be studied more carefully. 


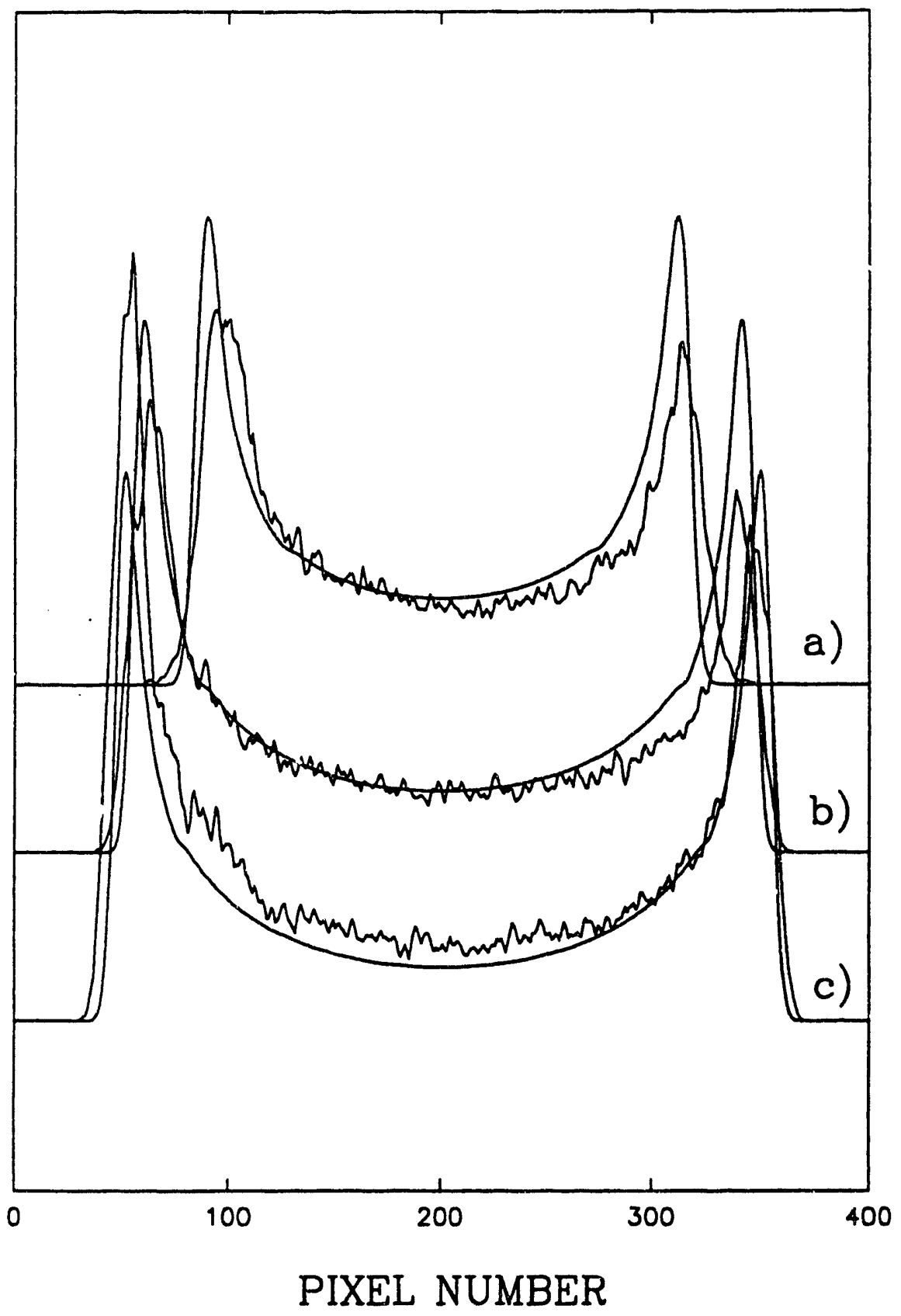

Figure 14 Experimental profiles for REMPI laser induced DI photolysis, at

Doppler shifts on the $3 \mathrm{~s}<--1 \mathrm{~s}$ D atom transition of a) $0 \mathrm{~cm}^{-1}$, b) 1.8 $\mathrm{cm}^{-1}$ and c) $3.6 \mathrm{~cm}^{-1}$, along with a fit assuming that the Dl photolysis takes place for $85 \%$ through a perpendicular transition and for $15 \%$ through a parallel transition. 


\section{References}

1) R.D. Clear, S.J. Riley and K.R. Wilson, J. Chem. Phys. $\underline{63}$, 1340 (1975).

2) R.E. Continetti, PhD thesis, University of California at Berkeley (1989).

3) W.R. Gentry and C.F. Giese, Rev. Sci. Instrum. 49, 595 (1978).

4) G.N.A. van Veen, K.A. Mohamed, T. Baller and A.E. de Vries, Chem. Phys. 80, 113 (1983). 

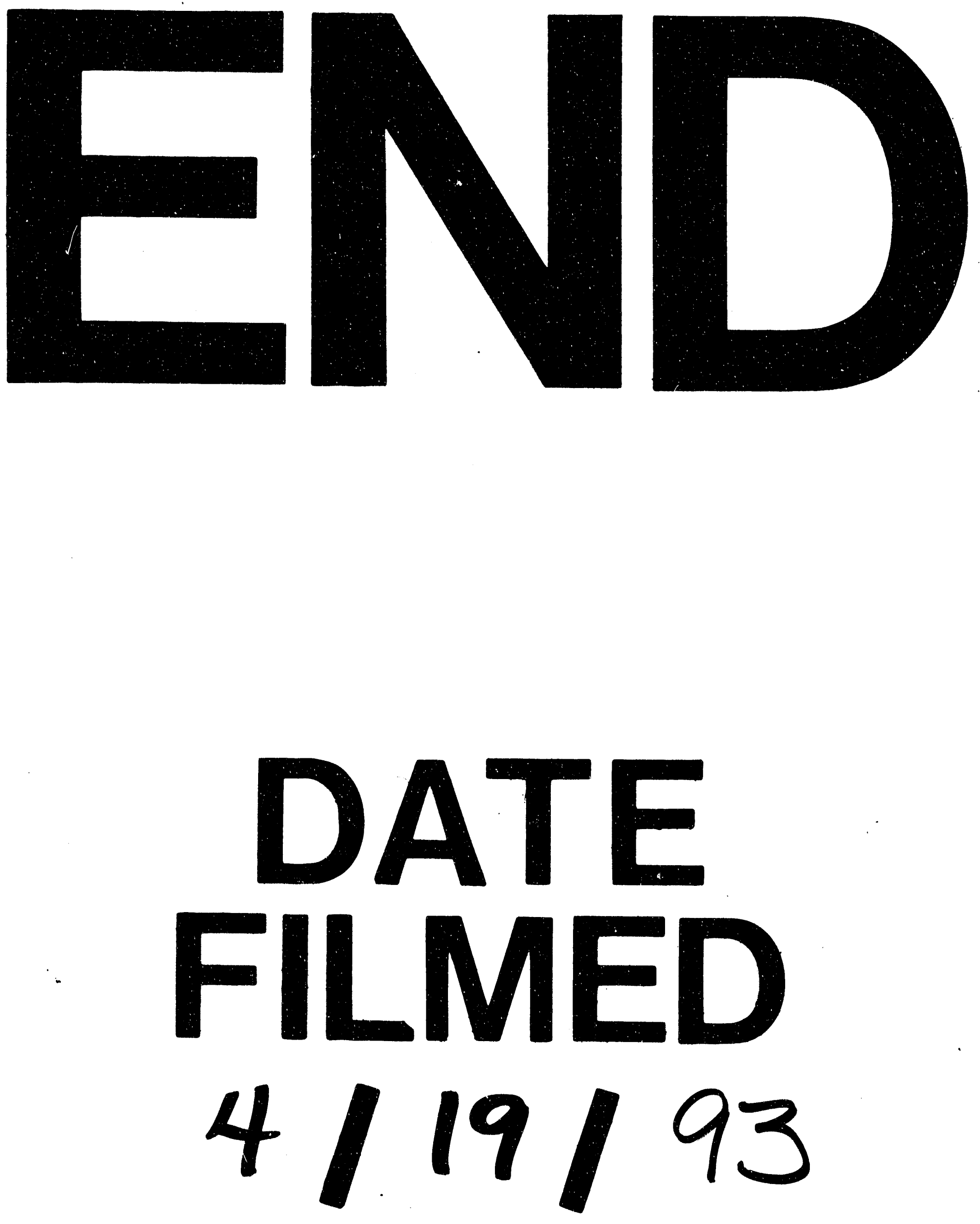

1 


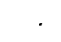

